\title{
CSHP Professional Practice Conference 2019: Poster Abstracts / Conférence sur la pratique professionnelle 2019 de la SCPH : Résumés des affiches
}

Facilitated Poster Sessions: Discussions of original research and pharmacy practice projects

Séance animée de présentations par affiches: Discussions sur des projets de recherche originale et des projets dans le domaine de la pratique pharmaceutique

\section{Sunday, February 3, 2019 • Dimanche 3 février 2019}

Infectious Diseases / Antimicrobial Stewardship

1. Improving Management of Clostridium difficile Infection by Direct Notification to Pharmacists

2. Development and Evaluation of an Infectious Diseases Education Program for Pharmacists

3. Sustaining Antimicrobial Stewardship Program in an ICU over More than 5 Years: Enabling ICU Pharmacy Practice

4. Determination of Antibiotic Susceptibilities in Aerococcus urinae Urinary Isolates

5. An Evaluation of Daptomycin Prescribing and Clinical Outcomes: A Retrospective, Single-Centre Experience

\section{Precepting / Teaching}

1. Determining Key Quality Assurance Indicators for Advanced Pharmacy Practice Experiences Placement Site Visits

2. Preceptor Experiences with Novel Student-Preceptor Models in Pharmacy Education: A Qualitative Analysis

3. Development of a Baseline Assessment Tool for Pharmaceutical Care Knowledge and Skills for Incoming Pharmacy Residents

4. Virtual Patients: Bridging the Gap between the Classroom and Clinical Pharmacy Practice

5. Atlantic Canadian Hospital Pharmacists in Direct Patient Care: Experiences as Preceptors

Medication Safety / Adverse Drug Events

1. Real-Time Tracking of Nursing Requests for Missing Medications

2. Kratom-Induced Acute Liver Failure

3. Autoimmune Hepatitis after Receiving One Cycle of Nivolumab/ Ipilimumab

4. Characterization of Medication-Related Near Miss Safety Events

5. Incidents Associated with Centralized Automated Processing of MultiMedication Compliance Packs

Drug Stability and Sterility

1. Chemical Stability of Cloxacillin in Sterile Water for Injection (SWFI) Stored in Polypropylene (PP) Syringes $(50$ and $100 \mathrm{mg} / \mathrm{mL})$ and Glass Vials $(250$ $\mathrm{mg} / \mathrm{mL}$ ) at $4^{\circ} \mathrm{C}$ and $25^{\circ} \mathrm{C}$

2. Stability of 1.0, 0.2 and $0.025 \mathrm{mg} / \mathrm{mL}$ Milrinone Solutions Stored in Syringes at $4^{\circ} \mathrm{C}$ and at Room Temperature $\left(25^{\circ} \mathrm{C}\right)$

3. Stability of Magnesium Sulphate Solutions in PVC Minibags, Non-PVC Minibags, and Polypropylene Syringes Stored at $4^{\circ} \mathrm{C}$

4. Stability of Cardioplegia Additive Solution in Polyvinyl Chloride Bags Stored at $4^{\circ} \mathrm{C}$ for 9 Days

5. Review of Microbial Contamination of Vials Used for Compounding with Closed System Drug Transfer Devices

\section{Clinical Pharmacy Practice}

1. Estimating the Proportion of Emergency Department Visits That Can Be Managed by Pharmacists' Expanding Scope in Ontario between 2010 and 2017

2. The Impact of Pharmacist Medication Management on 30-Day Hospital Re-Admission Rates as a Member of a Rapid Response Transitional Team

3. Do Knowledge Gaps about Opioids Exist for Pharmacists? A Closer Look at a Novel Online Learning Platform

4. Pharmacist Medication Reviews via Videoconference: A Prospective Cohort Study Pilot Study in Remote and Rural Underserviced Communities

5. Implementation of Electronic Special Access Program Forms to Improve Workflow for Pharmacists
Pharmacy Administration / Drug Distribution

1. Use of Extensive Auditing to Reduce Potential Diversion of Narcotics and Controlled Drugs in a Healthcare Facility

2. An Analysis of Medication Returns to Inpatient Pharmacy Using a ClosedLoop Health Information System

3. Utilization of the Electronic Health Record to Minimize Pharmacy Alert Fatigue

4. An Assessment of the Cost-Effectiveness of 24/7 Hospital Pharmacy

5. Identification and Selection of Preferred Candidates for the Position of Chief Executive Officer of a Professional Association

Pharmacotherapeutics

1. Comparison of Preventive Cardiovascular Pharmacotherapy in Surgical versus Percutaneous Coronary Revascularization

2. The Utilization of Mineralocorticoid Receptor Antagonists in Patients with Post ST-Elevation Myocardial Infarction Complicated by Left Ventricular Dysfunction

3. An Assessment of Modifiable Risk Factor Management in Hospitalized Patients with Type 2 Diabetes Mellitus

4. The Antithrombotic Treatment of Patients with Atrial Fibrillation Undergoing Percutaneous Coronary Intervention

5. A Retrospective, Observational Study of the Management of Patients Hospitalized with Heart Failure with Reduced Ejection Fraction

\section{Monday, February 4, $2019 \bullet$ Lundi 4 février 2019}

Infectious Diseases / Antimicrobial Stewardship

1. Evaluation of a Pharmacist-Led Antimicrobial Stewardship Service in a Pediatric Emergency Department

2. Evaluating the Impact of Prospective Audit and Feedback on the Use of Clindamycin and Quinolones in Clinical Teaching Units

3. A Retrospective Analysis of the Management of Staphylococcus aureus Bacteremia

4. Process Measures Associated with a Successful Antimicrobial Stewardship Intervention to Stop a Clostridium difficile Outbreak

Medication Safety / Adverse Drug Events

1. Severe Allergic Reaction Induced by Dexlansoprazole: A Case Report and Literature Review

2. Environmental Contamination with Nine Antineoplastic Drugs in 79 Canadian Centers

3. Adverse Effects of High-Dose vs Standard-Dose Dexmedetomidine in the Cardiac-Surgery Population: A Retrospective Cohort Study

4. Capturing Medication Safety Culture in Saskatchewan Pharmacies Using the Medication Safety Culture Indicator Matrix

5. Analyse descriptive des incidents et accidents médicamenteux de 2011 à 2018 dans un centre hospitalier

\section{Clinical Pharmacy}

1. Comparative Evaluation of Intentional versus Unintentional Medication Discrepancies during Admission Medication Reconciliation

2. Transition of an Independent Website for a Professional Association Branch to a Microsite Integrated within the Website of the Parent Organization

3. Opioid Stewardship: Implementing Proactive, Pharmacist-Led Reviews for Patients Co-Prescribed Opioids and Benzodiazepines at an Urban Academic Family Health Team

4. Optimizing Patient Education of Oncology Medications: A Quantitative Analysis of the Patient Perspective

5. Development of Candidate Choosing Wisely Recommendations for a Professional Society 
Pharmacy Administration / Drug Distribution

1. The Impact of Delisting Docusate from a Hospital Formulary

2. Pharmacist Review of Computer Physician Medication Order Entry in Hospitals: A Prospective Observational Study of Pharmacist Interventions

3. Determining Patient and Caregiver Values and Needs from an Outpatient Oral Anticancer Therapy Program: A Qualitative Needs Assessment

4. Telepharmacist Medication Order Review: A Prospective Observational Study in Healthcare Systems

5. Patients' Perspectives on a Self-Administration of Medication Program in a Rehabilitation Hospital

\section{Tuesday, February 5, 2019 • Mardi 5 février 2019}

Infectious Diseases / Antimicrobial Stewardship (1)

1. Clinical Burden of Antibiotic Resistance Following Implementation of a Multidisciplinary Antimicrobial Stewardship Initiative in a Major Tertiary Care Center: A Controlled Interrupted Time Series Analysis over 14 Years

2. Ceftobiprole plus Vancomycin for the Treatment of Methicillin-Resistant Staphylococcus Aureus Infective Endocarditis: Case Report

3. Antimicrobial Guideline Concordance is Low in Cancer Patients with Febrile Neutropenia Admitted to General Internal Medicine at an Academic Hospital

4. Drug Utilization Evaluation of Ceftolozane/Tazobactam in a Canadian Academic Teaching Hospital System

5. A Retrospective Case Series Assessing Ceftolozane/Tazobactam Use at a Large Academic Centre

\section{Infectious Diseases / Antimicrobial Stewardship (2)}

1. Using Monte Carlo Simulation to Evaluate Tigecycline Dosing Strategies for Bacteria with Raised Minimum Inhibitory Concentrations in NonCritically Ill Patients

2. Implementation and Suspension of an Antimicrobial Stewardship Audit and Feedback Program: Impact on Antimicrobial Utilization Patterns in an Inpatient General Internal Medicine Unit

3. Voriconazole Prophylaxis in Leukemic Patients: A Retrospective Single Centre Study (award-see page 62)

4. Assessment of a Therapeutic Drug Monitoring Strategy of Once Daily Dosing of Gentamicin/Tobramycin in Paediatric Patients

5. A Drug Use Evaluation of Aerosolized Ribavirin at a Canadian Teaching Hospital

Medication Safety / Adverse Drug Events (1)

1. Hypoglycemia in Paediatric Cardiology Patients Initiated on Propranolol: A Retrospective Review

2. Anticholinergic Potential Risk Assessment Scales: Comparison of Drugs and Risk Scores

3. Photosensitivity Associated with Long-Term Voriconazole Therapy: A Case Report

4. Safety of Enteral Nutrition Interruption around Levothyroxine Doses in Critical Ill Patients

5. Étude rétrospective des accidents et incidents associés à la documentation des doses de médicaments dans un hôpital universitaire

Medication Safety / Adverse Drug Events (2)

1. Rapid Onset of Cholelithiasis in an Adult Treated with Ceftriaxone

2. Evaluation of the Use of 'Do Not Use Abbreviations' in Hospital Orders: A Quality Assurance Audit

3. Leveraging the Electronic Health Record for Medication Safety Indicators

4. Implementation of Barcode Medication Administration Using a Quality Improvement Approach

5. Factors Influencing Prescribing of Direct Oral Anticoagulants in the Elderly Leading to Adverse Outcomes: An Analysis from the Windsor Region
Clinical Pharmacy (1)

1. Medication Reconciliation at Hospital Admission: Proactive versus Retroactive Models

2. Assessing Medication Reconciliation in Hospitalized Adult Patients Discharged from Accountable Care Units in Saskatchewan Health Authority - Regina

3. The Utilization of Splenectomy Post-Op Clinical Vaccinations Order Sets to Enhance Adherence and Timeliness of Vaccinations in Splenectomy Patients: A Pre-and-Post Intervention Study

4. Utilization of Health Literacy Assessment Tools to Tailor Patient Counselling

5. Development of an Inpatient Pharmacist Diabetes Educator Role

Clinical Pharmacy (2)

1. Analyse descriptive des publications dans le domaine de la pharmacie 1973 à 2016

2. Face and Content Validation of an Instrument to Measure Medication Management Capacity in Older Adults

3. Improving Pharmacist-Pharmacist Communication at Hospital Discharge

4. Exploring the Perspectives of Healthcare Professionals in Delivering Optimal Oncology Medication Education

5. Development and Implementation of a Competency Assessment Tool for Hospital Pharmacists

Pharmacotherapeutics

1. Transition to Insulin Pens in Inpatient Rehabilitation and Mental Health Care Hospitals

2. Point Prevalence Survey of Benzodiazepine and Sedative-Hypnotic Drug Use in Long-Term Care

3. Benzodiazepine and Sedative-Hypnotic Drug Use in Hospital: Perspectives from Healthcare Providers

4. Investigation of the Average Duration of Dual Antiplatelet Therapy in Dialysis and Pre-Dialysis Patients

5. Revue de l'utilisation de l'eculizumab

Drug Stability and Sterility (1)

1. Preliminary Evaluation of the NAPRA 6-hour Rule for Single-Use Vials after First Puncture in an ISO-5 Environment

2. Physical Compatibility and Stability of Ascorbic Acid Injection in Polyvinyl Chloride Minibags at $4{ }^{\circ} \mathrm{C}$ and Room Temperature $\left(25^{\circ} \mathrm{C}\right)$

3. Stability of $2.5 \mathrm{mg} / \mathrm{mL}$ Indocyanine Green (ICG) Solutions Stored in Syringes at $25^{\circ} \mathrm{C}, 4^{\circ} \mathrm{C},-20^{\circ} \mathrm{C}$ and $-67^{\circ} \mathrm{C}$

4. Development of an On-Going Sterility Monitoring Program for Single-Use Vials Undergoing Multiple Access Following Application of a Closed System Transfer Device

Drug Stability and Sterility (2)

1. Stability of $0.04,0.1$, and $0.2 \mathrm{mg} / \mathrm{mL}$ Vitamin $\mathrm{K}$ (Phytonadione) in $5 \%$ Dextrose in Water Solutions Stored in Polyvinyl Chloride Bags at $4^{\circ} \mathrm{C}$ over 9 Days

2. Élaboration d'outils et de politiques et procédures sur les préparations stériles

3. Stability of $9.1,10.7$ and $16.7 \mathrm{mg} / \mathrm{mL}$ Calcium Gluconate Solutions in Normal Saline and/or 5\% Dextrose in Water Stored in Polyvinyl Chloride Minibags at $4^{\circ} \mathrm{C}$ over 9 Days 


\section{Improving Management of Clostridium difficile Infection by Direct Notification to Pharmacists}

\author{
Dhami R $R^{1,2}$, Popovski $Z^{1}$, Elsayed $S^{1,3}$ \\ ${ }^{1}$ London Health Sciences Centre, London, ON \\ ${ }^{2}$ University of Waterloo, Waterloo, ON \\ ${ }^{3}$ Western University, London, ON
}

Background: Infection Prevention and Control (IPAC) conducted a multidisciplinary, retrospective review of Clostridium difficile infection (CDI) cases to identify process improvement opportunities in the prevention and treatment of CDI. In addition to important infection control interventions, the pharmacist's scope of practice was recognized to be valuable in identifying medication-related issues in the treatment of CDI.

Description: The goal of this collaborative project between Microbiology and Antimicrobial Stewardship was to reduce rates of CDI, improve treatment of CDI by increasing adherence to guidelines, and by involving the pharmacist in each CDI case.

Methods: A novel process was developed whereby the Microbiology Lab provided direct email notification of a positive CDI result to the pharmacist team. Upon receipt, the most responsible pharmacist for that patient assessed the severity of CDI, suggested initial therapy and addressed medication-related risk factors. Each case was documented electronically and interventions were tracked. Descriptive statistics were used for analysis.

Evaluation: Over the 1 year study period, CDI rates decreased, and no CDI outbreaks had occurred. Pharmacists had intervened on $>90 \%$ of cases. Of the assessments completed, an average of $25 \%$ of cases required intervention on the initial drug therapy regimen and an average of $36 \%$ of patients required risk factor modification. Time from notification to first dose of antibiotic was reduced to $<2$ hours from an average of 3.4 hours. The acceptance rate for interventions was $>90 \%$ on initial therapy and $80 \%$ for risk factor modification.

Implications: Overall, this process has demonstrated the need and value for proactive pharmacist involvement in CDI treatment and a pharmacist review of all CDI cases has now become standard of care at this centre.

\section{Development and Evaluation of an Infectious Diseases Education Program for Pharmacists}

\author{
Haj $R^{1}$, Leung $E^{1,2}$, Dewhurst $N F^{1,2}$, Tom $E^{1}$, Halapy $H^{1,2}$, Chan $S C^{2}$, \\ Chang $\mathrm{Cf}^{2}$, Chant $\mathrm{C}^{1,2}$ \\ ${ }^{1}$ St. Michael's Hospital, Toronto, ON \\ ${ }^{2}$ Leslie Dan Faculty of Pharmacy, University of Toronto, Toronto, ON
}

Background: As part of a broader practice standardization project, an education program was developed focussing on a series of high risk, but commonly seen therapeutic topics. Infectious disease management is a complex area of study that requires pharmacists to have minimal baseline knowledge in order to identify and resolve related drug therapy problems (DTPs).

Description: To ensure pharmacists possess the required knowledge and skills, an infectious diseases education module was developed, implemented and evaluated.

Action: The infectious diseases education module consisted of a voicedover slideshow presentation, which included supporting institutional policies and procedures. The education module underwent review and feedback from expert and typical pharmacist users prior to deployment. Assessment of the pharmacist's knowledge and skills consisted of a 21-question multiple choice test that was administered both at baseline and after review of the module. Point-biserial (p-bis) and p-values were used to ensure test question validity and reliability. Pharmacists were required to score at least $80 \%$ on the post-module test. Program evaluation is consisted of a questionnaire asking about the pharmacist's own confidence and of their colleagues to identify and resolve infectious diseases related DTPs, and the perceived value of the program.

Evaluation: Fifty-two pharmacists completed the pre- and post-module tests. Post-module completion, the average test score increased from $85 \%$ to $94 \%$. The majority of the pharmacists $(51 / 52$ [98\%]) passed the test. Responses from the post-module questionnaire indicated that pharmacists were overall confident in their own and colleagues' ability to identify and resolve infectious disease DTPs, and perceived the program as beneficial to improve patient care and safety.

Implications: The results suggest that pharmacists benefitted from an infectious diseases education program. Completion of the education module and post-module test are now mandatory for all new staff. Based on the program's success, future modules on different therapeutic topics are in development.

\section{Sustaining Antimicrobial Stewardship Program in an ICU over More than 5 Years: Enabling ICU Pharmacist Practice}

Ho V, Ross I, Brooks A, Mertz D, Karachi T, Wynne C

Hamilton Health Sciences, Juravinski Hospital and Cancer Centre, Hamilton, $O N$

Background: Antimicrobial agents are one of the most prescribed medications in the intensive care unit (ICU). The goal of an antimicrobial stewardship program (ASP) is to promote appropriate antimicrobial use to optimize patient outcomes. We aimed to evaluate the impact of two ICU pharmacists in promoting stewardship practices and changing antimicrobial prescribing during daily multidisciplinary rounds in a 20-bed medical-surgical ICU.

Description: We tracked the number and type of interventions by ICU pharmacists and ASP team. Outcomes include defined daily doses (DDD), length of ICU stay and mortality.

Action: Formalized ASP audit and feedback rounds involving infectious diseases and ICU physicians and pharmacists started in 2011, initially weekly then bi-weekly. ICU pharmacists took on the responsibility for promoting and documenting ASP activities on daily multidisciplinary ICU rounds.

Evaluation: Since 2013, ICU pharmacists documented 3548 interventions and ASP team documented 296 interventions. ICU pharmacists made $92 \%$ of all interventions. The majority of interventions were accepted by the intensivists ( $90 \%$ of ICU pharmacist and $99 \%$ of ASP team interventions). ICU pharmacist interventions have decreased from 3 to 2.6 per day. Similarly, ASP team interventions have decreased from 1.4 to 1.27 per ASP rounds. Types of ICU pharmacist interventions include dosing (43\%), spectra (15\%), discontinuation (15\%) and duration (15\%). Prescribing of broad spectum antibiotics and antifungals has decreased since 2013: DDD $(-32 \%)$, carbapenems $(+35 \%)$, vancomycin $(-23 \%)$, floroquinolones $(-60 \%)$, piperacillin-tazobactam $(-19 \%)$ and antifungals $(-47 \%)$. Average mortality for patients admitted to ICU for more than 72 hours was $9 \%$. Average length of ICU stay was 5 days. Both were similar to prior ASP implementation.

Implications: Given their daily presence, ICU pharmacists can make more ASP interventions than ASP teams who are not present daily. ICU pharmacists complement a successful and sustainable ASP as shown by the reduction in antimicrobial utilization and changes in prescribing culture. 


\section{Determination of Antibiotic Susceptibilities in Aerococcus urinae Urinary Isolates}

\section{Krishnan A, Nadeau L}

Windsor Regional Hospital, Windsor, ON

Background: Aerococcus urinae is a Gram-positive organism, initially identified in 1992. It is known to cause urinary tract infections (UTIs), bacteremia, and endocarditis in humans. There is limited data regarding the susceptibility of $A$. urinae to first-line antimicrobials indicated for the treatment of UTIs. In 2016, $A$. urinae was isolated in 125 urine samples processed by four hospitals in the region. The unfamiliarity with this organism and the lack of local antimicrobial susceptibility rates presents a challenge for clinicians and often results in the unnecessary use of broadspectrum antibiotics.

Objectives: The primary objective of this study was to establish the susceptibility rate of $A$. urinae urinary isolates to cefazolin, ampicillin, nitrofurantoin, fosfomycin, and ciprofloxacin. The secondary objective was to identify demographic characteristics associated with $A$. urinae bacteriuria in our patient population.

Methods: Urinary samples received by the laboratory from October 2017 to June 2018 underwent routine identification as per physician orders. Samples that grew $A$. urinae were included in this study and subjected to susceptibility testing. Susceptibility testing was conducted via disk diffusion and results were interpreted based on published breakpoints for zone diameters. Results were analyzed using descriptive statistics.

Results: A total of 72 isolates were included. Susceptibility rates for cefazolin, ampicillin, nitrofurantoin, fosfomycin, and ciprofloxacin were $100 \%, 99 \%, 99 \%, 96 \%$, and $65 \%$, respectively. The average age of patients was 79 years, $63.9 \%$ were female, $31.9 \%$ were recently hospitalized, and $44.4 \%$ were residents of a long-term care facility.

Conclusion: Cefazolin, ampicillin, nitrofurantoin, and fosfomycin demonstrated good in vitro activity against $A$. urinae. In contrast, ciprofloxacin demonstrated decreased activity against this organism. Currently recommended first-line agents for the management of uncomplicated UTIs could be utilized to treat this organism. Characteristics of patients with $A$. urinae bacteriuria are consistent with risk factors predisposing to UTIs.

\section{An Evaluation of Daptomycin Prescribing and Clinical Outcomes: A Retrospective, Single-Centre Experience}

Tsoi VK ${ }^{1}$, Bombassaro $A M^{1,2}$, Elsayed $S^{1,2}$, Facca $N^{1}$, Dhami $R^{1,2,3}$

${ }^{1}$ London Health Sciences Centre, London, ON

${ }^{2}$ Western University, London, $O N$

${ }^{3}$ University of Waterloo, Waterloo, $\mathrm{ON}$

Background: Daptomycin is approved for skin/soft tissue infections due to $S$. aureus and selected Streptococcus spp., and S. aureus bacteremia/ endocarditis (right-sided, native valve). Prescribing has increased at our institution since 2015.

Objectives: The study objectives were to describe prescribing patterns of daptomycin, and to identify the rate and predictors of clinical failure in the overall population and vancomycin-resistant Enterococcus (VRE) subgroup.

Methods: This was a retrospective cohort study of adults prescribed daptomycin for $>48$ hours (without exposure in the preceding 3 months) between April 2015 and March 2017. Primary outcomes were patient, infection, and treatment factors associated with daptomycin prescribing. Secondary outcomes were the rate and predictors of clinical failure (a composite of in-hospital mortality, daptomycin discontinuation due to toxicity or suboptimal response, and readmission for or retreatment of the index infection). Descriptive and multivariate analyses were performed.

Results: Among 81 patients enrolled, $58.0 \%$ were male, median age was 60 years, and $42.0 \%$ had a Charlson Comorbidity Index of $\geq 5$. Of 38 bacteremic patients, $18.4 \%$ had a Pitt Bacteremia Score of $\geq 4$ and $52.6 \%$ were bacteremic for $>4$ days. The most common infections were bloodstream, intraabdominal, and bone/joint. VRE was isolated in $50.6 \%$ of patients. Prescribing was off-label in $88.9 \%$ of cases. The median dose was $6 \mathrm{mg} / \mathrm{kg}$ overall and in the VRE subgroup. The clinical failure rate was $37.0 \%(30 / 81)$ overall with 13 deaths, and $46.3 \%(19 / 41)$ in the VRE subgroup with 9 deaths. The risk of clinical failure increased with bacteremia for $>4$ days (OR 6.36, 95\% CI 1.34-30.17, $p=0.02$ ).

Conclusions: Daptomycin was mainly prescribed off-label for VRE. The median dose of $6 \mathrm{mg} / \mathrm{kg}$ was consistent with manufacturer recommendations for $S$. aureus bacteremia. The clinical failure rate in the VRE subgroup exceeded the overall rate, suggesting antimicrobial stewardship opportunities to evaluate formulary restrictions, indications, and dosing for daptomycin.

\section{Determining Key Quality Assurance Indicators for Advanced Pharmacy Practice Experiences Placement Site Visits}

Ho $C^{1,2}$, Li S $S^{2}$, Stankic $A^{2}$

${ }^{1}$ Institute for Safe Medication Practices Canada, Toronto, ON ${ }^{2}$ Leslie Dan Faculty of Pharmacy, University of Toronto, Toronto, ON

Background: In order to perform effective experiential education placement site visits, there should be a standard set of key quality assurance (QA) indicators.

Description: The objective of our study was to generate a consensus among experiential education faculty of Canadian pharmacy schools for the most important key QA indicators for Advanced Pharmacy Practice Experiences (APPE) site visits.

Action: We surveyed members of the Pharmacy Experiential Programs of Canada using an online two-round Delphi questionnaire, with a focus on four main categories of QA indicators for APPE site visits: (1) learning-centered environment; (2) preceptor-related factors; (3) studentrelated factors; and 4) organization of placement site.

Evaluation: We identified the top three indicators for each category. For category 1, the top-ranked indicators were: defined roles for students; student access to drug information and patient records; and adequate physical space for student work. For category 2, the top three were: regular and consistent feedback provided to students by preceptor; preceptor availability; and preceptor's professionalism. For category 3, student involvement in pharmaceutical care processes; student providing counselling to patients; and student involvement in expanded-scope practices were the highest ranked indicators. For category 4, clear learning objectives; specific examples used in the midpoint/final evaluations; and activities planned to meet learning objectives were the most important. Implications: Implementing an APPE site QA indicator checklist for the entry-to-practice Doctor of Pharmacy curriculum in Canadian institutions is of high priority as we develop and advance our experiential education programs for training new pharmacy professionals. 


\section{Preceptor Experiences with Novel Student- Preceptor Models in Pharmacy Education: A Qualitative Analysis

\author{
Cicinelli $E^{1,2}$, LeBlanc $K^{1,2}$, Cameron $K^{2}$, Fernandes $O^{1,2}$, McIntyre $C^{3}$, \\ Bjelajac Mejia $A^{2}$, Natsheh $C^{1,2}$ \\ ${ }^{1}$ University Health Network, Toronto, ON \\ ${ }^{2}$ Leslie Dan Faculty of Pharmacy, University of Toronto, Toronto, ON \\ ${ }^{3}$ Montfort Hospital, Ottawa, ON
}

Background: Implementation of the Entry-to-Practice Doctor of Pharmacy program in Canadian pharmacy education has required institutions to host more students for experiential rotations. In response, some institutions have explored novel student-preceptor models: peer-assisted-learning (PAL; $\geq 2$ students of the same educational level), near-peer-teaching (NPT; $\geq 1$ junior student(s) with $\geq 1$ senior student(s)), and co-preceptorship (CoP; $\geq 2$ preceptors).

Description: The objectives of this study were to describe the experiences of pharmacy preceptors in novel student-preceptor models and to assess the models using Kirkpatrick's framework for evaluating educational interventions.

Action: Pharmacists who hosted final-year pharmacy students in novel student-preceptor models in institutional settings were interviewed. Transcripts were coded and analyzed using Kirkpatrick's framework to generate themes about participants' experiences and perceptions of the models.

Evaluation: Twenty preceptors from 13 institutions were interviewed, and 13 themes were identified. Fourteen preceptors had experience with PAL, 9 with NPT, and 9 with CoP. Preceptors perceived that NPT and PAL fostered comfortable learning environments that supported students' success; challenges included increased time spent teaching multiple students and completing evaluations. CoP allowed preceptors to balance teaching with clinical duties while broadening students' exposure to different practice settings. Preceptors improved skills in time management, communicating feedback, and adapting to students' learning needs. Novel rotation models allowed preceptors to provide care to more patients and complete projects, thus extending their professional practice. They also perceived that students participating in these models developed a greater sense of responsibility for patient care, and they are primed to work collaboratively with pharmacy colleagues.

Implications: Preceptors expressed satisfaction with novel studentpreceptor models. The models enhanced the learning, skill development, and professional practice of both preceptors and students. Widespread adoption of these models in pharmacy experiential education would support students' development of knowledge, skills, attitudes, and behaviours essential for their future practice.

\section{Development of a Baseline Assessment Tool for Pharmaceutical Care Knowledge and Skills for Incoming Pharmacy Residents}

Ou $X^{1}$, Halapy $H^{1,2}$, Satchu $S^{1,2}$

${ }^{1}$ Leslie Dan Faculty of Pharmacy, University of Toronto, Toronto, ON ${ }^{2}$ St. Michael's Hospital, Toronto, ON

Background: Hospital residency programs utilize various tools to assess baseline knowledge and experiences of new residents. No baseline assessment tool has been systematically proven to predict the academic performance of residents or accurately reflect the impact of the residency program on resident learning.

Description: The authors developed a baseline assessment tool for incoming pharmacy residents to better assess baseline pharmaceutical care knowledge and skills.
Action: An external scan of existing tools was conducted with provincial pharmacy residency programs. A literature search for existing baseline assessments in academia was completed using key words (competencies, entry-level, baseline, evaluation, residency, needs assessment, background knowledge, student, pharmacy, medical, nursing) in scientific and educational search engines (Ovid MEDLINE, ProQuest, Web of Science, Scopus, Google Scholar). Thirty articles were retrieved, and 15 were deemed relevant through independent review by two authors. The article review was also complemented with a final discussion between all authors. Utilizing an iterative process, a baseline test was developed consisting of a pharmaceutical care-based case coupled with an assessment rubric. The rubric assesses 4 competency domains (problem recognition and work-up, organization of thinking, critical thinking and therapeutic knowledge and problem solving). Face and content validity were evaluated by expert content reviewers.

Evaluation: The tool was piloted on the incoming resident of 2018 as a 90-minute open-book test followed by a resident oral presentation and coordinator debrief guided by the rubric. Feedback from the pilot test suggested the test was too long, but otherwise provided a good framework for discussion about the resident's baseline pharmaceutical care knowledge and skills.

Implications: The tool provided useful feedback on the baseline pharmaceutical care knowledge and skills of the resident. More widespread use with systematic evaluation of the assessment tool is planned.

\section{Virtual Patients: Bridging the Gap between the Classroom and Clinical Pharmacy Practice}

Dahri $K^{1,2}$, Chan $F^{1}$, Seo $M J^{1}$, Seto $K^{1}$, Yeung $J^{1}$

${ }^{1}$ Faculty of Pharmaceutical Sciences, University of British Columbia,

Vancouver, $B C$

${ }^{2}$ Pharmacy Department, Vancouver General Hospital, Vancouver, BC

Background: When students encounter real patients during their experiential rotations they are overwhelmed with the quantity of information available limiting their ability to care for patients. Virtual patients (VPs) provide a bridge from classroom knowledge acquisition to real-life knowledge application. VPs give students the opportunity to engage in simulated clinical scenarios which includes navigation of a construct of medical records and patient interviews, in order to assess a patient's medication therapy. While benefits exist, the development and implementation of VPs is a time intensive process.

Objective: To evaluate the incorporation of VPs into an Entry to Practice Doctor of Pharmacy Program.

Methods: A 16-question survey was disseminated to all Year 1, 2 and 3 students. In addition, as Year 2 students were the first students to encounter the VPs, they were also invited to participate in a focus group. Descriptive statistics were used to analyze the survey data. The focus group was audio recorded, transcribed and thematically analyzed.

Results: A total of 180 students participated in the survey for a response rate of approximately $28 \%$. From all three classes, $170 / 180(94.4 \%)$ of respondents strongly agreed/agreed that the incorporation of VPs was valuable for their learning. Students felt the VP cases helped them to learn more about the main medical conditions in the case (YR1 $=72 \%$; YR2 $=87 \%$; YR3 $=90 \%$ ) and develop their clinical reasoning skills (YR1=78\%; YR2=97\%; YR3=95\%). Participants in focus groups described the following benefits to their learning: a) becoming active decision makers through engaging in a process-based experience; b) being exposed to, and gaining knowledge about, 'real world' cases and documents (e.g., nurses notes); and c) applying and solidifying classroombased learning. 
Conclusion: Students find VPs to be valuable to their learning and development of clinical reasoning skills.

\section{Atlantic Canadian Hospital Pharmacists in Direct Patient Care: Experiences as Preceptors}

Davies $H^{1}$, Doucette $D^{2}$, Whelan $A M^{1}$

${ }^{1}$ College of Pharmacy, Dalhousie University, Halifax, NS

${ }^{2}$ Horizon Health Network, Moncton, NB

Background: Expanded experiential learning is a major component of the Doctor of Pharmacy degree program being developed at Dalhousie University. Hospital practice sites and preceptors will be vital to clinical learning. A large survey of hospital pharmacy preceptors in the region affiliated with Dalhousie University was conducted to understand the experiences, learning needs, barriers, motivators, and interest in various preceptor models.

Description: The part of the project presented here describes precepting experiences of pharmacists in direct patient care.

Action: A literature search was conducted to identify examples of preceptor surveys. Items for the questionnaire came from the literature and the project's Advisory Board members. The draft was piloted, and changes made. In May 2017, the regional hospital pharmacy management group, the local branches of the Canadian Society of Hospital Pharmacists, Faculty at Dalhousie University, and representatives from hospitals in the region were emailed an invitation to participate in the on-line survey. Statistical analysis was completed using Minitab Statistical Software Version 14.

Evaluation: Approximately $57 \%$ of respondents indicated that they were pharmacists in direct patient care and of this group 53\% had worked 10 years or less with $40 \%$ being from New Brunswick and 37\% from Nova Scotia. Ninety-seven percent of the pharmacists in direct patient care had served as a preceptor, with $53 \%$ indicating that they had done so for 7 or more years. Fifty-three percent of pharmacists in direct patient care had precepted 1 or 2 undergraduate pharmacy students in the previous 12 months, with $83 \%$ reporting spending greater than 7 hours per week in precepting related activities. The two most common reasons for not serving as a preceptor were "competing priorities" and "insufficient time". Implications: This information helps inform the development of resources and strategies to sustain pharmacy clinical learning within Atlantic Canadian hospitals.

\section{Real-Time Tracking of Nursing Requests for Missing Medications}

Satchu $S^{1,2}$, Dewhurst $N^{1,2}$, Sutradhar $S^{2}$, Carroll $K^{1}$, Grant $D^{1}$, Bhathena $M^{1}$, Wong $B^{1}$, Chant $C^{1,2}$

${ }^{1}$ Leslie Dan Faculty of Pharmacy, University of Toronto, Toronto, ON ${ }^{2}$ St. Michael's Hospital, Toronto, ON

Background: Despite advances in medication systems technology, "missing medications" remain a considerable source of frustration, wasted time, effort and money in the medication distribution and administration process. Nurses report medications as "missing" when a medication isn't readily available at the expected time and location.

Description: We conducted a real-time evaluation of missing medication requests to better understand causes and contributing factors. In a previous study, 1 medicine unit (Unit A, 66 beds) and 1 surgical unit (Unit B, 36 beds) were identified as the highest reporters of missing medications at our 455-bed, 24-hour unit dose, cart-fill, urban academic teaching hospital.
Action: This ethics-approved, quality-improvement project assessed "missing dose requests received by the inpatient pharmacy, over 8 prespecified 8-hour periods for the above 2 inpatient units. Study investigators described and tracked the history and pathway of each "missing" medication to determine why it hadn't initially been locatable by the nurse.

Evaluation: A total of 102 missing medication requests were received: 61 and 41 on units A and B, respectively. The distribution of new versus existing medication orders was $66.7 \%$ and $33.3 \%$, respectively. Twentyfour missing medication requests $(23.5 \%)$ were attributable to delivery delays (e.g. medication processed, filled and waiting to be delivered to the floor). Medications for 23 requests $(22.5 \%)$ were easily and immediately found by the study investigators in what would have been the "correct" or "predictable" location according to usual medication delivery processes. Unit transfer issues accounted for 21 requests $(20.5 \%)$. There were 12 requests (11.8\%) for "legitimate" re-issues (e.g. medication fell on floor, bulk items empty). Pharmacy had not dispensed the medication in 12 of the cases $(11.8 \%)$ due to error or system-related oversights. Medications for only 5 requests were untraceable (4.9\%).

Implications: Future initiatives aimed at reducing missing medications will focus on the main contributing factors identified.

\section{Kratom-Induced Acute Liver Failure}

De Francesco $E^{1}$, Lougheed $C^{1}$, Mackenzie $C^{2,3,4}$

${ }^{1}$ London Health Sciences Centre, London, ON

${ }^{2}$ Western University, London, $O N$

${ }^{3}$ Ontario/Manitoba/Nunavut Poison Centre

${ }^{4}$ Hospital for Sick Children, Toronto, ON

Background: The majority of Canadians have used natural health products (NHP) with 12\% reporting an adverse reaction. Substances not approved by Health Canada may be associated with health risks. A case of acute liver injury following exposure to kratom, an unlicensed NHP, is described.

Case Description: A previously healthy young adult presented to the Emergency Department with vomiting, epigastric pain, and transaminitis following recent consumption of kratom, alcohol, and acetaminophen. The ALT, AST, and total bilirubin continued to rise after admission reaching more than 2,700 U/L, 2,900 U/L, and $190 \mathrm{~mol} / \mathrm{L}$, respectively. Blood cultures grew Salmonella javiana. The patient was transferred to the Intensive Care Unit with fulminant liver failure and successfully underwent transplantation.

Assessment of Causality: The patient had a two-day history of heavy alcohol consumption prior to admission. A daily dose of 4 grams of acetaminophen was taken for 3 days following the alcohol binge. Prior to that, 3-4 tablespoons of kratom powder were consumed to boost energy. Urine toxicology and viral hepatitis screens were negative. Wilson disease was ruled out. Given the limited exposures, acetaminophen and alcohol were deemed unlikely to be the cause of the liver failure. Kratom was considered to be a probable provoking agent, as determined by a Naranjo score of 6 .

Literature Review: There have been case reports of cholestatic hepatitis attributed to kratom use in the United States. However, there have been no reports in Canada. Previous cases have reported reversible liver injury following discontinuation of the product. To our knowledge, this is the first report of liver failure necessitating transplantation following recreational use of kratom.

Importance to Practitioners: Health risks associated with unapproved NHPs are not clearly defined. The emergence of toxicity reports with kratom is concerning for patient safety. Practitioners should advise against the use of potentially dangerous products. 


\section{Autoimmune Hepatitis after Receiving One Cycle of Nivolumab/Ipilimumab}

Carter JA, Sax L, Mc Caskill J

London Health Sciences Center, London, ON

Background: Checkpoint inhibitors have been found to cause several autoimmune reactions with varying frequency. Autoimmune hepatitis is a less frequently occurring immune mediated event and often initially presents with nonspecific signs and symptoms. Additionally, the time to onset of immune-mediated adverse effects varies greatly; from days to months.

Case Description: A 59 year old male was diagnosed with metastatic melanoma and was started on palliative ipilimumab and nivolumab. Fifteen days after receiving the first cycle the patient reported numerous symptoms including fatigue, decreased appetite, shortness of breath and intermittent fevers. He was admitted with a presumed diagnosis of sepsis and mildly elevated liver function tests (LFTs), which had been normal at baseline. All cultures returned negative and a rapid rise in LFTs occurred. The symptoms and LFTs resolved after discontinuation of nivolumab/ipilimumab and initiation of dexamethasone.

Assessment of Causality: A Naranjo scale score of 7 indicates that the immunotherapy is the probable cause of hepatitis. The time to onset of symptoms and the LFT increase is consistent with these medications. Additionally, the pattern of LFT elevation is consistent with autoimmune hepatitis and there were no acute ingestions of other substances as alternative causes. Resolution occurred with discontinuation and dexamethasone treatment, which is also consistent with checkpoint inhibitor-induced hepatitis.

Literature Review: Multiple cases of hepatitis with ipilimumab have been reported. However, only 3 publications for each nivolumab and nivolumab/ipilimumab were found upon literature review. To our knowledge, this is the first report for either agent where the reaction occurred after the first cycle.

Importance to Practitioners: Due to increased use, there is a growing importance for practitioners to be aware of checkpoint inhibitor-induced autoimmune reactions and their often nonspecific presentations. In particular, knowledge of the significant variation in onset is important in prompt diagnosis and prevention of lasting complications.

\section{Characterization of Medication-Related Near Miss Safety Events}

Moreno M, Zuberi $M$

Toronto General Hospital, University Health Network, Toronto, ON

Background: Hospitals require medication-related Safety Events to be reported to prevent harm to both patients and staff. The 2004 Canadian Adverse Events study by Baker et al identified that $1 / 3$ of hospital adverse events were preventable. Adverse events related to medications and fluid were the second most common type. Safety Events can be classified as Near Miss Safety Events, Precursor Safety Events, and Serious Safety Events. Near Misses have not reached the patient and have not caused harm. They are often identified through a detection barrier in place to prevent the error. By analyzing these events we can learn how to prevent more serious events in the future.

Description: To characterize Medication-Related Near Miss Safety Events (MRNMSE) reported at a 433 bed teaching hospital between 1 Jan 2017 to 31 Dec 2017.

Action: A retrospective review of MRNMSE was conducted for the specified time period. Specifically, MRNMSE were characterized as pharmacy-related and if pharmacy-related then subcategorized as repackaging errors, dispensing/supply errors, workflow errors, or other pharmacy-related errors.

Evaluation: In total, 175 MRNMSE were reviewed. Of these $41.1 \%$ $(72 / 175)$ were pharmacy-related and are characterized in the Table. Of pharmacy-related MRNMSE 22.9\% (40/175) were dispensing/supply errors. Common contributors identified were wrong drug/same strength, right drug/wrong strength, and right drug/wrong route.

Implications: Although 72 MRNMSE were characterized as pharmacyrelated, the overall incidence was low since more than 3 million oral doses were dispensed during the same time period. Repackaging Errors and Dispensing/Supply Errors were both reported by nurses when using automated dispensing cabinets so developing detection barriers to prevent Safety Events related to loading/refilling automated dispensing cabinets could be beneficial.

For the table that goes with this abstract, please see Abstract Appendix, available at https://www.cjhp-online.ca/index.php/cjhp/issue/view/188/ show Toc

\section{Incidents Associated with Centralized Automated Processing of Multi-Medication Compliance Packs}

Boucher $A^{1,2}$, Sheng $L^{3}$, Ho $C^{1,2,3}$, Tolios $P^{4}$, Almaula $K^{4}$, Wong $J^{4}$

${ }^{1}$ Institute for Safe Medication Practices Canada, Toronto, ON

${ }^{2}$ Leslie Dan Faculty of Pharmacy, University of Toronto, Toronto, ON

${ }^{3}$ School of Pharmacy, University of Waterloo, Waterloo, ON

${ }^{4}$ CPAK Shared Services, Shoppers Drug Mart, Toronto, ON

Background: Studies have found that manual preparation of multimedication compliance packs is associated with significant risk of medication incidents. Centralized automated prescription processing is a future trend in pharmacy.

Description: The purpose of this study was to characterize and analyze medication incidents associated with automated preparation of compliance packs at the largest centralized prescription filling facility in Canada.

Action: We performed descriptive statistics and qualitative thematic analysis on incidents associated with 10 automated compliance pack preparation machines (i.e. robots) reported by pharmacy professionals at the centralized filling pharmacy from December 2017 to January 2018.

Evaluation: A total of 121,250 compliance packs were prepared during the study period, of which $4.82 \%$ was associated with an incident. The most common types of incidents were "pill jump" (18.89\%), "additional pill" (18.29\%), and "missing pill" (15.52\%). Incidents were categorized into three main themes: manual processes; equipment maintenance; and implementation of standard operating procedures. Recommendations included automation of human-involved processes, review of current policies and procedures, education/training of staff, and fine-tuning of machine/robot performance.

Implications: We found a 33\% reduction in incident rates with centralized automated processing of compliance packs when compared to what was reported for manual preparation in the literature. Areas of improvement in a centralized automated prescription filling pharmacy should focus on reducing human errors, improving robot function, and enforcing staff compliance with standard operating procedures. 


\section{Chemical Stability of Cloxacillin in Sterile Water for Injection (SWFI) Stored in Polypropylene (PP) Syringes (50 and $100 \mathrm{mg} / \mathrm{mL}$ ) and Glass Vials $(250 \mathrm{mg} / \mathrm{mL})$ at $4^{\circ} \mathrm{C}$ and $25^{\circ} \mathrm{C}$}

Perks $W^{1}$, Marchesano $R^{1}$, Law $S^{1}$, Iazzetta J', Walker $S E^{2}$

${ }^{1}$ Department of Pharmacy, Sunnybrook Health Sciences Centre, Toronto, ON

${ }^{2}$ Leslie Dan Faculty of Pharmacy, University of Toronto, Toronto, ON

Background: Interruptions in minibag supplies necessitated conservation strategies, including compounding cloxacillin in syringes and administration by direct IV.

Objective: To evaluate the chemical stability of cloxacillin reconstituted with Sterile Water for Injection (SWFI) at concentrations of $250 \mathrm{mg} / \mathrm{mL}$ in glass vials and 50 and $100 \mathrm{mg} / \mathrm{mL}$ in PP syringes at $4^{\circ} \mathrm{C}$ and $25^{\circ} \mathrm{C}$.

Methods: On study day $0,10 \mathrm{~g}$ vials of cloxacillin were reconstituted with $40 \mathrm{~mL}$ of SWFI $(250 \mathrm{mg} / \mathrm{mL})$. Vials were further diluted with SWFI to achieve concentrations of 50 and $100 \mathrm{mg} / \mathrm{mL}$ and drawn into PP syringes. Vials and syringes were stored at $4^{\circ} \mathrm{C}$ and $25^{\circ} \mathrm{C}$. Cloxacillin concentration analysis was completed on study days $0,1,2,4,7,10,14,17$ and 21 using a validated stability-indicating liquid chromatographic method with UV detection. Chemical stability was based on the intersection of the lower limit of the $95 \%$ confidence interval of the observed degradation rate and the time to achieve $90 \%$ of the initial concentration (T-90).

Results: The analytical method separated degradation products from cloxacillin and measured the concentration specifically, accurately and reproducibly $(0.56 \%[\mathrm{CV}(\%])$. During the study period all solutions retained more than $90 \%$ of the initial concentration for the entire study period (14 days) at $4^{\circ} \mathrm{C}$ and for 24 hours at $25^{\circ} \mathrm{C}$. Multiple linear regression revealed significant differences in percent remaining due to study day $(p<0.001)$ and temperature $(p<0.001)$ but not concentration $(\mathrm{p}=0.679)$ or container $(\mathrm{p}=0.803)$. The calculated T-90, with $95 \%$ confidence exceeded 14 days for all concentrations at $4^{\circ} \mathrm{C}$ and 1 day at $25^{\circ} \mathrm{C}$.

Conclusions: This study demonstrated that cloxacillin solutions in vials $(250 \mathrm{mg} / \mathrm{mL})$ and syringes $(50$ and $100 \mathrm{mg} / \mathrm{mL})$ can be stored for up to 14 days if continuously stored at $4^{\circ} \mathrm{C}$ and 1 day at $25^{\circ} \mathrm{C}$. If the syringes are exposed to room temperature, the maximum storage is 7 -days at $4^{\circ} \mathrm{C}$, allowing 12 hours exposure at $25^{\circ} \mathrm{C}$ during this 7 -day period.

\section{Stability of $1.0,0.2$ and $0.025 \mathrm{mg} / \mathrm{mL}$ Milrinone Solutions Stored in Syringes at $4^{\circ} \mathrm{C}$ and at Room Temperature $\left(25^{\circ} \mathrm{C}\right)$}

Hook $R^{1}$, Riss $V^{1}$, Scharrer $E^{1}$, Law $S^{2}$, Walker $S E^{3}$

${ }^{1}$ Departments of Pharmacy, Hospital for Sick Children, Toronto, ON

${ }^{2}$ Sunnybrook Health Sciences Centre, Toronto, ON

${ }^{3}$ Leslie Dan Faculty of Pharmacy, University of Toronto, Toronto, ON

Background: Inpatient hospital pharmacies must compound intravenous products and assign an appropriate beyond-use-date (BUD) as per NAPRA standards. While previous publications have demonstrated the stability of milrinone, data for lower pediatric concentrations stored in syringes for more than 14 days is not available.

Objective: To evaluate the chemical stability milrinone prepared in syringes at concentrations of $1 \mathrm{mg} / \mathrm{mL}$ (undiluted), 0.2 and $0.025 \mathrm{mg} / \mathrm{mL}$ (diluted in either $0.45 \%$ sodium chloride or $5 \%$ dextrose in water (D5W)) at both room temperature and in the refrigerator.

Methods: On study day 0, 60mL solutions of $1,0.2$ and $0.025 \mathrm{mg} / \mathrm{mL}$ concentrations of milrinone were prepared in $60 \mathrm{~mL}$ BD syringes. 3 units of each container and concentration were stored at room temperature (25C) and 3 were stored at 4C. Concentration analysis was completed on study days $0,1,7,14,21,28,42,54,75$ and 90 using a validated stability-indicating liquid chromatographic method with UV detection. Chemical stability was based on the intersection of the lower limit of the $95 \%$ confidence interval of the observed degradation rate and the time to achieve $90 \%$ of the initial concentration (T-90).

Results: The analytical method separated degradation products from milrinone such that the concentration was measured specifically, accurately (deviations from known averaged 2.24\%) and reproducibly (replicate error averaged $0.66 \%(\mathrm{CV}(\%))$. During the study period all solutions retained more than $98.14 \%$ of the initial concentration. Multiple linear regression revealed significant differences in percent remaining due to study day $(\mathrm{p}=0.008)$ and temperature $(\mathrm{p}=0.0276)$ but not concentration $(\mathrm{p}=0.108)$ or diluent $(\mathrm{p}=0.635)$. The calculated T-90, with 95\% confidence, exceeded 155.61 days for all concentrations, temperatures and diluents.

Conclusions: We conclude of $1 \mathrm{mg} / \mathrm{mL}$ (undiluted), 0.2 and $0.025 \mathrm{mg} / \mathrm{mL}$ diluted in either $0.45 \%$ sodium chloride or D $5 \mathrm{~W}$, stored at either room temperature or in the refrigerator in polypropylene BD syringes are physically and chemically stable for 90 days.

\section{Stability of Magnesium Sulphate Solutions in PVC Minibags, Non-PVC Minibags, and Polypropylene Syringes Stored at $4^{\circ} \mathrm{C}$}

Tran C-P $P^{1,2}$, Facca $N^{1}$, Smith $N^{3}$

${ }^{1}$ Department of Pharmacy, London Health Sciences Centre (LHSC),

London, $O N$

${ }^{2}$ School of Pharmacy, University of Waterloo, Kitchener, ON

${ }^{3}$ Department of Pathology \& Laboratory Medicine, LHSC, London, ON

Background: To our knowledge, no stability data exist for magnesium sulphate solutions in plastic bags and syringes.

Objectives: To test the physical and chemical stability of 10 and $143 \mathrm{mg} / \mathrm{mL}$ magnesium sulphate solutions stored at $4^{\circ} \mathrm{C}$ for 14 days in polyvinyl chloride (PVC) minibags, non-PVC minibags and polypropylene syringes.

Methods: The bags and syringes containing the magnesium sulphate solutions were stored at $4^{\circ} \mathrm{C}$ for 14 days. Samples were taken daily and frozen at $-20^{\circ} \mathrm{C}$, pending analysis. Samples from selected days through the study were analysed in quintuplicate on a Roche Cobas C 701 analyzer. Magnesium concentration means from each selected day were compared to those of Day 0 by calculating them as a percentage of the Day 0 value. Chemical stability was demonstrated if lower limit of $95 \%$ confidence interval did not fall below $90 \%$ of the Day 0 value. Physical stability was assessed by daily colour and clarity checks.

Results: One magnesium sulphate solution, $10 \mathrm{mg} / \mathrm{mL}$ magnesium sulphate in $0.9 \%$ sodium chloride in the PVC minibag, performed similarly to all of the other solutions up to Day 13, but on Day 14 the $\%$ of Day 0 value fell to $75 \%$. The lower limits of the $95 \%$ confidence intervals of all of the other solutions remained above $90 \%$ of the Day 0 concentration for the entire 14 day study. All of the magnesium sulphate solutions remained clear and colourless throughout the study.

Conclusions: All magnesium sulphate solutions were physically stable for 14 days. All magnesium sulphate solutions except the $10 \mathrm{mg} \mathrm{mL}$ solution in $0.9 \%$ sodium chloride in the PVC minibag, were chemically stable stored at $4^{\circ} \mathrm{C}$ for 14 days, but all solutions were chemically stable for 9 days. 


\section{Stability of Cardioplegia Additive Solution in Polyvinyl Chloride Bags Stored at $4^{\circ} \mathrm{C}$ for 9 Days}

Tran C-P ${ }^{1,2}$, Katyukha $A^{3}$, Facca $N^{1}$, Smith $N^{4}$

${ }^{1}$ Department of Pharmacy, London Health Sciences Centre (LHSC),

London, $O N$

${ }^{2}$ School of Pharmacy, University of Waterloo, Kitchener, $O N$

${ }^{3}$ Department of Physiology and Pharmacology, Western University (UWO), London, $O N$

${ }^{4}$ Department of Pathology \& Laboratory Medicine, LHSC/UWO,

London, $O N$

Background: To our knowledge, no stability data exist for the cardioplegia additive solution (adenosine $0.12 \mathrm{mg} / \mathrm{mL}$, dextrose [glucose] $150 \mathrm{mg} / \mathrm{mL}$, lidocaine $2 \mathrm{mg} / \mathrm{mL}$, magnesium sulphate $200 \mathrm{mg} / \mathrm{mL}$ ).

Objectives: To test the physical and chemical stability of the cardioplegia additive components, (except insulin) when stored at $4{ }^{\circ} \mathrm{C}$ in polyvinyl chloride (PVC) minibags for 9 days.

Methods: The cardioplegia additive solution was stored in minibags at $4^{\circ} \mathrm{C}$ for 9 days, with samples taken daily and frozen at $-84^{\circ} \mathrm{C}$, pending analysis. Samples from selected days through the study were analysed in quintuplicate. Glucose and magnesium concentrations were measured on a Roche Cobas C 701 analyzer. Adenosine and lidocaine were measured by gas chromatography/mass spectrometry, with each drug having its own internal standard (ISTD). From each measurement, drug/ISTD peak area ratios (PARs) were calculated for adenosine and lidocaine. Component concentrations from each selected day were compared to those of Day 0 by calculating them as a percentage of the Day 0 value. Chemical stability was demonstrated if lower limit of $95 \%$ confidence interval did not fall below $90 \%$ of the Day 0 value. Mass spectra of Day 9 drug peaks were compared to those of Day 0 peaks, to confirm purity of the measured drug peaks. Physical stability was assessed by daily colour and clarity checks.

Results: None of the lower limits of $95 \%$ confidence intervals of any components fell below $90 \%$ of the Day 0 value. Day 9 mass spectra of lidocaine and adenosine were identical to those of Day 0 . The cardioplegia solution remained clear and colourless throughout the study.

Conclusions: All measured cardioplegia components were physically and chemically stable for 9 days. This compound will be assigned a beyond-use date of 9 days.

\section{Review of Microbial Contamination of Vials Used for Compounding with Closed System Drug Transfer Devices}

Soubieux $A^{1}$, Tanguay $C^{1}$, Bussières J-F $F^{1,2}$

${ }^{1}$ Unité de Recherche en Pratique Pharmaceutique, Sainte-Justine

University Hospital, Montréal, QC

${ }^{2}$ Faculty of Pharmacy, Université de Montréal, Montréal, QC

Background: Vials that are punctured under ISO5 conditions must have a beyond-use date of 6 hours, per the United States Pharmacopeia chapter $<797>$ to ensure their sterility. Some institutions are using closed system drug transfer devices (CSTD) when compounding sterile antineoplastic drugs. CSTDs offer a closed environment, so authors are arguing that the sterility is maintained and thus extend the beyond-use date.

Objective(s): To perform a literature review on microbial contamination of vials used for compounding antineoplastic drugs with CSTDs.

Methods: A literature review was performed on 2018/08/08. The following terms were searched on PubMed, Embase, CINALH: CSTD and beyond-use date. The proceedings of three relevant conferences were searched (Groupe d'évaluation et de recherche sur la protection en atmosphère contrôlée from 2013-2017, Professional Practice Conference 2013-2018 and Canadian Association of Pharmacy in Oncology 2016-2018). We included any study that presented results on microbial contamination following sterile compounding with CSTDs.

Results: A total of 397 studies were found and 13 met our inclusion criteria. A total of 1392 vials were tested, 1320 vials using a CSTD ( $n=11$ studies) and 72 vials without using a CSTD ( $\mathrm{n}=3$ studies). The microbial contamination was mainly evaluated at varying time after initial puncture of the vial, 24 hours ( $n=8$ studies), 48 hours ( $n=6$ studies), 72 hours ( $n=6$ studies), 7 days ( $n=7$ studies), 14 days ( $n=3$ studies). Five studies showed no microbial contamination. No study showed a significant difference in the percentage of contamination with and without using a CSTD.

Conclusion(s): The majority of antineoplastic drugs vials used for compounding under sterile conditions showed little or no microbial contamination. Future studies should compare microbial contamination with and without a CSTD, to validate the potential added benefit of CSTDs on maintaining sterility, when compounding is performed with an aseptic method.

\section{Estimating the Proportion of Emergency Depart- ment Visits That Can Be Managed by Pharmacists' Expanding Scope in Ontario between 2010 and 2017}

Alsabbagh MW, Houle SKD

School of Pharmacy, University of Waterloo, Kitchener, ON

Background: Pharmacists' scope of practice has expanded significantly in Canada, which may help alleviate emergency department (ED) workload for minor conditions and prescription renewals. Previous research revealed that a considerable percentage of ED visits are not urgent and can be managed by primary health care activities. However, it is not known how many of these cases can be managed by pharmacists within expanded scope of practice.

Objectives: The aims of this study are to: (1) Determine the proportion of ED visits that can potentially be managed by pharmacists within their expanded scope of practice, and (2) Determine the most prevalent conditions within these cases that can be managed by pharmacists.

Methods: This is a retrospective quantitative longitudinal cohort study using administrative record-level databases provided by the Canadian Institute of Health Information (CIHI) from 2010 to 2017. Among all unscheduled ED visits in Ontario, we identified all visits with a Family Practice Sensitive Condition (FPSC) and Canadian Triage and Acuity Scale (CTAS) score of 4 ("Less Urgent") or 5 ("Non-Urgent"). We then identified a list of conditions that can be managed by pharmacists using the broadest scope available in Canada.

Results: Among the 34,550,020 ED visits identified, $12.4 \%$ $(\mathrm{n}=4,293,807)$ were considered FPSC with CTAS 4 or 5 . Of these, $1,494,887(34.8 \%)$ were for conditions considered to be manageable by pharmacists, representing $4.3 \%$ of all ED visits. The most frequent diagnoses that can be managed by pharmacists were: acute pharyngitis, conjunctivitis, rash and other nonspecific skin eruption, otitis externa, cough, acute sinusitis, and dermatitis.

Conclusions: Under an expanded scope, pharmacists can manage a significant proportion of unnecessary ED visits. The introduction of ED-based pharmacists practicing under an expanded scope, or greater expansion of scope for community pharmacists, may have a positive impact on overcrowding in Canadian emergency departments. 


\section{The Impact of Pharmacist Medication Management on 30-Day Hospital Re-Admission Rates as a Member of a Rapid Response Transitional Team}

\author{
Chu C, Vanstone G, Thompson J, Newman P, Dhaliwall S \\ NorthWest Telepharmacy Solutions, Winnipeg, MB
}

Background: Unplanned hospital admissions are associated with increased patient mortality and health care costs. In Canada, 1 in 11 patients are readmitted within a month of leaving hospital. Several factors are associated with hospital re-admissions and deemed preventable. Including pharmacists to conduct medication reviews at discharge and follow-up with the primary care physician and pharmacy can lead to reduced hospital admissions. Nurse-led Rapid Response Transitional Teams (RRTT) have been created to ensure patients thrive out of hospital, prevent hospital re-admissions, however very few of these teams include a pharmacist.

Description: A RRTT incorporated a team of 8 pharmacists to conduct in-person medication management (MM) for medically complex patients in their homes. Patients referred to the RRTT were recently discharged from hospital or referred by their family practitioner and deemed medically complex.

Action: The RRTT nurse identified patients to be referred to a pharmacist for in-home MM. The pharmacist provided MM: medication reconciliation, identification of medication discrepancies and drug-related problems, patient counselling/coaching/education and medication disposal, provision of health literacy and follow up with the patient's primary care physician and pharmacy. The pharmacist utilized the electronic health record (EMR), and community pharmacy medication histories and other resources/tools as required. The encounter and recommendations were documented in the RRTT EMR.

Evaluation: Patients who were seen by the pharmacist had a lower 30 -day readmission rate (17\%) compared to patients who did not (21\%). The majority of patients (94\%) were seen within 30 days of the RRTT referral.

Implications: Integrating pharmacists on a RRTT to conduct in-home MM can lead to a decrease in 30-day hospital readmissions.

For the table that goes with this abstract, please see Abstract Appendix, available at https://www.cjhp-online.ca/index.php/cjhp/issue/view/188/ showToc

\section{Do Knowledge Gaps about Opioids Exist for Pharmacists? A Closer Look at a Novel Online Learning Platform}

\section{Bancsi A, Grindrod K, Killeen R}

\section{University of Waterloo School of Pharmacy, Kitchener, ON}

Background: An online learning platform was developed to help pharmacists and pharmacy technicians build skills in various areas. In May 2018 in the context of an ongoing national opioid crisis, we developed a module to help pharmacists improve their ability to assess and manage opioid prescriptions. Funding was provided by a provincial college of pharmacists.

Objective: To evaluate pharmacists' level of knowledge and skill in assessing and managing opioid prescriptions, using data collected from a novel online learning platform.

Methods: We developed an online, quiz-based, multimedia learning platform. Pharmacy practice and therapeutics topics are presented as separate modules. The opioid module, based on national guidelines, includes five learning objectives, seven quizzes, two animated videos, two infographics, and reminder flashcards. It also links to evidence-based resources from the Canadian Pharmacists Association and the Centre for Effective Practice. De-identified quiz responses were downloaded from the platform, following an ethics approval. Each quiz question was coded to its respective learning objective to generate a mean score. Descriptive statistics were used to summarize the data.

Results: A total of 645 users completed one quiz in the module, and 200 completed the entire module. Most users were female (68\%), licensed pharmacists $(71 \%)$, trained in Canada (67\%), practising in Ontario $(90 \%)$ and had been practising an average of 13 years. Out of 5 learning objectives, users performed best on understanding how and when to start, switch, taper, and stop opioid therapy (mean score 64\%). Users performed worst on identifying signs and symptoms of opioid adverse effects (mean score 55\%) and making recommendations for management (mean score 55\%).

Conclusion: Future education initiatives should focus on helping pharmacists improve in all areas of opioid prescribing and management, with a heavier focus needed on assessment and management of adverse effects and making recommendations for changes in therapy.

\section{Pharmacist Medication Reviews via Videoconference: A Prospective Cohort Study Pilot Study in Remote and Rural Underserviced Communities}

Newman P, McDonald K, Dhaliwall S, Chan D NorthWest Telepharmacy Solutions, Winnipeg, MB

Background: Canada has a publicly funded universal healthcare system, but not all residents have the same access to care, including that of a pharmacist. While urban areas have nearby pharmacies, many small communities have medications shipped to a local nursing station/ hospital. Pharmacist medication reviews (PMR) in the primary care setting improve health outcomes. For patients in isolated regions, without a local pharmacist, PMR is non-existent. Multidisciplinary telemedicine teams including pharmacists have demonstrated improved health-related outcomes. Despite rapid growth of videoconferencing (VC) in remote communities for improved access to healthcare, telepharmacists continue to be underutilized.

Description: This prospective observational cohort pilot study included interviewing patients from two remote communities eligible for a PMR (at least 3 medications for chronic conditions or diagnosed with diabetes) at their hospital/nursing station via VC.

Action: The telepharmacist reviewed patient electronic sources of medication lists/medical history and identified drug-therapy problems (DTP). A motivational interviewing approach was taken -collaborative, evocative, and the honoring of patient autonomy by listening, asking, and informing. Identified DTP's were discussed with the patient and/ or their prescribers. Following the interview, patients completed a PMR satisfaction questionnaire. DTP's were documented using the PCNE V6 classification system.

Evaluation: One half of all patients were eligible for PMR. Of those that were contacted, $85 \%$ agreed to participate and $40 \%$ attended the PMR. Mean times required to prepare, interview, and follow up were 13, 21 , and 12 minutes respectively with 1.3 DTP identified and 10.5 medications per patient. Patients reported a $71 \%$ positive response rate in support of PMR by VC. Inefficiencies, barriers and facilitators were described.

Implications: Utilizing established VC technology to conduct PMR is acceptable by patients and offers an opportunity to address a significant disparity in the provision of health care in remote communities without in-person access to a pharmacist. 


\section{Implementation of Electronic Special Access Program Forms to Improve Workflow for Pharmacists}

Dewhurst $N F^{1,2}$, Lieu $J^{1}$, Tom $E^{1}$, Chen WXY' $Y^{2}$ Chan $S C^{2}$, Chang $C^{2}$, Chant $C^{1,2}$

'St. Michael's Hospital, Pharmacy Department, Toronto, ON

${ }^{2}$ Leslie Dan Faculty of Pharmacy, University of Toronto, Toronto, ON

Background: Health Canada's Special Access Program (SAP) allows practitioners to request access to drugs that are not available for use in Canada. While SAP request forms must be completed by the practitioner, pharmacists often facilitate and assist with SAP form completion, requiring significant time, impacting pharmacist workload. Pharmacists also report that paper forms are obsolete and electronic forms would expedite the process for both practitioner and pharmacist.

Description: As part of meeting the standards for hospital accreditation and improving workflow for pharmacists, a standardized process was needed for SAP drugs. A policy was implemented in June 2016 for patient-specific SAP requests and prescription process for inpatients.

Action: Common SAP drugs were identified and pre-built electronic forms were created. SAP workflow was developed for practitioners and pharmacists to ensure efficiency in the completion of SAP drug requests. This workflow was also integrated with the computerized physician order entry (CPOE) system to guide practitioners when ordering of SAP drugs is required. A survey, including a Likert Scale, was used to evaluate the usage of pre-built electronic SAP forms and the pharmacists' perception of the policy on their practice.

Evaluation: Fifteen pharmacists that commonly complete SAP request forms responded to the survey. The average percentage of prescribers that independently completed SAP forms increased from $25 \%$ to $58 \%$. The average total time pharmacists spent on completing SAP requests within a month decreased from $115 \mathrm{~min}$ to $87 \mathrm{~min}$. Survey responses indicated that a majority of pharmacists thought that the SAP completion process became overall easier to complete.

Implications: The results suggest that pharmacists working within inpatient units have benefitted from pre-built electronic SAP forms, reducing time spent on SAP requests and making the overall process easier. In the future, forms for other SAP drugs should be implemented.

\section{Use of Extensive Auditing to Reduce Potential Diversion of Narcotics and Controlled Drugs in a Healthcare Facility}

\section{Emerey J, Shabeen $M$, Adams $C$ \\ Cornwall Community Hospital, Cornwall $O N$}

Background: Fueled by the opioid epidemic, narcotic and controlled drug (NCD) diversion is unfortunately a common occurrence in Canadian healthcare facilities. Media investigations have outlined the extent of this diversion. More and more, healthcare facilities are being held accountable to ensure the medication distribution process for NCDs is rigorous in preventing diversion.

Description: Following the implementation of a new electronic medical record (EMR) and computerized physician order entry (CPOE) system with electronic medication administration records (eMAR), our facility began conducting numerous audits around the distribution of NCDs.

Action: Several processes were implemented to monitor for diversion: daily reconciliation for any NCDs dispensed by override; monthly graph reports for specific injectable NCDs; random oral NCD usage reports; and checks against charts of discharged patients, which are still accessible for 24 hours after discharge. In addition to random user audits, should usage for any user be outside of range, user-specific audits are performed. Routine user audits reconcile dispenses taken against the medication order. The selection for these is chosen at random and includes both high and low volume users. Pharmacy transactions are also subjected to auditing. NCDs stocked in the automated dispensing units (ADU) are compared with the quantities removed from the department. NCDs retrieved from ADU return drawers are reconciled with quantities re-stocked or returned to pharmacy.

Evaluation: Over a one year span, medication incidents involving NCD discrepancies decreased by 98\%, from 50 in July 2017 to 1 in June 2018. Audits and monitoring are ongoing.

Implications: Establishing an intensive auditing process improves the detection of and reduces the opportunity for diversion in a healthcare facility. The adoption of a new EMR with eMAR allowed our site to easily obtain the information required to implement this process. We believe that these results could be replicated at other healthcare facilities.

\section{An Analysis of Medication Returns to Inpatient Pharmacy Using a Closed-Loop Health Information System}

Hasan S, He T, Burger C, Kalidoss S, Lukinuk C St. Joseph's Healthcare Hamilton, Hamilton, $O N$

Background: Processing medication returns is a challenging task for many acute care pharmacies. Medication returns greatly increase the workload for pharmacists and technicians, and significantly impact inventory. However, few studies have analyzed the impact of medication returns on hospital pharmacies.

Description: In December 2017, a tertiary care hospital implemented a new health information system (HIS), with closed-loop medication administration and electronic inventory tracking, incorporating perpetual inventory. Pharmacy staff were required to electronically process returns into the HIS, prior to returning unit-dosed medications to stock. This project was conducted to analyze the number of medications returned, assess the workload impact of the new process, and determine the common reasons for returns.

Action: The project consisted of two parts. Firstly, a retrospective analysis of medication returns was conducted using HIS reports to determine the number of returns, identify the commonly-returned medications, and measure the time spent by pharmacy staff processing returns. Secondly, a cross-sectional analysis was conducted to characterize the reasons medications were returned from select high-volume inpatient units. Data was analyzed using Microsoft Excel.

Evaluation: Out of 117,834 dispensed unit-doses, 30,299 units (26\%) were returned to the pharmacy over a 22 -day period. On average per day, 1,364 unit-doses were returned, and an estimated 4 hours was spent processing returns. Commonly-returned medications included: senna (5.8\%), dalteparin (3\%), polyethylene glycol $(2.3 \%)$, cholecalciferol $(1.9 \%)$, pantoprazole $(1.9 \%)$, vancomycin (1.8\%), furosemide injectable $(1.8 \%)$ and tablets (1.7\%). Reasons for returns included: discontinuation of therapy (19\%), patient/family refused (13\%), patient discharged from unit $(7 \%)$, nurse professional opinion $(7 \%)$, or unable to determine (8\%).

Implications: Medication returns add a significant workload to hospital pharmacies. Closed-loop HIS provide tools for pharmacy administrators to understand the reasons for returned medications, identify workflow efficiencies, and improve the medication use process. 


\section{Utilization of the Electronic Health Record to Minimize Pharmacy Alert Fatigue}

Hollis $K^{1}$, Wong $C^{2}$, Wong $E^{1}$, Corrigan $S^{1}$, Trinneer $A^{1}$, Girgis $P^{2}$, Fung $\mathcal{F}^{2}$, Vaillancourt $R^{1}$

${ }^{1}$ Children's Hospital of Eastern Ontario, Ottawa, ON

${ }^{2}$ Hospital for Sick Children, Toronto, ON

Background: In 2017 and 2018, two institutions collaborated on the implementation of one single electronic health record. Pharmacists were reporting high numbers of clinically irrelevant alerts firing during order validation.

Description: The electronic health record allows for analysis of the types of alerts fired and Pharmacist response during order validation.

Action: The alerts were reviewed looking at the frequency of firing and clinical relevance. The Medication Safety Committee reviewed this analysis and made decisions to filter out the clinically irrelevant alerts identified. This practice was completed in collaboration with both institutions.

Evaluation: Drug-drug interactions accounted for $44.1 \%$ of all medication warnings. Duplicate therapy class alerts represented $17.7 \%$ of all medication warnings fired.

Implications:

The following alerts were suppressed:

Opioids (Immediate Release) / Benzodiazepines

Aminoglycosides / Penicillins

NSAIDs/Corticosteroids

Antihistamine

Parenteral solutions containing sodium

For the tables that go with this abstract, please see Abstract Appendix, available at https://www.cjhp-online.ca/index.php/cjhp/issue/view/188/ show Toc

\section{An Assessment of the Cost-Effectiveness of 24/7 Hospital Pharmacy}

Peng $M^{1,2}$, Pradhan $R^{2}$, Ritchie $M^{2}$, Sullivan $S^{2}$, Carating $H^{2}$, Walker $S^{1,2}$ ${ }^{1}$ Leslie Dan Faculty of Pharmacy, University of Toronto, Toronto, ON

${ }^{2}$ Department of Pharmacy, Sunnybrook Health Sciences Centre, Toronto, ON

Background: Regulatory bodies such as the Ontario College of Pharmacists and Accreditation Canada have published standards that require the pharmacist to review all prescriptions prior to administration of the first dose. While this is the norm in the community, it is not always the case in hospital practice.

Objective: This study is aimed to determine the cost-effectiveness of 24/7 hospital pharmacy.

Methods: All orders were collected in a defined time-period and the time when orders were written was recorded to produce a 24-hour order distribution curve. Sub-analysis included classification of orders based on weekdays, weekend, urgency, and location. The average number of orders per hour a pharmacist can process was determined to establish a benchmark workload indicator. Based on an average pharmacists' salary including benefits, the cost per order verification was determined. This cost and workload analysis informed the 24-hour pharmacy decision.

Results: A total of 17,385 orders were included in the analysis, of which 2921 were overnight orders. Overnight workload mostly came from wards $(37.9 \%)$, emergency $(34.4 \%)$ and ICU areas (11.1\%). For weekdays, $70 \%$ of the urgent overnight orders were written between 8PM-12AM, while 50\% were written between 4PM-8PM on a weekend. The cost per order verification for weekdays and weekend averaged $\$ 0.73$ and $\$ 0.76$, respectively, indicating that the average order-entry pharmacist workload is 70 and 67 orders per hour on weekdays and weekend, respectively. Between 12AM-7AM the number of orders would only justify 0.36 FTE, while between 8PM-12AM, workload supports 0.72 FTE. Similar observations were made on the weekend. The number of orders between 8PM-7:30AM does not exceed the workload of 1 FTE pharmacist at each hour for weekdays or weekends.

Conclusion: The data failed to support 24/7 pharmacy operation as there was insufficient workload to justify a pharmacist's wage. However, the data did support extending pharmacy hours.

\section{Identification and Selection of Preferred Candidates for the Position of Chief Executive Officer of a Professional Association}

Adams $C^{1}$, Doucette $D^{2}$, Dumont $Z^{3}$, Fitch $P^{4}$, Jennings $D^{5}$, Lyder $C^{6}$, Millin $B^{7}$

${ }^{1}$ Cornwall Community Hospital, Cornwall, ON

${ }^{2}$ Horizon Health Network, Moncton, NB

${ }^{3}$ Saskatchewan Health Authority, Regina, SK

${ }^{4}$ Winnipeg Regional Health Authority, Winnipeg, $M B$

${ }^{5}$ Sault Ste Marie, $O N$

${ }^{6}$ CSHP, Edmonton, $A B$

${ }^{7}$ Provincial Health Services Authority, Vancouver, BC

Background: A chief administrator of a professional association is responsible for the leadership and management of the association in accordance with the strategic direction. Succession of such a position is of critical importance.

Description: The current chief administrator of the Canadian Society of Hospital Pharmacists (CSHP) was approaching retirement, and a successor was needed. The CSHP board of directors commissioned a Search and Selection Task Force (SSTF) to undertake the identification and selection of preferred candidates.

Action: After review and adaptation of association management best practices for recruitment of a chief administrator, the SSTF: reviewed and revised the job description; developed and advertised the job posting; performed initial screen of applications and $\mathrm{CV}$ s to ensure job requirements met; developed and implemented an independent ranking tool for those meeting qualifications; developed an interview guide and performed initial interviews of top candidates; developed and implemented an independent ranking tool for the first round of interviews; developed and implemented reference check guide with identified referees; ranked top candidates; developed an interview guide and coordinated top ranking candidates for a second interview conducted by the board.

Evaluation: Following the job description review, the position title was changed from executive director to chief executive officer (CEO). The job posting was advertised on three websites, with the intent of reaching an appropriate target audience. Thirty-two applications were received. Following initial review, 20 met qualifications and were ranked. The top five candidates were selected for the first round of interviews. Twelve reference checks were performed. Two candidates were selected for the second round interview with the board.

Implications: The recruitment and selection process led to an abundance of qualified candidates applying, a surplus of successful candidates at each step of the screening process, and the SSTF was successful in its mission to identify and select preferred candidates for the position of CEO. 


\section{Comparison of Preventive Cardiovascular Pharmacotherapy in Surgical versus Percutaneous Coronary Revascularization}

Barry $A R^{1,2}$, Wang $E^{3}$, Chua $D^{3}$, Pearson GJ

${ }^{1}$ Faculty of Pharmaceutical Sciences, University of British Columbia, Vancouver, $B C$

${ }^{2}$ Chilliwack General Hospital, Lower Mainland Pharmacy Services, Chilliwack, $B C$

${ }^{3}$ St. Paul's Hospital, Lower Mainland Pharmacy Services, Vancouver, BC ${ }^{4}$ Faculty of Medicine o Dentistry (Division of Cardiology), University of Alberta, Edmonton, $A B$

Background: Data suggest patients who undergo coronary artery bypass graft surgery $(\mathrm{CABG})$ have a lower rate of preventive cardiovascular pharmacotherapy use compared to percutaneous coronary intervention (PCI). However, these studies do not account for justified non-use (e.g., allergy/intolerance/contraindication).

Objective: To assess rate of utilization of preventive cardiovascular pharmacotherapy at discharge in CABG versus PCI patients post-acute coronary syndrome (ACS).

Methods: Prospective cohort study was conducted at St. Paul's Hospital in Vancouver, British Columbia. Consecutive patients aged $\geq 18$ years discharged post-ACS after CABG or PCI between January-June 2018 were included. Data collected included demographics, revascularization strategy, and preventive cardiovascular medication use specifically acetylsalicylic acid (ASA), P2Y12 inhibitors, beta-blockers, angiotensinconverting enzyme inhibitors/angiotensin receptor blockers (ACEI/ ARBs) and statins including adjustment for justified non-use. Statistical analyses included t-test and chi-square test for continuous and categorical variables, respectively, with a significance level of $<0.05$.

Results: One hundred and sixty patients were included. Mean age was 65 years and $83 \%$ were male. Comorbidities were similar between groups. Sixty-six percent presented with a non-ST-elevation ACS and 54\% underwent CABG. More non-ST-elevation ACS patients underwent $\mathrm{CABG}$ versus PCI (70\% versus 30\%, $<<0.01)$. All patients received ASA, but more CABG patients received 325 versus $80-81$ milligrams $(20 \%$ versus $1 \%, \mathrm{p}<0.01)$. All PCI patients received a P2Y12 inhibitor (primarily ticagrelor) versus $24 \%$ of CABG patients (primarily clopidogrel). All CABG patients received a beta-blocker versus $97 \%$ of PCI patients. Use of ACEI/ARBs was higher in PCI versus CABG patients $(99 \%$ versus $69 \%, \mathrm{p}<0.01)$. Statin use was similar between groups (97\% versus $99 \%, \mathrm{p}=0.45$ ), but more PCI patients received high-dose (91\% versus 57\%, $\mathrm{p}<0.01)$.

Conclusions: Use of ASA and beta-blockers post-ACS was high in both groups. P2Y12 inhibitors and ACEI/ARBs were underutilized in CABG patients even after adjusting for justified non-use, and CABG patients were less likely to receive high-dose statin therapy.

\section{The Utilization of Mineralocorticoid Receptor Antagonists in Patients with Post ST-Elevation Myocardial Infarction Complicated by Left Ventricular Dysfunction}

\author{
Lam $V^{1}$, Patel $P^{2}$, Mohammed $A^{2}$, Vijayaraghavan $R^{2}$, Chin ${ }^{2}$ \\ ${ }^{1}$ University of Toronto, Toronto, $O N$ \\ ${ }^{2}$ Rouge Valley Health System - Centenary Hospital, Toronto, ON
}

Background: The EPHESUS trial demonstrated that early initiation of Mineralocorticoid Receptor Antagonists (MRAs) reduced mortality in post myocardial infarction patients with left ventricular ejection fraction $(\mathrm{LVEF}) \leq 40 \%$ and documented heart failure (HF) or diabetes. Despite mortality benefits, it is hypothesized that MRAs are underutilized in a modern Percutaneous Coronary Intervention (PCI) center.

Objective: This study aims to determine any discrepancies between evidence-based guidelines and current prescribing rates of MRA.

Methods: A retrospective chart review was completed in a primary PCI center on all ST-elevation myocardial infarction (STEMI) patients from January 2016 to December 2016. Inclusion and exclusion criteria for MRA were applied based on those outlined in EPHESUS.

Results: A total of 670 patients presented with a STEMI during the study period. 451 patients did not meet criteria due to length of stay shorter than 3 days. 114 patients did not have a LVEF $\leq 40 \%$. 72 patients had a false positive STEMI, 11 were non-diabetic with no HF symptoms. Ultimately, 22 patients were eligible for MRA therapy. Of those eligible, the mean age was $59,86.3 \%$ were male, median length of stay was 4.5 days, mean LVEF was 35\%, 63.6\% had clinical evidence of HF, $54.5 \%$ were diabetic, and $18.2 \%$ were diabetic and had clinical evidence of HF. $81.8 \%$ with anterior infarct, 36.4\% with inferior infarct, and 9\% had both anterior and inferior infarct. Of the 22 patients eligible, 8 were prescribed MRA therapy. Of the 14 patients not prescribed MRA, 2 were hypotensive while 12 had undocumented reasons.

Conclusion: Despite mortality benefits of MRAs, a care gap exists between evidence-based guidelines and current practice. Reasons for MRA underutilization need to be further studied and addressed. As a result, we are creating pharmacist-implemented initiatives in our practice to raise awareness of MRA indications around healthcare providers and patients.

\section{An Assessment of Modifiable Risk Factor Management in Hospitalized Patients with Type 2 Diabetes Mellitus}

\section{Haines A, KalesnikoffJ, Albers L, Roy C, Semchuk W} Saskatchewan Health Authority, Regina, SK

Background: Treating to targets for glycated hemoglobin (A1C), lowdensity lipoprotein cholesterol (LDL-C) and urine albumin-creatinine ratio (ACR), reduces the risk of micro and macrovascular complications in patients with type 2 diabetes mellitus (T2DM). Evidence from primary care populations shows a persistent gap in the proportion of patients achieving targets and limited evidence is available to demonstrate interventions during hospitalization.

Objectives: Determine the current rate of control of diabetes-related risk factors in an inpatient population with T2DM and identify if target medications are initiated during hospitalization.

Methods: A retrospective assessment of the medical records of patients 40 to 75 years of age with T2DM, taking oral anti-hyperglycemic agents, who had been admitted to hospital with a minimum duration of stay of 72 hours.

Results: Of 193 patients included, A1C, LDL-C, and ACR was obtained in $182 / 193(94.3 \%), 156 / 193(80.8 \%)$, and $121 / 193(62.7 \%)$ and $88 / 182$ (48.4\%), 87/156 (55.8\%), and 52/121 (43.0\%) with available laboratory data achieved targets in the year prior to hospital discharge, respectively. Use of oral anti-hyperglycemic medications, statins and/ or ezetimibe, and angiotensin converting enzyme inhibitors (ACEI) or angiotensin-II receptor blockers (ARB) showed absolute increases of $10.9 \%, 7.7 \%$, and $0.5 \%$ respectively during hospital admission; however, $22 / 69(31.9 \%)$ and 5/69 (7.2\%) were discharged without statins and/or ezetimibe or ACEI/ARB, respectively.

Conclusions: There is room for improvement in the rate of control of diabetes-related risk factors in hospitalized patients with T2DM in acute 
care facilities within Regina Qu'Appelle Health Region. Although hospital practitioners are initiating some patients on target medications during hospitalization, comprehensive multidisciplinary strategies should be implemented to close the treatment gap and align care with evidenced based guidelines to prevent patient morbidity and mortality.

\section{The Antithrombotic Treatment of Patients with Atrial Fibrillation Undergoing Percutaneous Coronary Intervention}

Bezzina $E^{1}$, Bucci $C^{1,2}$, Diamantouros $A^{1,2}$, Radhakrishnan $S^{3,4}$, Ahmed $S^{3,4}$ ${ }^{1}$ Sunnybrook Health Sciences Centre, Department of Pharmacy, Toronto, ON ${ }^{2}$ University of Toronto, Leslie Dan Faculty of Pharmacy, Toronto, ON ${ }^{3}$ Sunnybrook Health Sciences Centre, Division of Cardiology, Toronto, ON ${ }^{4}$ University of Toronto, Faculty of Medicine, Toronto, ON

Background: Determining the optimal antithrombotic regimen for patients with non-valvular atrial fibrillation (NVAF) undergoing percutaneous coronary intervention (PCI) is challenging. Recent literature suggests that dual therapy (DT: oral anticoagulant + P2Y12 inhibitor) may have similar efficacy with less bleeding compared to triple therapy (TT: oral anticoagulant + P2Y12 inhibitor + ASA).

Objective: The main objective of this study was to characterize recent practice patterns in this patient population.

Methods: A retrospective chart review of patients with NVAF who underwent PCI with stenting at a Canadian, academic, tertiary care centre between September 1, 2016 and January 31, 2018 was completed. An electronic survey was sent to cardiologists to better understand prescribing rationale. Study results were reported using basic descriptive statistics including frequencies and percentages, with continuous variables presented as means \pm standard deviations.

Results: Of the 107 patients included in the chart review, 71 (66\%) had an admission diagnosis of acute coronary syndrome. At discharge, 19 patients (18\%) were prescribed TT, $48(45 \%)$ were prescribed DT, and $40(37 \%)$ were prescribed dual antiplatelet therapy (DAPT: ASA + $\mathrm{P} 2 \mathrm{Y} 12$ inhibitor). The decision of whether to anticoagulate was deferred to another hospital in $15 \%$ of DAPT cases. The average planned duration of TT, when prescribed, was 3.9 months. Of the 65 patients discharged directly home, a clear plan, including timeframe, for family doctor and cardiologist follow-up was documented in $54 \%$ and $74 \%$ of cases, respectively. Of 32 cardiologists, 8 (25\%) responded to the survey. All 8 respondents agreed that recent literature has influenced their practice.

Conclusions: Overall, antithrombotic therapy was prescribed by a tailored approach, consistent with the most recent Canadian Antiplatelet Guidelines. The less intense regimen of DT was the most common antithrombotic regimen, illustrating rapid translation of recent literature into clinical practice. This study identified opportunities to improve antithrombotic practice patterns.

\section{A Retrospective, Observational Study of the Management of Patients Hospitalized with Heart Failure with Reduced Ejection Fraction}

Gallagher B, Roy C, Albers L, Krahn K, Semchuk B

Saskatchewan Health Authority, Regina, SK

Background: Guideline directed medical therapy (GDMT) at target doses has been shown to improve survival in heart failure with reduced ejection fraction (HFrEF) and quality indicators exist to measure adherence to guidelines. In Regina, SK, HFrEF is a priority initiative, but it is unknown how patients are being managed.
Objective: To assess practice for alignment with evidence based medication-related recommendations for patients admitted with an exacerbation of HFrEF to an acute care hospital in Regina.

Methods: A retrospective review of inpatient medical records was completed. Patient selection was designed to include only ideal candidates, excluding patients with known contraindication to components of triple medication therapy and whose HFrEF diagnosis was less than six months to allow time to initiate and titrate medications.

Results: Out of 99 patients included, less than half (45.5\%) were prescribed triple medication therapy at discharge, with $82(82.8 \%)$ patients on an angiotensin converting enzyme inhibitor (ACEi), angiotensin II receptor antagonist (ARB) or angiotensin receptor neprilysin inhibitor (ARNI), 93 (93.9\%) on a beta blocker (BB), and $53(53.5 \%)$ on a mineralocorticoid receptor antagonist (MRA). Target dose was achieved for 36/81 (44.4\%) patients on an ACEi, ARB or ARNI, $19 / 92(20.5 \%)$ on a BB, and $3 / 53(5.7 \%)$ on an MRA. Sixty-four $(64.5 \%)$ patients were directed to follow up with their primary care provider, $49(49.5 \%)$ with a cardiologist, and $14(14 \%)$ with the Heart Function Clinic. Over 12 months, 45 (45.4\%) patients were admitted 2 or more times for a HFrEF exacerbation. Thirty-one pharmacist interventions were documented for 18 (18.2\%) patients.

Conclusions: Although many patients may be receiving the benefits of GDMT, there is a treatment gap in the management of patients admitted with HFrEF exacerbations in Regina. Health care providers should take every opportunity to enhance utilization and titration of GDMT to reduce morbidity and mortality.

\section{Evaluation of a Pharmacist-Led Antimicrobial Stewardship Service in a Pediatric Emergency Department}

MacInnis $M^{1,2}$, MacMillan $K^{1,2}$, Fitzpatrick $E^{1,2}$, Hurley $K F^{1,2}$,

MacPhee $S^{1,2}$,Matheson $K^{1,3}$, Black $E^{1,2}$

${ }^{1}$ Dalhousie University, Halifax, NS

${ }^{2} I W K$ Health Centre, Halifax, NS

${ }^{3}$ Nova Scotia Health Authority, Halifax, NS

Background: We implemented a pharmacist-led antimicrobial stewardship (AMS) service for patients discharged from the pediatric emergency department (PED). This service, supported by a collaborative practice agreement, allows pharmacists to follow up with patients and independently stop, start, or adjust antimicrobial agents based on culture results.

Objective(s): The primary objective of our study was to evaluate the impact of this service on the rate of return visits to the PED within 96 hours. The secondary objective was to evaluate the appropriateness of the prescribed antimicrobial agent at follow up.

Methods: This study was completed as a retrospective chart review 6 months pre-implementation (January $1^{\text {st }}, 2016$ to June $31^{\text {st }}, 2016$ ) and 6 months post-implementation (February $1^{\text {st }}, 2017$ to July $31^{\text {st }}, 2017$ ) of a pharmacist-led AMS service. A research assistant extracted data from electronic medical records using a standardized data collection form. All patients discharged from the PED with a suspected infection whose cultures fell within the parameters of the collaborative practice agreement were included in this study. Data were reported descriptively and compared using a two-sided chi-square test.

Results: This study included 1070 patient encounters pre-implementation and 1040 patient encounters post-implementation of the AMS service. The most commonly reviewed culture was urine (38\% pre-implementation and $41 \%$ post-implementation). The rate of return visits to the PED within 96 hours was $12.0 \%$ (129/1070) pre-implementation vs $10.0 \%(100 / 1049), \mathrm{p}=0.07$ post-implementation phase. A significantly 
higher percentage of inappropriate antimicrobial therapy was identified at the time of follow up in the pre-implementation phase $(7.0 \%, 68 / 975)$ compared to the post-implementation phase (5.0\%, 46/952), $\mathrm{p}=0.047$. Conclusion(s): Although this pharmacist-led AMS service did not affect the rate of return visits within 96 hours, it may lead to more judicious use of antimicrobial agents in a PED.

\section{Evaluating the Impact of Prospective Audit and Feedback on the Use of Clindamycin and Quinolones in Clinical Teaching Units}

Karsan $I^{1}$, Elsayed $S^{1,2}$, Popovski $Z^{2}$, Dhami $R^{1,2,3}$

${ }^{1}$ Western University, London, ON

${ }^{2}$ London Health Sciences Centre, London, ON

${ }^{3}$ University of Waterloo, Waterloo, ON

Background: Antimicrobial usage of quinolones and clindamycin was noted to be greater on the general medicine units of a large academic hospital. The Antimicrobial Stewardship Program (ASP) implemented a prospective audit and feedback (PAF) strategy to optimize prescribing of these agents given their propensity to cause Clostridium difficile infection (CDI). In this study, the impact of PAF interventions on quinolone and clindamycin use was evaluated.

Objective: To evaluate the impact of the PAF interventions on the use of clindamycin and quinolones, and the incidence of CDI on clinical teaching units (CTU) at a multi-site tertiary care centre.

Methods: A PAF was introduced in April 2015 at a large academic health centre with two campuses (sites) housing six Clinical Teaching Units for general medicine patients. Using a face-to-face PAF model, medical teams (consisting of a senior medical resident and a pharmacist) to review patients receiving antimicrobials twice weekly. Clindamycin and quinolone utilization were compared using defined daily doses (DDD) per 1000 patient days pre and post-intervention. CDI rates were also monitored.

Results: There was an overall reduction in quinolone use by $68.2 \%$ and $75.4 \%$ in Site 1 and 2, respectively. Clindamycin use decreased in the first year at both sites by $50.5 \%$ and $30.8 \%$, respectively. As the use of both antimicrobials decreased, the corresponding CDI rates at both sites also decreased.

Conclusion: The implementation of PAF intervention has reduced the use of clindamycin and quinolones as well as the incidence of CDI in our centre.

For the table that goes with this abstract, please see Abstract Appendix, available at https://www.cjhp-online.ca/index.php/cjhp/issue/view/188/ showToc

\section{A Retrospective Analysis of the Management of Staphylococcus aureus Bacteremia}

Boucher $A$

Leslie Dan Faculty of Pharmacy, University of Toronto, Toronto, ON

Background: Staphylococcus aureus bacteremia (SAB) is the most common blood infection in Ontario and is associated with a high risk of morbidity and mortality. Adherence to management standards (i.e. echocardiography, appropriate antibiotic therapy, repeat blood cultures, and infectious disease (ID) consultation) have been shown to reduce mortality and recurrence.

Description: The objective of this study was to assess clinical outcomes and adherence to management standards in patients with SAB at an Ontario hospital.
Action: A retrospective chart review was conducted. Patient records were included if the patient had at least 1 positive blood culture for $S$. aureus from 30 August 2015 to 29 August 2016. Patients were excluded if they were aged $<18$, or died, were discharged, or deemed palliative within 2 days of blood culture collection. Collected data included patient demographics, antibiotic treatment, investigations, ID consultation, and clinical outcomes.

Evaluation: Of the 76 SAB patients included in the analysis, 34 (45\%) received echocardiogram, appropriate antibiotic therapy, and repeat blood culture. Transthoracic echocardiography (TTE) and transesophageal echocardiography (TEE) were performed in 64 (84\%) and $19(25 \%)$ patients, respectively. We found that $67(87 \%)$ patients received appropriate empiric and definitive antibiotic therapy, and 41 (54\%) had an appropriate duration of therapy. Repeat blood cultures were performed in $63(83 \%)$ of patient and ID consultation was provided for $33(43 \%)$ of patients. Overall, $10(13 \%)$ patients died and $3(4 \%)$ experienced recurrence within 90 days of a positive blood culture.

Implications: Adherence to each management standard varied. The lowest adherence was associated with TEE, appropriate duration of antibiotic therapy, and ID consultation. Opportunities exists for pharmacist-driven initiatives to improve clinical outcomes and adherence to standards of care in the management of SAB.

\section{Process Measures Associated with a Successful Antimicrobial Stewardship Intervention to Stop a Clostridium difficile Outbreak}

Leung E, Kwee F

Department of Pharmacy, St. Michael's Hospital, Toronto, ON

Background: Antimicrobial Stewardship Programs (ASPs) often use audit and feedback to optimize antimicrobial use for individual patients, which can lead to decreased unnecessary antibiotics and decreased C.difficile. ASP interventions have been described as a successful tool to halt C.difficile outbreaks, but there is limited data on specific process measures.

Description: We describe process measures associated with a successful C.difficile outbreak control strategy (collaboration between ASP and infection control [IC]) on an inpatient, nephrology unit at an urban teaching hospital. C.difficile cases increased from baseline starting November 2015; increased IC was implemented in May 2016. An official outbreak was declared June 2017, and the outbreak team was expanded. ASP Audit and Feedback was implemented May to December 2017.

Action: During ASP implementation, a dedicated ID clinical pharmacist and ID physician rounded with the nephrology multidisciplinary team (pharmacists, nurse practitioner, and nephrology physicians) three times per week to review all antimicrobial prescriptions. The ASP provided advice with attention to avoiding high-risk C.difficile antimicrobials and unnecessary antimicrobials; the outbreak was declared over September $15,2017$.

Evaluation: In total, 151 suggestions were made to the nephrology multidisciplinary team. Results are presented in the Table. Approximately one-quarter of recommendations decreased overall antimicrobial exposure (ie. discontinue, or narrow); one-quarter required escalation of a complicated case to the ID consultation service; and 14\% were nondrug-therapy related (ie. laboratory/diagnostics).

Implications: We have described process measures that were associated with successful cessation of a C.difficile outbreak in a high-risk population at our institution. ASPs can utilize these process measures in future planning of outbreak management strategies. 
For the table that goes with this abstract, please see Abstract Appendix, available at https://www.cjhp-online.ca/index.php/cjhp/issue/view/188/ show Toc

\section{Severe Allergic Reaction Induced by Dexlansoprazole: A Case Report and Literature Review}

Rault $P^{1}$, Poulin $M A^{2}$ Bussières J-F $F^{1}$

${ }^{1}$ CHU Sainte-Justine, Montréal, $Q C$

${ }^{2}$ Université de Laval, Québec, QC

Background: Proton pump inhibitors (PPIs) are one of the most worldwide prescribed drugs and represented twenty-two percent of the medication used in Quebec in 2010. We report a case of hypersensitivity with the Dexlansoprazole, belonging to the new generation of PPIs and marketed in Canada in 2010.

Case Description: A 24-year-old Canadian woman patient underwent an adverse drug reaction related to dexlansoprazole. Fifteen minutes after oral ingestion of $60 \mathrm{mg}$ of dexlansoprazole, she experienced burning sensations in both feet and hands. Then, she suffered from several vomiting, a coalescing maculopapular rash on almost $100 \%$ of her body surface ten seconds after vomiting and a swelling of her face and tongue, as far as partially obstructing her airways. She also passed out twice before her admission to the emergency room. After receiving intraveinous shots of dyphenhydramine, solumedrol, famotidine and ondansetron, she has completely recovered. She was discharged after a 4 hours observation period.

Assessment of Causality: Both the Naranjo (score of eight) and the Koh et $\mathrm{Li}$ (score of nine) scores show a probable imputability of the drug. Skin prick tests with lansoprazole and dexlansoprazole were performed. Only dexlansoprazole prick test was positive. Two months before, our patient had suffered from a primary exposure with the dexlansoprazole who has also led after fifteen minutes to burning hands and feet. This previous reaction was resolved in thirty minutes without any treatment.

Literature Review: We carried out a literature review using Pubmed, CINAHL and Google scholar databases. No case report about hypersensitivity related to dexlansoprazole was found. However, about sixty clinical cases of hypersensitivity were listed with all PPIs. Hypersensitivity with its stereoisomer, Lansoprazole, is one of the most described one.

Importance to Practitioners: To our knowledge, this is the first case report concerning anaphylactic reaction with Dexlansoprazole in the literature.

\section{Environmental Contamination with Nine Antineoplastic Drugs in 79 Canadian Centers}

Hilliquin $D^{1}$, Tanguay $C^{1}$, Gagné $S^{2}$, Caron $N{ }^{2}$, Bussières $J-F^{1,3}$

${ }^{1}$ Unité de recherche en pratique pharmaceutique, Département de pharmacie, CHU Sainte-Justine, Montréal, QC

${ }^{2}$ Centre de Toxicologie du Québec, Institut national de santé publique du Québec, Québec, QC

${ }^{3}$ Faculté de pharmacie, Université de Montréal, Montréal, QC

Background: Antineoplastic drugs traces are measured on many surfaces in healthcare centers. Workers occupationally exposed to these traces are at risk of adverse health effects. In order to reduce their exposure, surface contamination should be kept as low as possible.

Objective(s): To monitor environmental contamination by nine antineoplastic drugs in Canadian centers. To explore the impact of factors that may be associated with surface contamination.
Methods: Twelve standardized sites were sampled in each participating center after a working day, before any cleaning was performed (six in the oncology pharmacy and six in patient care areas). Each sample was prepared to allow quantification of six antineoplastic drugs (cyclophosphamide, ifosfamide, methotrexate, gemcitabine, 5-fluorouracil, irinotecan) by ultra-performance liquid chromatography-tandem mass spectrometry. Three additional antineoplastic drugs were detected, but not quantified (docetaxel, paclitaxel, vinorelbine). The impact of some factors was evaluated with a Kolmogorov-Smirnov test for independent samples.

Results: Seventy-nine Canadian centers participated in 2018. A total of 887 surfaces were sampled, 467 in pharmacy and 420 in patient care areas. Cyclophosphamide was most often measured on surfaces $(32 \%$ positive samples, $75^{\text {th }}$ percentile $=0.0017 \mathrm{ng} / \mathrm{cm}^{2}, 90^{\text {th }}$ percentile $\left.=0.021 \mathrm{ng} / \mathrm{cm}^{2}\right)$. The front grille inside the hood $(81 \%$ of samples positive for at least one antineoplastic drug), the arm rest (79\%), the storage shelf (62\%) and the floor in front of the hood (60\%) were more frequently contaminated. Centers with a higher number of oncology inpatient and outpatient beds, who prepared more antineoplastic drugs per year and used more cyclophosphamide per year had higher concentrations of cyclophosphamide on their surfaces $(\mathrm{p}<0.0001)$.

Conclusion(s): Some working surfaces were frequently contaminated despite the implementation of safe handling guidelines. The use of personal protective equipment remains indisputable. Environmental monitoring can help centers to monitor their practices and identify contaminated areas.

\section{Adverse Effects of High-Dose vs Standard-Dose Dexmedetomidine in the Cardiac-Surgery Population: A Retrospective Cohort Study}

Leung $L^{1}$, Wang $E^{1}$, Lee $B^{2}$

${ }^{1}$ Faculty of Pharmaceutical Sciences, University of British Columbia, Vancouver, $B C$

${ }^{2}$ Faculty of Medicine, University of British Columbia, Vancouver, BC

Background: Dexmedetomidine is a selective alpha- 2 agonist with minimal respiratory depression compared to other sedatives; it is used to facilitate extubation and to treat delirium in the critically-ill. The dexmedetomidine monograph recommends a dose of $0.2-0.7 \mathrm{mcg} / \mathrm{kg} / \mathrm{hr}$ but higher doses have been used in practice. It is not known whether higher-than-recommended doses of dexmedetomidine are safe in cardiac surgery patients. Higher doses have not been studied in this patient population who are potentially at higher risk for adverse effects due to recent myocardium manipulation and hemodynamic instability.

Objective: To determine whether there is an increased risk of hypotension or bradycardia with higher-than-recommended doses of dexmedetomidine in cardiac surgery patients.

Methods: We conducted a retrospective cohort study of patients who received dexmedetomidine for any indication following cardiac surgery at our hospital between 2013 and 2017. The primary outcome was the rate of hypotension or bradycardia for high-dose $(>1.0 \mathrm{mcg} / \mathrm{kg} / \mathrm{hr})$ vs standard-dose $(\leq 1.0 \mathrm{mcg} / \mathrm{kg} / \mathrm{hr})$ dexmedetomidine. Secondary outcomes included individual rates of hypotension, bradycardia, arrhythmias, and hyperglycemia in the high-dose vs standard-dose groups.

Results: Among 245 patients included, 49 received high-dose and 196 received standard-dose dexmedetomidine. Mean doses of dexmedetomidine in the high-dose and standard-dose groups were $0.79 \pm 0.18 \mathrm{mcg} / \mathrm{kg} / \mathrm{hr}$ and $0.45 \pm 0.06 \mathrm{mcg} / \mathrm{kg} / \mathrm{hr}$ respectively. For the primary outcome, $36(73.5 \%)$ patients in the high-dose group and $128(65.3 \%)$ in the standard-dose group experienced hypotension or 
bradycardia $(\mathrm{OR}=1.48, \mathrm{p}=0.340,95 \% \mathrm{CI}: 0.7-3.4)$. In the high-dose group, there were more episodes of transient bradycardia $(<1 \mathrm{hr})$ : $6(12.2 \%)$ vs $2(1.0 \%)$, and more hypotension: $36(73.5 \%)$ vs $128(65.3 \%)$.

Conclusion: This is the first known study of high-dose dexmedetomidine after cardiac surgery. The results may suggest that the rate of hypotension or bradycardia may be slightly higher with high-dose dexmedetomidine compared to standard-dose, but this did not reach statistical significance.

\section{Capturing Medication Safety Culture in Saskatchewan Pharmacies Using the Medication Safety Culture Indicator Matrix}

\author{
Chiu $E^{1,2}$, Sandiford P, Postnikoff L $L^{3}$, Shyshlova $A^{4}, H_{0} C^{1,2,4}$ \\ ${ }^{1}$ Institute for Safe Medication Practices Canada, Toronto, ON \\ ${ }^{2}$ Leslie Dan Faculty of Pharmacy, University of Toronto, Toronto, ON \\ ${ }^{3}$ Saskatchewan College of Pharmacy Professionals, Saskatoon, SK \\ ${ }^{4}$ School of Pharmacy, University of Waterloo, Waterloo, ON
}

Background: Standardized continuous quality improvement (CQI) programs are employed to assist pharmacies in recognizing medication incidents and developing solutions to prevent them. It is important to understand where practice culture surrounding medication safety stands to better support this endeavour.

Description: The objectives of this study were to explore the medication safety culture in Saskatchewan pharmacies and identify whether pharmacies currently held a "blame-and-shame" (i.e. pathological) or "systems-oriented" (i.e. generative) attitude towards safety.

Action: The Medication Safety Culture Indicator Matrix (MedSCIM) tool, developed by the Institute for Safe Medication Practices Canada (ISMP Canada), was used to analyze two sets of medication incidents reported by pharmacies in Saskatchewan from September 2013 to October 2017: one set was associated with patient harm, while another set contained randomly selected incidents from the top three types of medication incidents.

Evaluation: We analyzed 140 harm incidents: $42.8 \%$ of the reports were fully complete, $51.4 \%$ semi-complete, and $5.7 \%$ not complete. Of the 158 randomly selected top three types of incidents, $5.69 \%$ were fully complete, $70.25 \%$ semi-complete, and $24.05 \%$ not complete. Within the harm incidents, $13.5 \%$ implied a "blame-and-shame" culture, $40 \%$ reflected "reactive", $30 \%$ indicated "calculative", and 16.5\% supported a "generative" safety culture. On the other hand, "blame-and-shame", "reactive", "calculative", and "generative" safety culture were represented by $9.49 \%, 85.44 \%, 3.79 \%$, and $1.26 \%$, respectively, of the randomly selected top three types of incidents.

Implications: Our MedSCIM analysis reveals that there is still work to be done to facilitate medication safety culture towards a more "systems-oriented" or "generative" attitude and it appears that pharmacy professionals tend to do so more often if patient harm is involved in the incident. Our study offers a baseline of medication safety culture in Saskatchewan pharmacies as the provincial mandatory standardized CQI program is being rolled out in 2018 .

\section{Analyse descriptive des incidents et accidents médicamenteux de 2011 à 2018 dans un centre hospitalier}

Chabrier A, Atkinson S, Lebel D, Bussieres J-F

CHU Sainte-Justine, Montréal, QC
Contexte : Depuis 2002, un processus structuré de déclaration des incidents et accidents (IA) en établissement de santé a été mis en place au Québec.

Objectif(s) : Décrire et commenter l'évolution des IA médicamenteux (IAM) du $1^{\text {er }}$ avril 2011 au 31 mars 2018 au sein centre hospitalier universitaire.

Méthodologie : Étude descriptive rétrospective. À partir du registre local des IA, nous avons extrait, par année financière, tous les IAM incluant les variables suivantes : gravité (selon NCCMEPR), quart de travail de survenue, catégorie (médication), circonstance, description et la présence d'un médicament à haut risque.

Résultats : Un total de 41350 IA a été déclaré de 2011 à 2018 incluant 12881 IAM (31\%). La répartition des IAM selon la gravité est : A(5\%), $\mathrm{B}(14 \%), \mathrm{C}(67 \%), \mathrm{D}(9 \%), \mathrm{E}(5 \%), \mathrm{F}(\mathrm{n}=9), \mathrm{G}(\mathrm{n}=0), \mathrm{H}(\mathrm{n}=0), \mathrm{I}(\mathrm{n}=2)$. Les IAM surviennent de jour $(36 \%)$, de soir $(31 \%)$ ou de nuit $(33 \%)$. Le top-5 des circonstances identifiées inclut: médicaments non administré (22\%), mauvaise posologie du médicament administré (21\%), mauvais horaire d'administration (10\%), mauvais médicament administré (8\%) et extravasation du médicament $(5 \%)$ et autres $(34 \%)$. Les médicaments à haut risque (MHR) représentent 15\%(1902/12881) des IAM. Le top-10 des MHR représentés inclut: morphine $(\mathrm{n}=497)$, héparine $(\mathrm{n}=262)$, hydromorphone $(\mathrm{n}=216)$, fentanyl $(\mathrm{n}=139)$, kétamine $(\mathrm{n}=124)$, enoxaparine $(n=62)$, milrinone $(n=56)$, méthotrexate $(n=44)$, daltéparine $(\mathrm{n}=41)$, acétaminophène-codéine $(\mathrm{n}=31)$. Le nombre d'IAM déclarés par année diminue à partir du $1^{\text {er }}$ avril 2014.

Conclusion(s) : Les IAM représentent une part importante des IA en établissement de santé. Toutefois la majorité des IAM ne comportent pas de conséquences pour le patient. Les médicaments à haut risque occupent une place importante dans les déclarations des IAM. L'étude ne permet pas d'identifier de causes certaines liées à la diminution du nombre d'IAM déclarés au cours des dernières années.

\section{Comparative Evaluation of Intentional versus Unintentional Medication Discrepancies during Admission Medication Reconciliation}

Abdelaziz H, Levesque T, Smith A, Michèle L, St-Yves J,

Landry-Leblanc S, Lussier S, Savoie M, Gagnon J

Pharmacy Department, Campbellton Regional Hospital,

Vitalité Health Network, Campbellton, NB

Background: During admission medication reconciliation (MedRec), proactive model may include documented intentional medication discrepancies (DIMD), while retroactive model can experience the occurrence of both DIMD and unintentional medication discrepancies (UMD). In the literature, studies comparing and analyzing DIMD and UMD in both such models are scarce.

Objective: The aim of this study was to analyze and quantify both DIMD and UMD in both models of MedRec.

Methods: This comparative analysis included patients previously enrolled in a prospective, observational study that was conducted at Regional Hospital from May to June 2018. The primary end point was to identify the frequency of DIMD and UMD. The secondary end point was to discern the frequency and type of medication classes and the reasons associated with each type of discrepancy.

Results: After reviewing 249 medication reconciliations, 180 patients were enrolled. Of those, 84 patients received the proactive MedRec, while 96 patients received the retroactive model. The total number of medications reconciled was 2118. The percentage of DIMD in the proactive model was significantly more than that in the retroactive model 
(16.3\% vs. $7.3 \%$, respectively; $\mathrm{P}<0.001$ ). In the retroactive model, the UMD were significantly more than the DIMD (25.1\% vs. $7.3 \%$, respectively $\mathrm{P}=0.0003$ ). The most common reason for DIMD was withholding home medication; there was no difference between the retroactive and proactive models $(23.8 \%$ vs $26.9 \% \mathrm{P}=0.59)$. Cardiovascular drugs were the most common class involved in DIMD for both models. The most common UMD was omission (78.8\%) and the most common class was gastrointestinal agents in the retroactive model.

Conclusion: This study demonstrated that the proactive model had a positive impact on reducing the medication errors associated with late DIMD or UMD.

\section{Transition of an Independent Website for a Professional Association Branch to a Microsite Integrated within the Website of the Parent Organization}

Crawley $A^{1}$, Dumont $Z^{2}$, MacLeod $K^{2}$, Myers ${ }^{2}$, Regier $A^{3}$, Tang $A^{3}$, Tangedal $K^{2}$

${ }^{1}$ Saskatchwan Health Authority, Prince Albert, SK

${ }^{2}$ Saskatchewan Health Authority, Regina, SK

${ }^{3}$ Saskatchewan Health Authority, Saskatoon, SK

Background: The Saskatchewan branch of the Canadian Society of Hospital Pharmacists (CSHP) independently operated a website for communication with local members. Functions included: advertising events, awards, residency programs; newsletter distribution; sharing policies, procedures, contact information, including forwarding email addresses; members-only access to meeting minutes and resources. CSHP presented the branch with the opportunity to develop a subsection, or "microsite", within its website. Benefits included sharing resources and lowering expenses; known challenges to microsite operation included loss of autonomy, due to need for a centralized website administrator. The branch elected to exercise the option. A transition working group was formed.

Description: The unique situation presented a rare opening to review existing website functions and content prior to migration. An engagement strategy was used to inform development.

Action: Two online surveys were conducted. A volunteer survey was used to determine communication needs of branch council members. A branch member survey was used to determine uses for a branch website. Findings aided in development of council contact mechanisms (eg, email addresses), site navigation, and determining which content would be migrated, eliminated, or would need to be created. Progress was periodically shared with branch council for feedback and guidance in navigating obstacles.

Evaluation: Thirteen of 19 branch council members responded to the first survey. Branch email addresses were rarely used by council members. Only three positions required such addresses: president, communications chair, and fundraising coordinator. A fourth address was eventually added to facilitate award nominations. An address naming convention was established. Forty-seven responses (approximately 20\% of branch members), were received from the second survey. The main reasons reported for using the website included events, awards, and contact information, and to read branch news.

Implications: The information gathered increased the likelihood that the new website's function and content aligns with the needs of branch council and members.
Opioid Stewardship: Implementing Proactive, Pharmacist-Led Reviews for Patients Co-Prescribed Opioids and Benzodiazepines at an Urban Academic Family Health Team

Tilli $T^{1,2}$, Kiran T,4,5, Dewhurst $N^{1,2}$, Hunchuck J,2,3,4

${ }^{1}$ St. Michael's Hospital, Toronto, ON

${ }^{2}$ Leslie Dan Faculty of Pharmacy, Toronto, ON

${ }^{3}$ Department of Family and Community Medicine, St. Michael's Hospital, Toronto, $O N$

${ }^{4}$ Department of Family and Community Medicine, University of Toronto, Toronto, $O N$

${ }^{5}$ Centre for Urban Health Solutions, Li Ka Shing Knowledge Institute, Toronto, ON

Background: In 2017, almost 4,000 Canadians died from opioid-related causes. Co-administration of opioids and benzodiazepines is a risk factor for overdose. Primary care pharmacists, functioning as opioid stewards, could address co-prescribing; however, implementation has not been characterized.

Objective: To implement proactive, pharmacist-led reviews of patients with chronic non-cancer pain co-prescribed opioid(s) and benzodiazepine(s).

Methods: A quality improvement approach, utilizing Plan-Do-StudyAct methodology, was employed from November 2017 to May 2018. Four Plan-Do-Study-Act cycles were conducted across two academic family health team sites associated with a tertiary care medical centre in Toronto. The intervention consisted of a pharmacist: (1) identifying patients through medical record queries; (2) developing care plans; (3) discussing recommendations with prescribers; and (4) discussing implementation with patients. The intervention was refined, according to prescriber and patient interviews, to have the pharmacist: (1) engage with physicians in-person; (2) review all a physician's co-prescribed patients with them in a single meeting; (3) increase their visibility; and (4) provide education on pharmacists' scope of practice. Outcome, process, and balancing measures were collected monthly.

Results: Thirty-five patients (100\%) were assessed by the pharmacist. There was an increase in the number of patients with pharmacist involvement in their pain management from $5(14 \%)$ at baseline to $23(66 \%)$ post-intervention. Patients offered an opioid taper increased from $6(17 \%)$ to $10(29 \%)$ and those with an active taper increased from $2(6 \%)$ to $8(23 \%)$. Mean total daily opioid dose decreased $11 \%$ from 50.5 milligrams morphine equivalent (MME) to $44.7 \mathrm{MME}$. Mean total daily benzodiazepine dose decreased $8 \%$ from 9.9 milligrams diazepam equivalent (MDE) to 9.3 MDE.

Conclusion: The implementation of proactive, pharmacist-led reviews for co-prescribed patients was accepted. A new role for primary care pharmacists as opioid stewards exists. Future studies could expand to additional sites and patients with other risk factors for overdose.

\section{Optimizing Patient Education of Oncology Medications: A Quantitative Analysis of the Patient Perspective}

Kaupp $K^{1}$, Scott $S^{1}$, Minard $L^{1}$, Lambourne $T^{2}$

${ }^{1}$ Department of Pharmacy, Nova Scotia Health Authority (Central Zone), QEII Health Sciences Centre, Halifax, NS

${ }^{2}$ Department of Pharmacy, Nova Scotia Health Authority (Northern Zone), Colchester East Hants Health Centre, Truro, NS

Background: With the ever-increasing complexity of cancer treatments, oncology medication patient education is becoming a progressively important component of cancer care. Education increases patients' 
abilities to make informed decisions, increases medication adherence, decreases feelings of anxiety and depression, improves patient satisfaction, and allows patients to feel a sense of control. Despite this, cancer patients frequently report that they receive inadequate information and feel that their education needs have not been met.

Objective: To explore patients' perspectives of optimal oncology medication education across Nova Scotia.

Methods: A descriptive survey of adult oncology outpatients. Participants included adult medical, hematological and gynaecological oncology outpatients receiving intravenous chemotherapy within Nova Scotia Health Authority between January 26 and April 30, 2018.

Results: One hundred forty-two responses were included. Forty-one percent and $47 \%$ of respondents reported being satisfied or very satisfied with their oncology medication education, respectively. Thirty percent and $43 \%$ of respondents would like the opportunity to receive education or follow-up from a hospital pharmacist, respectively. Respondents with post-secondary education were found to have 2.82 higher odds of wanting to make an appointment for education with a hospital pharmacist. Respondents with a hematological malignancy were found to have 9.23 higher odds of receiving education from a hospital pharmacist.

Conclusions: Patients were generally satisfied with their oncology medication education despite the majority not receiving education from a hospital pharmacist. Patients with a higher level of formal education were more likely to want the opportunity to schedule an appointment for education with and/or receive follow-up from a hospital pharmacist. The oncology medication education participants received in the past appears to align with their education preferences. Findings from this research can be used to optimize the limited time healthcare professionals have to provide meaningful and effective oncology medication patient education and improve patient-centered care.

\section{Development of Candidate Choosing Wisely Recommendations for a Professional Society}

Barry $A^{1}$, Dumont $Z^{2}$, Lyder $C^{3}$, Saulnier $L^{4}$

${ }^{1}$ Lower Mainland Pharmacy Services, Chilliwack, BC

${ }^{2}$ Saskatchewan Health Authority, Regina, SK

${ }^{3}$ CSHP, Edmonton, $A B$

${ }^{4}$ Horizon Health Network, Moncton, NB

Background: Choosing Wisely Canada (CWC) is a campaign to help patients and clinicians make care choices. CWC partners with professional societies to develop recommendations regarding potentially unnecessary tests and treatments: those not supported by evidence and could result in harm to patients.

Description: The Excellence Initiative Steering Committee of the Canadian Society of Hospital Pharmacists (CSHP) undertook a campaign to develop CWC recommendations that represent the unique voice of pharmacists from hospitals and related health care settings.

Action: A working group was formed. An initial list of recommendations was elicited from members via promotion at CSHP conferences and through an online survey. Suggested recommendations were compiled and duplicates were removed or combined. The remaining were sorted into two categories: 'medication-based' (recommendations), those specific to a medication or group; 'practice-based', those related to general principles of medication therapy. A second survey asked members to rank their 'top five' from each category, resulting in a short-list of contenders. Evidence supporting each was curated. Comparing supporting literature and considering potential for overlap or redundancy (with self and other professional societies) resulted in contenders being removed or combined to make the final list of candidate recommendations. These were presented to the CSHP board for approval before submission to the CWC publication process.

Evaluation: The first member survey resulted in 184 suggested recommendations from 65 members. Seventeen medication-based and 14 practice-based recommendations were re-circulated to membership through the second survey, garnering responses from 263 members. Eleven recommendations distinctly represented member input. Seven recommendations, with accompanying supporting literature, were presented to the board.

Implications: Developing the list of candidate recommendations was an opportunity to engage all members in an initiative to improve patient care. The CWC campaign provides a rare platform for professionals to share with each other, the public at large, and the patients for whom they provide care.

\section{The Impact of Delisting Docusate from a Hospital Formulary}

McGuire $A^{1}$, Wyllie $A^{1}$, Fan-Lun $C^{1,2}$, Mantas $I^{1}$

${ }^{1}$ Pharmacy Department, Mount Sinai Hospital, Sinai Health System, Toronto, ON

${ }^{2}$ Leslie Dan Faculty of Pharmacy, University of Toronto, Toronto, ON

Background: Docusate is frequently prescribed to manage constipation despite being no more effective than placebo. Docusate contributes to polypharmacy and medication costs, increases healthcare provider workload and delays initiation of effective treatments. Consequences of inadequately treated constipation include fecal impaction and bowel obstructions. This study examines the impact of delisting docusate from the formulary at our institution in November 2015.

Objectives: To determine change in volume of dispensed doses of laxatives in the year following the delisting of docusate. Impact on acquisition costs of other laxatives and incidence of negative sequelae of constipation were secondary objectives.

Methods: Statistical process control methods were applied to examine dispensing patterns of bowel medications in the years preceding and following the intervention of delisting of docusate from the formulary. Control charts were created using monthly dispensed volumes of sennosides, lactulose, polyethylene glycol, magnesium hydroxide, bisacodyl and glycerin suppositories. Significant changes were defined as data exceeding upper and lower control limits. Monthly acquisition drug costs and incidence of bowel obstructions using ICD-codes were retrieved, tabulated and analyzed for change before and after the intervention.

Results: In the year following delisting of docusate, monthly dispensed volumes of each bowel medication were within the upper control limits of the XmR control charts, indicating no significant increase in use. Prior to the intervention, 244 combined intestinal obstructions and impactions were recorded. This decreased to 210 in the year following the delisting. In the year preceding delisting, $\$ 5,075$ was spent on docusate. Total costs for bowel medications of interest before and after the intervention were $\$ 23,969$ and $\$ 17,674$, respectively.

Conclusions: This study supports removing docusate from hospital formularies. In the year following delisting, no significant increases in dispensing volumes of bowel medications were observed. This intervention did not increase drug costs or rates of bowel obstruction at our institution. 


\section{Pharmacist Review of Computer Physician Medication Order Entry in Hospitals: A Prospective Observational Study of Pharmacist Interventions}

Prech P, Newman P

NorthWest Telepharmacy Solutions, Winnipeg, MB

Background: Medication safety standards for hospitals require pharmacist review of all medication orders (MO) before the first dose is administered. With the advent of computer-based physician order entry systems (CPOE) embedded with clinical decision support software, the need for pharmacist MO review is occasionally questioned.

Description: This prospective observational study involved telepharmacist review of CPOE from $2300-0700 \mathrm{H}$ in a group of 12 specialty and community hospitals (28-403 beds) utilizing 24 hour telepharmacy services over 10 months, 3 years following the introduction of a shared CPOE system with clinical decision support.

Action: During pharmacist CPOE review, MOs requiring intervention by the pharmacist and the reason for the intervention was recorded.

Evaluation: A total of 40,982 CPOE MO's were reviewed for accuracy and appropriateness by the telepharmacist. Of these, 5287 (12.9\%) had a problem identified that required pharmacist intervention (correction). Of the MOs requiring intervention, the most common was missing or incorrect assignment of the medication to a specific drug product or dosage form, 2319 (44\%) orders. In 1015 cases (19\%), the MO was prescribed at the incorrect time or frequency. For 684 orders (13\%), the automatic therapeutic substitution of a $\mathrm{MO}$ to a medication on the hospital formulary list was unsuccessful. Duplicate MOs totaled 531 $(10 \%)$. The dose or dose unit was incorrect in 498 cases (9\%). A placeholder intended for a drug not contained in the system was used inappropriately when the prescribed drug was available in the system in 240 cases (5\%).

Implications: Even in hospital settings where a CPOE system with clinical decision support has been well established, a significant number of MOs show deficiencies. Review of CPOE by a pharmacist before the first dose of a medication is administered is an essential requirement to ensure the safety of drug therapy in hospitals.

\section{Determining Patient and Caregiver Values and Needs from an Outpatient Oral Anticancer Therapy Program: A Qualitative Needs Assessment \\ Selvathilagan $K^{1,2}$, Rambout $L^{1}$, Wentzell $J^{1}$, Robinson $J^{1}, N g L^{1}$, Richler $M^{1}$ \\ ${ }^{1}$ Department of Pharmacy, The Ottawa Hospital, Ottawa, ON ${ }^{2}$ Leslie Dan Faculty of Pharmacy, University of Toronto, Toronto, ON}

Background: Anticancer treatment is increasingly shifting towards orally administered medications. As treatment moves out of cancer centres and into patients' homes, medications will need to be managed more independently. However, health authorities have identified that patient supports for managing take home anticancer treatment are currently inadequate. In response, many cancer centres have developed oral anticancer therapy programs to improve care. A needs assessment of this patient population is warranted to inform foundational components of an effective oral anticancer therapy program.

Objective: To determine patient and caregiver values and needs from an oral anticancer therapy program.

Methods: A single-centre, qualitative, descriptive study was conducted in an outpatient cancer centre at a tertiary care hospital between March 19 and May 18, 2018. Adult patients receiving oral anticancer therapy for a malignant indication were eligible for recruitment. Data were collected through semi-structured interviews that were audio-recorded and transcribed. Transcripts were analyzed using conventional content analysis to generate codes and establish prominent themes.

Results: Twelve interviews were conducted (mean age 66 years) and a total of nine different oral agents were captured. Four major themes were identified: 1) methods of information and education delivery, 2) drug access concerns, 3) baseline medication concerns; and 4) value of convenient and collaborative cancer care.

Conclusion: Patients desired medication information, particularly concerning efficacy and common side effects, to be delivered in written format. Delays in access to drug therapy were a frequent source of concern. Lastly, convenient and seamless continuity in care is valued and enhances the overall experience with the cancer care system. Results from this study will be used to guide quality improvement initiatives within our institution's oral anticancer therapy program.

\section{Telepharmacist Medication Order Review: A Prospective Observational Study in Healthcare Systems}

Newman P, McDonald K, Dhaliwall S, Rubland L, Adams C NorthWest Telepharmacy Solutions, Deep River, ON

Background: Up to $15 \%$ of medication orders have a prescribing error ( $\mathrm{RxE}$ ), affecting $1 / 3$ of patients during their hospital admission. RxEs cause significant health and economic consequences: increased health-care utilization, morbidity and mortality. A successful intervention to reduce $\mathrm{RxEs}$ is pharmacist medication order review (PMOR) prior to the administration of the first dose. Despite RxE frequency and improved patient safety with PMOR, hospital pharmacy hours in Canada average $79 \mathrm{~h} /$ week ( $47 \%$ of the time). Moreover, in small hospitals, PMOR occurs in $60 \%$ of prescriptions prior to administration of the first dose. In the US, $79 \%$ of hospitals have $24 / 7$ PMOR, 20\% supported by telepharmacy. Telepharmacist services have demonstrated a positive impact on PMOR, patient safety, and healthcare costs. Data on telepharmacy PMOR in Canada are lacking.

Description: This 28-day prospective observational study included 47 pharmacists performing PMOR in 25 health care sites, ranging from 18-389 beds utilizing 24-hour telepharmacy services.

Action: During PMOR telepharmacists documented: facility, software system, type of PMOR (pharmacist order entry or, verification of technician, RN or prescriber entry), time block, total number of orders reviewed and time required for PMOR. Categorical and continuous data were described using descriptive statistics and tests for association.

Evaluation: Pharmacist performed PMOR for 1095 orders in a median time of $1.3 \mathrm{~min} /$ order. Meditech ${ }^{\circledR}$ (Client 35\%, DOS 24\%) was the most common software system. Monday-Friday accounted for the majority of PMOR $(20,17,21,17$, and $15 \%$ respectively). The busiest time block was 8 am-12pm (39\%). Significant differences were found in time/order for: time blocks (daytime vs evening/night/weekends, daytime), software systems, and PMOR (pharmacist order entry vs. verification and entry verification technician, $R N$, prescriber).

Implications: Data on telepharmacy services will allow both Telepharmacy providers and healthcare leadership to make informed decisions on PMOR around the clock to improve patient outcomes. 


\section{Patients' Perspectives on a Self-Administration of Medication Program in a Rehabilitation Hospital}

Neville $H^{1}$, Sehoal $C^{1,2}$, MacMillan $K^{1,2}$, Kooka $C^{1,2}$, Haire $M^{1}$, Harding $C^{1}$, MacFarlane-Conrad $M^{1,3}$, MacKay $S^{1}$, Mountain $A^{1,3}$, Nodwell $L^{1}$, Short $C^{1,3}$, White Jankari $K^{1}$

${ }^{1}$ Nova Scotia Health Authority, NS

${ }^{2}$ College of Pharmacy, Dalhousie University, Halifax, NS

${ }^{3}$ Department of Medicine, Dalhousie University, Halifax, NS

Background: A self-administration of medication program (SMP) is designed to help patients learn to manage their own medications before discharge to the community. Previous studies on patient satisfaction with SMP reported mostly positive responses, but also some mixed findings.

Objective: To determine patients' opinions of a medication self-administration program in an inpatient rehabilitation setting.

Methods: Inpatients enrolled in an SMP in a rehabilitation hospital were invited to participate in a research study to evaluate patients' capacity to manage their medications. Following discharge, participants took part in a one month and six month follow-up telephone call to confirm medication regimens and adherence. A patient satisfaction survey was administered during the one month follow-up. Survey data were entered in a Microsoft Access database and summarized descriptively.

Results: There were 90 participants who consented to the study and met inclusion criteria. Patient satisfaction surveys were administered from January 6, 2017 to May 13, 2018, and 87 participants responded to the survey. Responses indicated that $97 \%$ of participants liked taking their own medications. The most common reasons reported were that the patients liked being independent, having control over their medications, and the SMP helped them understand why they were taking them. Other themes were helping nursing staff who were busy and establishing a medication-taking routine. Overall satisfaction rating indicated that $93 \%$ of survey participants were satisfied with the SMP; $6 \%$ felt neutral; and $1 \%$ felt somewhat dissatisfied. Patients who were dissatisfied noted that they preferred someone else administer their medications.

Conclusions: Patient feedback on the SMP was overall positive, and the ability to administer their own medications was valued. Survey data will be used to help determine the patient population who will most likely benefit from SMP and to improve program quality.

\section{Clinical Burden of Antibiotic Resistance Following Implementation of a Multidisciplinary Antimicrobial Stewardship Initiative in a Major Tertiary Care Center: A Controlled Interrupted Time Series Analysis over 14 Years}

Peragine $C^{1,2}$, Walker $S A N^{1,2,3,4^{*}}$, Leis $J A^{3,4,5,6,7}$, Simor $A E^{3,4,5,6}$ ${ }^{1}$ Department of Pharmacy, Sunnybrook Health Sciences Centre, Toronto, ON ${ }^{2}$ Leslie L. Dan Faculty of Pharmacy, University of Toronto, Toronto, ON ${ }^{3}$ Division of Infectious Diseases, Sunnybrook Health Sciences Centre, Toronto, $O N$

${ }^{4}$ Sunnybrook Health Sciences Centre Research Institute, Sunnybrook Health Sciences Centre, Toronto, ON

${ }^{5}$ Faculty of Medicine, University of Toronto, Toronto, $O N$

${ }^{6}$ Division of Infectious Diseases, Department of Medicine, Toronto, ON

${ }^{7}$ Department of Infection Prevention and Control, Toronto, ON

Underlined Author: Pharmacy Graduate Student (PhD) working under supervision of Sandra Walker at time of study (Presenter)

* Senior Author, now retired, affiliations at time of study completion; sequence determines credit approach to authorship
Background: Reducing antimicrobial resistance (AMR) is a major incentive for institutional antimicrobial stewardship programs (ASPs). However, there remains a paucity of high quality data evaluating the impact of these programs on nosocomial AMR. The Sunnybrook Health Sciences Centre (SHSC) Bayview Campus is a shared site, home to a 627-bed acute care hospital and a 530-bed long-term-care facility (LTCF). A prospective audit-and-feedback (PAF) ASP was implemented in the acute care facility in October 2009. No specific intervention was initiated in LTCF, but there was potential for antimicrobial prescribing in the LTCF to be influenced by policy and practice at the adjoining acute care facility.

Objectives: The objective of this study was to evaluate the impact of the SHSC PAF-ASP on the burden of antibiotic-resistant organisms (ARO) and multidrug-resistant organisms (MDRO) and on inpatient antimicrobial (AMU) in the 7 years following program implementation.

Methods: Patient-level microbiologic and AMU data were retrospectively obtained over a 14-year study period (October 2002-September 2016). Interrupted time series Poisson regression models were used to detect PAF-ASP associated changes in the incidence and trend of hospitalacquired (HA-) ARO, HA-MDRO, and targeted (TGD) AMU and infer program impact. Changes in community-acquired (CA-) and long-term-care facility-acquired (LTCF-) ARO and MDRO were assessed for comparison.

Results: PAF-ASP implementation was associated with improvements in HA-ARO incidence $(9.3 \%$ reduction/post-intervention period, $\mathrm{p}<0.0278), \mathrm{HA}-\mathrm{MDRO}$ incidence (12.6\% reduction/post-intervention period, $\mathrm{p}=0.1319)$, and the trends of both outcomes. Improvement in TGD AMU, increases in CA-ARO and CA-MDRO incidence, and attenuated effects on LTCF-ARO and LTCF-MDRO incidence were found.

Conclusions: Implementation of the acute care PAF-ASP was associated with improvements in acute care AMR and AMU. The absence of improvement in corresponding CA-AMR outcomes, and limited improvement in the corresponding LTCF AMR outcomes, strengthen the causal inference of the acute care PAF-ASP curbing development of acute care AMR.

\section{Ceftobiprole plus Vancomycin for the Treatment of Methicillin-Resistant Staphylococcus Aureus Infective Endocarditis: Case Report}

Curran $J^{1}$, Silverman $M^{1,2}$, Elsayed $S^{1,2}$, Dhami $R^{1,3}$

${ }^{1}$ London Health Sciences Center, London, ON

${ }^{2}$ University of Western Ontario, London, $O N$

${ }^{3}$ University of Waterloo, Waterloo, $\mathrm{ON}$

Background: Ceftobiprole is a cephalosporin antibiotic with broad coverage of gram-positive and gram-negative bacteria, including Methicillin-Resistant Staphylococcus Aureus (MRSA). It is indicated for hospital-associated and community-acquired pneumonia with ongoing trials exploring its efficacy in soft-skin tissue infections as well as MRSA bacteremia. We report a case of MRSA bacteremia secondary to infective endocarditis, successfully cleared with ceftobiprole and vancomycin combination therapy.

Case Description: A 75-year-old male presented to hospital with sepsis secondary to MRSA bacteremia. He was initially treated with vancomycin; however, repeat blood cultures were persistently positive for MRSA. Therapy was switched to a combination of cloxacillin and daptomycin. Despite modest clinical improvement, blood cultures remained positive. A transesophageal echocardiogram confirmed implantable cardiac defibrillator (ICD) infective endocarditis, without 
vegetation. Because the patient remained bacteremic and had developed eosinophilic pneumonitis secondary to daptomycin, antibiotic therapy was changed to ceftobiprole and vancomycin. He clinically improved and blood cultures returned negative. The patient received 6 days of ceftobiprole, after which the ICD was extracted. Post ICD removal, therapy was changed to vancomycin, gentamicin, and rifampin for 6 weeks.

Assessment of Causality: During ceftobiprole therapy, the patient clinically improved. Positive blood cultures returned and remained negative. Vancomycin was unable to clear the bacteremia despite known sensitivity and therapeutic concentrations. As per the WHO-UMC causality system criteria, it is probable that ceftobiprole contributed to the microbiological cure of MRSA bacteremia.

Literature Review: Animal models have demonstrated synergy with vancomycin and ceftobiprole for infective endocarditis, as well as superiority as monotherapy for MRSA infection compared to vancomycin, daptomycin and linezolid. There is 1 published case report of clinical success with ceftobiprole as salvage therapy for prosthetic valve endocarditis.

Importance to Practitioners: Ceftobiprole provides a safe treatment alternative for MRSA infective endocarditis.

\section{Antimicrobial Guideline Concordance is Low in Cancer Patients with Febrile Neutropenia Admitted to General Internal Medicine at an Academic Hospital}

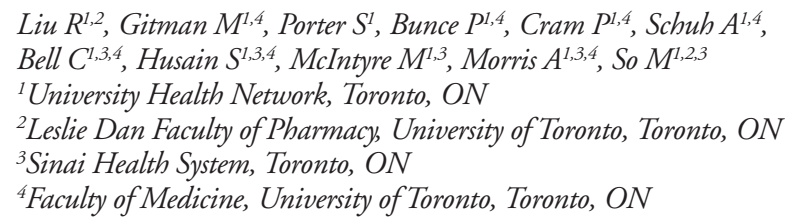

Background: Febrile neutropenia (FN) is a medical emergency which can lead to significant morbidity and mortality for oncology patients. Institution-specific guidelines for febrile neutropenia (FN) have been recommended as an antimicrobial stewardship intervention in oncology patients to reduce unnecessary antibiotic use without adverse outcomes. Institution guideline specified empiric antimicrobials are piperacillin/ tazobactam plus aminoglycoside in high-risk FN patients, and cefazolin plus aminoglycoside in low-risk FN patients. Meropenem is an accepted alternative for patients with self-reported penicillin allergies.

Objective: To evaluate guideline concordance and antimicrobial management in oncology patients admitted to the General Internal Medicine (GIM) unit at an academic hospital.

Methods: A retrospective observational study was conducted in patients admitted with FN from July 1, 2016 to June 30, 2017. Patients were classified as either low-risk or high-risk according to cancer diagnosis and chemotherapy received. The proportion of patients administered guideline-specified empiric antimicrobials within 48 hours of admission to GIM was the primary outcome. Secondary outcomes were proportion of patients whose empiric antimicrobials were active against pathogens isolated, rate of antimicrobial adverse events, and 30-day readmissions.

Results: One hundred patients were included, of which 34\% (34/100) were low-risk FN and 66\% (66/100) as high-risk FN. Antimicrobial management was guideline-concordant in 59\% (59/100) of all admissions. In the low-risk group, guideline concordance was $35 \%$ (12/35), and in the high-risk group, 71\% (47/66). Source of infection was identified in 50\% (50/100), and empiric antimicrobials were active against $94 \%(17 / 18)$ of the pathogens isolated. Average length of stay was $6.6 \pm 3.9$ days, and 30-day readmission rate was 23\% (23/100). Antimicrobial adverse events occurred in 16\% (16/100) of admissions.
Conclusion: Guideline concordance in the antimicrobial management of FN patients is low in GIM at 59\%. Future qualitative studies to identify factors influencing antimicrobial prescribing behaviours to improve knowledge translation are warranted.

\section{Drug Utilization Evaluation of Ceftolozane/ Tazobactam in a Canadian Academic Teaching Hospital System}

Romanowski $A^{1,2}$, Hamandi $B^{1,2}$, Murdoch F $^{2}$ Wong $G^{1,2}$

${ }^{1}$ University of Toronto, Toronto, $O N$

${ }^{2}$ University Health Network, Toronto, ON

Background: Ceftolozane/Tazobactam (tol/taz) is a combination of a 3rd generation cephalosporin with beta-lactamase inhibitor licensed in Canada for treatment of complicated intraabdominal infections and complicated urinary tract infections. The efficacy of tol/taz against Pseudomonas aeruginosa has been demonstrated in vitro and in phase 3 trials. Phase 3 data for nosocomial pneumonia indications is pending. Understanding the role of tol/taz with respect to antimicrobial stewardship principles will be important.

Objective: To carry out a drug utilization evaluation of tol/taz in a Canadian academic teaching hospital system.

Methods: A pharmacy inventory usage report for 1 year from August 2017 to 2018 identified patients who received tol/taz. Patient electronic medical records were accessed to determine: indication/site of infection, dose, duration, causal organism, susceptibility, co-administered antibiotics, unit/ward, patient immune status, microbiological cure, hospital mortality, $C$. difficile infection, renal function.

Results: For the table that goes with this abstract, please see Abstract Appendix, available at https://www.cjhp-online.ca/index.php/cjhp/issue/ view/188/showToc

Notable traits in the patient population receiving tol/taz were immunosuppression, renal dysfunction, and a high level of acuity, as indicated by the unit of patient admission. We used tol/taz for 11 patients with pleural infections, and 3 with complicated intra-abdominal infection. Microbiological cure was low at $19 \%$, however hospital survival at 30 days was $88 \%$.

Conclusion: Tol/Taz may be considered for formulary addition as an alternative agent when primary and secondary therapies have failed or resistance to these agents has been detected and documented. The use of tol/taz in our study was appropriate most of the time from an antimicrobial stewardship perspective. Factors limiting use include: development of $P$. aeruginosa resistance, lack of clinical data in patients with multiple comorbidities or renal dysfunction, cost, and lack of licensed indications for pleural infections.

\section{A Retrospective Case Series Assessing Ceftolozane/Tazobactam Use at a Large Academic Centre}

Xu $A^{1}$, Elsayed $S^{1,2}$, Dhami $R^{1,3}$

${ }^{1}$ London Health Sciences Centre, London, ON

${ }^{2}$ Western University, London, $O N$

${ }^{3}$ University of Waterloo, Waterloo, ON

Background: Ceftolozane/tazobactam is a novel cephalosporin and beta-lactamase inhibitor combination approved for the treatment of complicated urinary tract infections (cUTI) and complicated intraabdominal infections (cIAI). It demonstrates activity against some strains of multi-drug resistant Pseudomonas aeruginosa (MDR-PS); however, 
there is limited clinical experience in off-label indications such as pneumonia (PNA) or osteomyelitis (OM). Pharmacokinetic literature suggests that higher doses may be required for tissue penetration particularly in the lungs.

Objectives: The purpose of this retrospective study is to describe a case series of ceftolozane-tazobactam treatment and evaluate the clinical and microbiologic outcomes at a multi-site tertiary care institution.

Methods: A retrospective chart review was conducted on patients who were administered ceftolozane/tazobactam from June 2016 to May 2018. A predefined data collection form was used to gather patient, infection and treatment-related information. Data collection was summarized using descriptive statistics. Clinical failure was defined as mortality, discontinuation of drug or retreatment of index infection.

Results: Over 24 months, 9 patients received ceftolozane-tazobactam. Mean age was 55 and average Charlson Comorbidity Index was 13. The most frequently isolated pathogen was MDR-PS (8/9). Of the 5 patients with PNA, 3 patients experienced clinical failure, 1 was indeterminate, and 1 successfully cleared the infection with a higher dosing regimen. Of the 2 patients with cIAIs, both experienced clinical and microbiologic failure. The 2 patients with OM experienced both clinical and microbiologic success.

Conclusion: Ceftolozane/tazobactam was used for off-label indications predominantly in respiratory and bone infections where MDR-PS coverage was required. Further studies are needed to support optimization of indication based dosing.

\section{Using Monte Carlo Simulation to Evaluate Tigecycline Dosing Strategies for Bacteria with Raised Minimum Inhibitory Concentrations in Non-Critically III Patients}

\author{
Kispal B ${ }^{1,2}$, Walker $S A N^{1,2,3,4^{*}}$ \\ ${ }^{1}$ Sunnybrook Health Sciences Centre, Department of Pharmacy, Toronto, ON \\ ${ }^{2}$ University of Toronto, Leslie L. Dan Faculty of Pharmacy, Toronto, ON \\ ${ }^{3}$ Sunnybrook Health Sciences Centre, Division of Infectious Diseases, \\ Toronto, $O N$ \\ ${ }^{4}$ Sunnybrook Health Sciences Centre Research Institute, Toronto, ON \\ Underlined Author: Pharmacy student working under supervision of \\ Sandra Walker at time of study (Presenter) \\ * Senior Author, now retired, affiliations at time of study completion
}

Background: Tigecycline is one of the few antibiotic options that exist for treating multi-drug resistant (MDR) gram-negative bacteria.

Objective: The objective was to use Monte Carlo Simulation (MCS) to determine if safe tigecycline dosing options that attained breakpoints for pharmacokinetic-pharmacodynamic (PK-PD) targets in non-critically ill patients could be identified.

Methods: Published studies were included if they evaluated clinically relevant tigecycline dosing regimens and provided mean $\mathrm{PK}$ variables of interest (at minimum 2 of: elimination rate constant $\left(\mathrm{k}^{-1}\right)$ or half-life $\left(\mathrm{t}_{1 / 2}\right)$, and volume of distribution $\left(\mathrm{V}_{\mathrm{d}}\right)$ or clearance $(\mathrm{CL})$ ), with corresponding standard deviations (SD). Weighted mean and weighted SDs for each PK parameter were determined. Food and Drug Administration (FDA) minimum inhibitory concentration (MIC) tigecycline breakpoints for susceptible (MIC $\leq 2 \mu \mathrm{g} / \mathrm{mL}$ ), intermediate (MIC $4 \mu \mathrm{g} / \mathrm{mL}$ ), and resistant (MIC $\geq 8 \mu \mathrm{g} / \mathrm{mL}$ ) Enterobacteriaceae were used. MCS probability distributions (1 million iterations) for PK-PD target attainment of area under the curve for total tigecycline plasma concentration from 0 to $24 \mathrm{~h}$ following an intravenous dose $\left(\mathrm{AUC}_{\text {total, } 0-24 \mathrm{~h}}\right)$ to MIC ratios of $\geq 18,7$ and 4.5 were generated; with success defined as a regimen with a probability of target attainment of at least $80 \%$ at a given MIC.

Results: Thirteen studies $(\mathrm{n}=497)$ were eligible for study inclusion. The use of intermittent infusion tigecycline $150 \mathrm{mg}$ IV q12h for ward patients with resistant gram-negative bacteria up to a MIC of $0.48 \mu \mathrm{g} / \mathrm{mL}$ for an $\mathrm{AUC}_{\text {total, } 0-24 \mathrm{~h}} / \mathrm{MIC}$ target attainment of 18 , up to a MIC of $1 \mu \mathrm{g} / \mathrm{mL}$ for an $\mathrm{AUC}_{\text {total, } 0-24 \mathrm{~h}} / \mathrm{MIC}$ target attainment of 7 , and up to a MIC of $2 \mu \mathrm{g} / \mathrm{mL}$ for an $\mathrm{AUC}_{\text {total, } 0-24 \mathrm{~h}} / \mathrm{MIC}$ target attainment of 4.5 may be appropriate.

Conclusions: Resistant gram-negative bacteria infections that are associated with a tigecycline MIC $\geq 0.48 \mu \mathrm{g} / \mathrm{mL}$ may require treatment with alternate antibiotics, based on the failure to attain PK-PD tigecycline targets.

\section{Implementation and Suspension of an Antimicrobial Stewardship Audit and Feedback Program: Impact on Antimicrobial Utilization Patterns in an Inpatient General Internal Medicine Unit}

Wong $C^{1}$, Leung $E^{1,2}$

${ }^{1}$ University of Toronto, Leslie Dan Faculty of Pharmacy, Toronto, ON

${ }^{2}$ St. Michael's Hospital, Toronto, ON

Background: A goal of Antimicrobial Stewardship Programs (ASPs) is to optimize antimicrobial use; many ASPs use the audit and feedback (AAF) approach. Although AAF decreases unnecessary antimicrobial use, it is resource-intensive and may be difficult to sustain. There are limited data describing the long-term effects on antimicrobial use during suspensions in an ASP's AAF program.

Description: This retrospective study describes the implementation and subsequent temporary suspensions of AAF in the General Internal Medicine (GIM) unit at an urban teaching hospital. Data was collected over 65 months; during "active-AAF" time-frames, a dedicated, ID-trained clinical pharmacist and ID physician reviewed targeted antimicrobials from all GIM patients and made recommendations.

Action: GIM antimicrobial utilization was described by Defined Daily Doses (DDD) and costs (\$CAD), normalized per 1000 patient days. Data were compared two ways to assess immediate and long-term impacts of AAF activities (implementation and 2 temporarily suspensions): 1. All "active-AAF" time-frames were compared with "non-active AAF” (including pre-ASP), 2. Pre-ASP was compared with post-ASP).

Evaluation: Results are presented in Table 1. Comparing all "active-AAF" to all "non-active AAF" months, significant decreases $(\mathrm{P}<0.05)$ in DDD were observed for targeted antimicrobials: antipseudomonal antibacterials $(-29 \%)$ and fluoroquinolones (-56\%). A non-significant decrease (-6\%) was observed for overall antimicrobial DDDs. Comparing DDD utilization for during and Post ASP AF, post ASP led to an increase in the average utilization.

Implications: Our results show that temporary suspension of ASP AAF impacts antimicrobial utilization trends. Sustained decreases in targeted antimicrobials utilization were associated with active AAF. However, overall decreases in antimicrobial utilization were observed after initial implementation of ASP AAF (regardless of temporary suspension).

For the table that goes with this abstract, please see Abstract Appendix, available at https://www.cjhp-online.ca/index.php/cjhp/issue/view/188/ showToc 


\section{Assessment of a Therapeutic Drug Monitoring Strategy of Once Daily Dosing of Gentamicin/ Tobramycin in Paediatric Patients}

\author{
Chen $W^{1,2}$, Chung $E^{1,2}$, Strzelecki $M^{1,2}$, Boodhan $S^{1,2}$, Fecteau $A^{1}$, Seto $W^{1,2}$ \\ ${ }^{1}$ The Hospital for Sick Children, Toronto, ON \\ ${ }^{2}$ Leslie Dan Faculty of Pharmacy, University of Toronto, Toronto, ON
}

Background: Lack of evidence to support the use of once daily dosing (ODD) of aminoglycosides in paediatric patients. In July 2014, ODD was implemented for all eligible paediatric patients in a tertiary hospital. Objectives: To evaluate the ability of a once daily intravenous (IV) dose of $9 \mathrm{mg} / \mathrm{kg}$ gentamicin or tobramycin to achieve a target maximal concentration $\left(\mathrm{C}_{\max }\right)$ of $16-25 \mathrm{mg} / \mathrm{L}$, refine optimal sampling times, assess efficacy and safety in paediatric patients and compliance to the hospital's therapeutic drug monitoring (TDM) guideline.

Methods: Pharmacokinetic parameters were calculated from serum gentamicin or tobramycin levels drawn 3 and 6 hours after the aminoglycoside infusion and summarized using descriptive statistics. Monte-Carlo simulations based on calculated pharmacokinetic parameters were used to assess optimal dosing regimens.

Results: One hundred and forty children with 149 aminoglycoside courses were included. Mean pharmacokinetic parameters were: volume of distribution of $0.46 \pm 0.22 \mathrm{~L} / \mathrm{kg}$, clearance of $0.17 \pm 0.07 \mathrm{~L} / \mathrm{h} / \mathrm{kg}$ and half-life of $1.88 \pm 0.46 \mathrm{~h}$. Approximately half of the courses achieved $\mathrm{C}_{\max }$ target with the initial dose. Monte-Carlo simulations showed highest $\mathrm{C}_{\max }$ target attainment with $9 \mathrm{mg} / \mathrm{kg} /$ dose IV once daily. Approximately half of the empiric courses that did not reach $\mathrm{C}_{\max }$ target stepped down to oral antibiotics. Majority (77\%) of patients defervesced at the end of the course. No nephrotoxicity was identified. Almost all courses (99\%) used an initial dosing of $9 \mathrm{mg} / \mathrm{kg}$ as per formulary guideline. Approximately $66 \%$ of all courses screened had levels drawn only if patient received 3 or more doses of aminoglycosides. Only $34 \%$ of post-operative surgical patients had their levels drawn on or after post-operative day 2.

Conclusion: An initial aminoglycoside dose of $9 \mathrm{mg} / \mathrm{kg} /$ dose IV once daily is appropriate in achieving a $\mathrm{C}_{\max }$ of $16-25 \mathrm{mg} / \mathrm{L}$ and appears to be efficacious and safe in paediatric patients. Majority of courses complied with the TDM guideline.

\section{A Drug Use Evaluation of Aerosolized Ribavirin at a Canadian Teaching Hospital}

Attia $A^{1}$, So $M^{1,2}$, Wong $G^{1,2}$

${ }^{1}$ Leslie Dan Faculty of Pharmacy, Toronto, ON

${ }^{2}$ Toronto General Hospital, Toronto, ON

Background: Respiratory syncytial virus (RSV) causes life-threatening infections in immunocompromised hosts, leading to excess morbidity and mortality. Ribavirin is a guanosine analogue that is active against multiple viruses including RSV and is the cornerstone of treatment of RSV infections. Little is known about the patient characteristics that are most commonly treated with ribavirin at our institution.

Objective(s): The purpose of this investigation is to characterize the use of aerosolized ribavirin at our institution and report the clinical outcomes associated with its use.

Methods: Pharmacy records were accessed to identify the 61 patients for whom aerosolized ribavirin was dispensed from September $1^{\text {st }} 2016$ to September $1^{\text {st }} 2018$; 62 treatment courses were identified as one patient was treated twice. A retrospective chart review was conducted to obtain patient demographic and clinical data associated with aerosolized ribavirin treatment.
Results: The majority of patients who were treated with aerosolized ribavirin were immunosuppressed patients and lung transplant recipients. The primary indication for ribavirin was an RSV infection (98.4\%) and for those where follow-up polymerase chain reaction (PCR) results were available, $84.6 \%$ converted to a negative PCR result at 30 days.

Conclusion(s): Our results indicate that the most commonly treated patients were those who were immunosuppressed and/or lung transplant recipients. Aerosolized ribavirin was associated with a high 30-day PCR conversion rate (84.6\%), a low all-cause 30 -day mortality $(1.6 \%)$ and a low incidence of adverse effects $(6.5 \%)$, where dyspnea was the most common.

For the tables that go with this abstract, please see Abstract Appendix, available at https://www.cjhp-online.ca/index.php/cjhp/issue/view/188/ showToc

\section{Hypoglycemia in Paediatric Cardiology Patients Initiated on Propranolol: A Retrospective Review}

Matinnia $C^{1,2}$, De Castro $C^{1,2}$, Seto $W^{1,2^{*}}$

${ }^{1}$ Department of Pharmacy, The Hospital for Sick Children, Toronto, ON

${ }^{2}$ Leslie L. Dan Faculty of Pharmacy, University of Toronto, Toronto, ON

Underlined Author: Pharmacy Student working under Charisse De Castro and Winnie Seto

* Senior and Corresponding Author; sequence determines credit approach to authorship

Background: Propranolol is commonly prescribed in paediatric cardiology patients for arrhythmia, hypertension and TET spells. Paediatric patients, especially neonates, have an increased risk of hypoglycemia due to impaired and immature blood glucose regulation. Untreated hypoglycemia can lead to seizures, permanent brain injury, and potentially death. Propranolol has been shown to cause hypoglycemia but the extent and risk in paediatric patients with heart disease is unclear.

Objective(s): To describe the incidence of hypoglycemia and explore potential factors for development of hypoglycemia in paediatric and neonatal cardiology patients initiated on propranolol.

Methods: Retrospective chart review of cardiology patients aged less than 6 months, newly initiated on propranolol from September $14^{\text {th }}, 2016$ to September $23^{\text {rd }}, 2017$. Serum glucose, propranolol dosing, and risk factors for hypoglycemia (based on age-specific serum glucose ranges) were collected and analyzed by descriptive statistics. Univariate association between potential risk factors and hypoglycemia was assessed using chi-square analyses.

Results: Fifty patients on propranolol mostly for arrhythmias, TET spells, or hypertension with a mean starting dose of $0.87 \pm 0.27 \mathrm{mg} / \mathrm{kg} / \mathrm{dose}$ (range $0.13-1.14 \mathrm{mg} / \mathrm{kg} / \mathrm{dose}$ ). Five of 50 patients (10\%) developed hypoglycemia after propranolol initiation. Hypoglycemia onset was between $6 \mathrm{~h} 22 \mathrm{~min}$ and $38 \mathrm{~h} 53 \mathrm{~min}$ of propranolol initiation, where treatment ranged from none (self-resolved) to treated with dextrose/ sucrose/glucose. Most patients with baseline high serum glucose remained high or normal after propranolol initiation (89\%). One of 3 patients with baseline low serum glucose continued to display hypoglycemia after propranolol initiation. Eight patients were missing baseline serum glucose levels. There was no association between risk factors (sex, prematurity, age, indication, liver function, thyroid function) and hypoglycemia $(\alpha=0.05)$.

Conclusion(s): Hypoglycemia developed in $-10 \%$ of paediatric cardiology patients initiated on propranolol in current retrospective study, without significant association between risk factors and hypoglycemia. There is a need for standardized glucose monitoring and future study of a larger cohort for this population. 


\section{Anticholinergic Potential Risk Assessment Scales: Comparison of Drugs and Risk Scores}

\section{Chan $H^{1}$, Patel $T^{1,2}$}

${ }^{1}$ University of Waterloo School of Pharmacy, Kitchener, ON

${ }^{2}$ Centre for Family Medicine Family Health Team, Kitchener, ON

Background: Drugs with anticholinergic potential worsen cognition in older adults. Published anticholinergic drug scales help clinicians determine the severity of anticholinergic impact of certain drugs. However, the severity of anticholinergic potential for many drugs is rated inconsistently between the different scales. Furthermore, many of the drugs listed are often unavailable in Canada, decreasing the clinical utility of the scales.

Objectives: The goal of this project was to identify anticholinergic risk potential scales and compare the severity of anticholinergic potential rating between the different scales for the same drugs.

Methods: We conducted a literature search to identify scales that examine and assign an anticholinergic risk score to drugs. From each study, we extracted the methodology for developing the scale and their validation studies, if available, for comparison. We extracted and consolidated anticholinergic potential risk scores for all drugs, and stratified drugs based on the level of score concordance.

Results: We identified 9 scales in the literature search from which we retrieved 236 drugs with anticholinergic risk scores that are available in Canada. Most scales used a 4-point integer scale of 0-3 to rate anticholinergic risk, although there was significant variation in methodology used to assign the scores. The definition of "anticholinergic effect" was also different for each scale. Thirty-two (32) drugs were consistently identified as high anticholinergic risk across the scales, 18 as intermediate, and 18 as low risk. Anticholinergic risk of an additional 168 drugs could not be determined due to inconsistent scores or insufficient data.

Conclusions: Of the 236 drugs assessed for anticholinergic risk, the ratings were consistent for only 68 drugs between the different scales. Moving forward, we intend to establish a consensus on the anticholinergic severity of the 168 inconsistently rated drugs that are commonly used in Canada.

\section{Photosensitivity Associated with Long-Term Voriconazole Therapy: A Case Report}

\section{Cowley E, Reniers D}

London Health Sciences Centre, London, ON

Background: Adverse dermatologic reactions to voriconazole include fixed drug eruptions, cheilitis, discoid lupus erythematosus and Stevens-Johnson syndrome. In particular, less than $2 \%$ of patients develop photosensitivity reactions, presenting on sun-exposed areas of the body. Case Description: A 5-year old female diagnosed with acute lymphoblastic leukemia currently undergoing maintenance therapy developed a maculopapular erythematous rash on the scalp and face with excoriations. At the time, she was receiving trimethoprim-sulfamethoxazole (TMP/SMX), methotrexate, 6-mercaptopurine and voriconazole, all of which are known to be photosensitizing drugs. Voriconazole therapy was initiated for a suspected Aspergillus infection.

Assessment of Causality: Corticosteroids were initially prescribed for the affected areas, with minimal relief. A pediatric dermatologist suggested the rash was a photosensitivity reaction. The TMP/SMX was stopped and the rash persisted for several months thereafter. Voriconazole had been initiated approximately 12 months prior to the onset of the rash. The drug was stopped and the patient's caregiver reported that within 3 days the rash had cleared. The Naranjo scale was used to assess causality for the adverse reaction. A score of 7 identified voriconazole as a probable cause of the rash.

Literature Review: A case report previously identified 20 children who experienced photosensitivity reactions, with the majority having an underlying immunodeficiency. The onset of the reaction ranged from 2 weeks to 4.5 years on therapy. A retrospective analysis identified 5 patients with chronic graft-versus-host disease voriconazole-induced sensitivity mimicking a cutaneous flare.

Importance to Practitioners: Voriconazole-induced photosensitivity reactions are uncommon. Prompt recognition and drug discontinuation often facilitate improvement. Practitioners should screen for the potential concomitant use of photosensitizing drugs, and advise patients to avoid prolonged sun exposure, and the need for sunscreen.

\section{Safety of Enteral Nutrition Interruption around Levothyroxine Doses in Critically III Patients}

Ramandt $M^{1}$, Lougheed $C^{2}$, Kelly $L^{2}$, Pikul F', Miller $M^{2}$

${ }^{1}$ St. Joseph's Health Care, London, ON

${ }^{2}$ London Health Sciences Centre, London, ON

Background: Levothyroxine is recommended to be administered orally in a fasting state but few studies have examined the impact of enteral nutrition (EN) on absorption. However, ample evidence exists to demonstrate the importance of adequate feeding in critically ill patients. Practice at one hospital has historically varied between campuses, with nurses in one intensive care unit (ICU) generally holding EN two hours pre- and post- levothyroxine doses and nurses in the other ICU not interrupting EN.

Objective: Assessing our current state can help support the development of a consistent practice within the institution.

Methods: A retrospective chart review examined the safety implications of EN interruption around levothyroxine doses in critically ill patients. Eligible patients included adults admitted on levothyroxine to either ICU ( $\mathrm{N}=30$ per group). The primary outcome was the difference in the percentage of target daily EN volume received. Secondary outcomes included safety parameters (ventilator-free days, mortality, length of ICU stay and episodes of hypoglycemia potentially related to EN interruption). Descriptive statistics were calculated.

Results: Variability in practice exists both between and within sites. EN was held for a minimum of one hour pre- and post-dose for $87.3 \%$ of levothyroxine doses in one ICU compared to $0 \%$ of doses in the other $(\mathrm{p}<0.001)$. There was no EN rate adjustment in $20 \%$ of the cases where EN was held. The primary outcome of the average percentage of target daily EN intake received over the ICU stay was not significantly different between the two study groups ( $85 \%$ vs. $83 \%, p=0.804$ ). There were no statistically significant differences between the groups with respect to all secondary safety outcomes.

Conclusion: This study suggests that the inconsistent practices around the levothyroxine-EN interaction do not lead to differences in nutritional intake and a number of important safety outcomes. 


\section{Étude rétrospective des accidents et incidents associés à la documentation des doses de médicament dans un hôpital universitaire}

Videau $M^{1}$, Lebel $D^{1}$, Bussières J-F $F^{1,2}$

${ }^{1}$ Pharmacy Practice Research Unit, Pharmacy Department,

CHU Sainte-Justine, Montréal, QC

${ }^{2}$ Faculté de pharmacie, Université de Montréal, Montréal, QC

Contexte : Une erreur médicamenteuse peut se produire à n'importe quelle étape du circuit du médicament et conduire à un incident (I) ou un accident (A). Bien documenter les doses administrées grâce à la feuille d'administration des médicaments (FADM) dans le dossier patient est primordial

Objectif(s) : Décrire 14 ans d'I/A médicamenteux en lien avec la documentation des doses de médicaments.

Méthodologie : Il s'agit d'une étude descriptive rétrospective. Tous les rapports d'I/A médicamenteux extraits à partir de Gesrisk entre le $1^{\text {er }}$ avril 2004 au 31 mars 2018 ont été recueillis. Les I/A médicamenteux associés à la documentation des doses de médicaments ont été inclus lorsque les mots clés suivants ont été identifiés dans les données: «FADM», "profil», et la combinaison des mots «feuille» + «médicament». Un échantillon aléatoire de 50 rapports a été analysé de façon approfondie.

Résultats : Un total de 25705 rapports I/A médicamenteux ont été identifiés dont 25,7\% (6599) était associé à la documentation des doses. En 14 ans, cette proportion a doublé (16,2\% (263/1620) en 2004; $34,9 \%(524 / 1503)$ en 2018). Ces I/A liés à la FADM sont principalement dû à l'omission d'administration d'une dose $(2242 / 6599$, $34,0 \%)$, un mauvais horaire d'administration $(1148 / 6599,17,4 \%)$ et l'interception de l'erreur avant administration (947/6599, 14,4\%). Plus de la moitié des I/A liés à la documentation des doses sont de gravité C (4416/6599, 66,9\%). La mauvaise lecture de la FADM représente 49\% (23/47) des I/A audités.

Conclusion(s) : Vingt-six pourcent des I/A sont associés à la documentation des doses et cette proportion a augmenté au fil du temps. Cette hausse est possiblement associée à l'émergence d'outils électroniques qui permettent d'identifier plus facilement les divergences ainsi qu'à la mise en place de protocoles de soins de plus en plus complexes.

\section{Rapid Onset of Cholelithiasis in an Adult Treated with Ceftriaxone}

Abdelaziz H, Cormier N, Levesque T, St-Yves J, Al Habash M, Diaz O, Haberer $M$, Calugaroin D

Pharmacy Department, Campbellton Regional Hospital, Vitalité Health Network, Campbellton, NB

Background: Ceftriaxone is a third-generation cephalosporin with broad-spectrum antibacterial activity. Here, we report a case of ceftriaxone-induced cholelithiasis in an adult patient after a short period of administering ceftriaxone.

Case Description: A 57-year-old female was admitted to our hospital for meningitis and treated empirically with ceftriaxone $2 \mathrm{~g}$ every 12 hours. Other medications given included vancomycin, ampicillin and acyclovir. Based on culture results and a sensitivity report, ceftriaxone was continued while other medications were discontinued on day three after admission. Her liver function test (LFT) demonstrated an elevation in hepatic transaminases, and alanine and aspartate transaminases peaked on the fifth day (339 and 153 IU/L, respectively). Computed tomography (CT) and ultrasound (US) confirmed the presence of uncomplicated cholelithiasis. Ceftriaxone was discontinued and switched to cefotaxime $2 \mathrm{~g}$ every four hours. Hepatic transaminases started declining after ceftriaxone discontinuation and dropped to normal levels on day nine. After the administration of cefotaxime on the $25^{\text {th }}$ day, repeated US imaging revealed the persistence of biliary sludge. The patient was discharged in a good and stable clinical condition with follow-up planned at the outpatient clinic.

Assessment of Causality: When the concentration of ceftriaxone in the gallbladder exceeds the solubility of its calcium salt, precipitation occurs, forming a biliary sludge. Using the Naranjo Probability scale, the score was found to be 4, indicating a possible relationship between ceftriaxone and cholelithiasis.

Literature Review: Multiple case reports of ceftriaxone-induced cholelithiasis have been documented previously, most of which focused on children or on the prolonged use of ceftriaxone. However, our case report highlights the development of cholelithiasis in adults after administering ceftriaxone for a short time.

Importance to Practitioners: It is essential to monitor cholelithiasis and hepatic transaminase levels in both adult and pediatric patients receiving high doses of ceftriaxone.

\section{Evaluation of the Use of 'Do Not Use Abbreviations' in Hospital Orders: A Quality Assurance Audit}

Caron C, Arsenault P, Louis F

Ten Health Network, NB

Background: To determine the use of 'Do Not Use Abbreviations' (DNUA) in written medication orders in Horizon hospitals and compare results from 2017.

Description: Over a five-week period, audits were conducted across 12 hospitals on five different days of the week. All medication orders sent to pharmacy containing medication orders were reviewed and screened against the Institute for Safe Medication Practices (ISMP) list of Dangerous Abbreviations. Data collected from the 12 sites was analyzed and information about the audit results was then disseminated to staff.

Action: The audit was initially completed in August 2017 and repeated in 2018, to compare results and to detail improvement or lack thereof. Baseline results from 2017 were shared with prescribers, nursing and senior leadership. No further education was initiated.

Evaluation: In total, 15781 orders were received over the five-day audit period in 2018. Of these, 2360 (15\%) of orders contained an abbreviation on the list of DNUA. There was a $2 \%$ increase overall of the use of dangerous abbreviations. The most used DNUA were 'OD', 'D/C', and '@', with the most DNUA being seen in Fredericton, Moncton and Upper River Valley. The percentage of orders written by physicians remained the same over both years at $76 \%$, with nursing writing $16 \%$ of orders which decreased $1 \%$ from last year.

Implications: The use of DNUA in hospitals in New Brunswick seems to be increasing and therefore putting patients at increased risk of medication errors. A system to decrease the use of these abbreviations needs to be implemented and taken seriously by the involved parties to help ensure better patient safety in the province. 


\section{Leveraging the Electronic Health Record for Medication Safety Indicators}

Hollis K, Wong E, Gillert J, MacNeil M, Ellis J, Vaillancourt R The Children's Hospital of Eastern Ontario, Ottawa, ON

Background: In 2017, the institution implemented a fully electronic health record system. Medication safety indicators were chosen for both the Pharmacy and corporate level. These were primarily chosen to align with the Solutions for Patient Safety Adverse Drug Event Prevention roadmap.

Description: The Medication Safety Committee is comprised of representation from Pharmacy, Director of Nursing Practice, Medical Director of Patient Safety, Nursing Directors, Quality and Risk Management and Information Services. The committee selected the primary indicators to be monitored. The patient care leadership teams present on a regular basis their unit specific indicators and action plan.

Action: The hospital created practice standards for each indicator. In collaboration with the electronic health record reporting team, reports were developed to support monitoring and analysis of the indicators.

Evaluation: The Pharmacy medication safety indicators for September are: (1) medication cabinet restock scanning compliance - 96\%; (2) medication order validation turnaround time - 14 minutes for regular priority and 5 minutes for high priority orders; (3) dispense preparation barcode scanning compliance - 98\%; (4) medication reconciliation on admission within 48 hours $-85 \%$. The corporate indicators are: (5) smart pump drug library compliance -83 to $93 \%$; (6) barcode medication administration (BCMA) scanning compliance - 93\%; (7) allergy documentation prior to prescribing medications $-75 \%$; (8) medication incidents that reach the patient -21 events.

Implications: Consistent monitoring of medication safety indicators increases medication safety awareness and contributes to the achievement of reproducible positive results and the maintenance of highly reliable patient care.

\section{Implementation of Barcode Medication Administration Using a Quality Improvement Approach}

Vaillancourt R, Hollis K, Wong E, MacNeil M, Brooke R, Fantinic-Boyadjian $K, M a C$

The Children's Hospital of Eastern Ontario, Ottawa, ON

Background: In 2016, Barcode Medication Administration (BCMA) was added to the scope of our hospital's electronic health record (EHR) go-live planning. BCMA improves patient safety by decreasing medication administration errors.

Description: The goal was to reach $94 \%$ compliance for scanning both the medication and the patient.

Action: A team was formed jointly with Nursing and Pharmacy and a nurse was dedicated to the BCMA project for one year. A quality improvement approach was used to analyze and adjust workflows, EHR functions, devices, and physical space. Medication administration processes were identified using observation and group discussions. An FMEA was conducted for each proposed process. Using a human factors approach, we re-designed the unit medication rooms to fit additional workstations without compromising nursing workflow. We performed a 5 S concurrently. A $5 \mathrm{~S}$ is a method of organizing a work space for efficiency and effectiveness. Simulations of medication administration workflows were done with nurses and respiratory therapists to evaluate the mobile devices, which significantly influenced decision-making.
Evaluation: Initial BCMA rates, defined as scanning both the medication and patient, were $78 \%$. This increased as we made continuous improvements. The greatest improvement came several months when ownership of this safety initiative clearly transferred to clinical leadership of each unit. Scanning rates are now at $92.6 \%$ for scanning both patient and medication, 94\% for scanning medication only, and 95\% for scanning patient armband only. We also track scanning rates by unit, medication, and staff member. BCMA catches, on average, 70 near-miss safety events every day.

Implications: Scanning both medication and patient during barcode medication administration represents best practice in administration of medication in hospital. Our quality improvement approach involving extensive collaboration with nursing, FMEA, human factors analysis, and simulations of medication administration was key in the success of the project.

\section{Factors Influencing Prescribing of Direct Oral Anticoagulants in the Elderly Leading to Adverse Outcomes: An Analysis from the Windsor Region}

Vather $T^{1}$, Duronio $A^{1}$, Goh ${ }^{2}$, Yrigoyen-DaCruz $L^{1}$

${ }^{1}$ Department of Pharmacy, Windsor Regional Hospital, Windsor, $O N$

${ }^{2}$ Department of Statistics \& Actuarial Science, University of Waterloo,

Waterloo, ON

Background: Prescribing direct oral anticoagulants (DOACs) in elderly patients can be challenging due to their increased risk of renal insufficiency, polypharmacy and multiple comorbidities.

Objective: The primary objective of our study was to analyze patterns associated with prescribing of apixaban, rivaroxaban, and dabigatran in elderly patients admitted to our hospital with a bleeding-related adverse event.

Methods: A retrospective chart review was performed for patients 80 years or older admitted with a gastrointestinal, or intracranial hemorrhage (ICH) between April 1, 2015 and October 31, 2017. The best possible medication history confirmed patients were on a DOAC at admission. DOAC dose was assessed relative to product monograph recommendations. Descriptive and inferential statistics were used to analyze prescribing trends. A logistic regression analysis was performed to evaluate risk factors for being prescribed an inappropriate DOAC dose including gender, weight, creatinine clearance, HASBLED score, and concomitant antiplatelets, NSAIDs, or P-gp/3A4 interacting drugs.

Results: A total of 117 patients admitted with a gastrointestinal bleed or $\mathrm{ICH}$ were on a DOAC prior to admission. The majority of patients had atrial fibrillation and were prescribed rivaroxaban $(p<0.001)$. Forty-three percent of patients were prescribed an inappropriate DOAC dose, of which apixaban was significantly prescribed at lower doses $(p=0.02)$ and rivaroxaban at higher doses $(\mathrm{p}<0.001)$. Elderly patients with a creatinine clearance of $15-30 \mathrm{~mL} / \mathrm{min}$ were more likely to be prescribed inappropriate DOAC doses (OR: 5.57[95\% CI, 1.9-17.57]). Despite being prescribed the monograph-recommended dose, $57 \%$ of patients still experienced a bleeding event.

Conclusion: Elderly patients, especially those with severe renal impairment, are at risk of being prescribed an inappropriate DOAC dose. Most patients are under dosed on apixaban and overdosed on rivaroxaban. Our study suggests that the appropriateness of DOAC dose may not be the only factor contributing to adverse bleeding events. 


\section{Medication Reconciliation at Hospital Admission: Proactive versus Retroactive Models}

Levesque T, Abdelaziz H, Smith A, Cormier N, Bernard M, Lavoie S, Richard C, Dunn A, Gagnon J

Pharmacy Department, Campbellton Regional Hospital, Vitalité Health Network, Campbellton, NB

Background: The medication reconciliation (MedRec) process conducted at hospital admission uses either a proactive or retroactive model. Many hospitals have experienced challenges with MedRec, particularly its proactive model. Studies comparing the models are scarce.

Objective: The aim of this study was to analyze and quantify the retroactive and proactive MedRec models.

Methods: This prospective, observational study was conducted at Regional Hospital from May to June 2018. All of the patients undergoing MedRec during this time were included, except those who were discharged from the hospital, transferred to another hospital, or died within 48 hours of admission. The primary end point was to compare the time components of the process for the two models. The secondary end point was to determine the types of intentional and unintentional medication discrepancies identified and the frequency with which they occurred.

Results: After 249 MedRecs had been reviewed, 180 patients were enrolled. The total number of medications reconciled was 2118 . Of the 180 patients, 84 (46\%) were evaluated with the proactive model. There was no significant difference in the number of comorbidities identified $(p=0.282)$ or medications reconciled $(p=0.093)$ per patient for the two models. The median time from admission to the MedRec process was significantly shorter for the proactive model (48 minutes) than the retroactive model $(1135$ minutes $)(p<0.001)$. The percentage of documented intentional medication modifications in the proactive model $(16.3 \%)$ was more than twice that in the retroactive model $(7.3 \%)$ $(p<0.001)$. Patients evaluated by the proactive model had a significantly shorter hospital stay than those evaluated by the retroactive model $(p<0.001)$.

Conclusion: This study demonstrated that implementation of the proactive model was feasible. Compared with the retroactive model, the proactive model had a positive impact on preventing discrepancies with timeliness and efficiency.

\section{Assessing Medication Reconciliation in Hospitalized Adult Patients Discharged from Accountable Care Units in Saskatchewan Health Authority - Regina \\ Tri S, Mailman JF, Sweet K, Winkel T, Bell A, Semchuk B \\ Saskatchewan Health Authority, Regina Division, Regina, SK}

Background: Hospital discharge is a critical transition point for patients and may result in unintentional medication changes that increase potential or actual drug related problems, emergency department visits, readmissions, and preventable patient harm.

Objectives: To compare patients discharged from an Accountable Care Unit (ACU) using a standard versus non-standard discharge process on rates of overall unjustified medication discrepancies, Institute for Safe Medication Practices (ISMP) high risk medication discrepancies, involvement of the clinical pharmacist, and to identify potential causes of discrepancies.

Methods: Patients 18 years of age and older discharged during clinical pharmacist hours from one of two ACUs, one with (Unit A) and one without (Unit B) a standard discharge process, between October 10, 2017 and January 22, 2018.
Results: From our sample of 100 patients, 245 unjustified medication discrepancies were identified in 61 patients. Half of the patients from Unit A were discharged with at least one unjustified medication discrepancy versus $72 \%(36 / 50)$ from Unit B. The proportion ISMP high risk medication discrepancies were lower on Unit A than Unit B (7/49 vs. 35/196). Pharmacist documentation on Unit A was half that of Unit B (4/50 vs. $8 / 50$ ). The majority of unjustified medication discrepancies were omissions from Unit B (190/245). Unit A had a lower proportion of patients discharged with 5 or more unintentional medication discrepancies compared to Unit B (4/50 vs. 16/50).

Conclusions: More than half of patients were discharged from an ACU with at least one unjustified medication discrepancy. A standard discharge process decreased the proportion of unjustified discrepancies at discharge, including those involving ISMP high risk medications. Interpretation of clinical pharmacist impact was limited due a small proportion of documented pharmacist involvement. A standard process for medication reconciliation with improvement in documentation of rationale for medication may decrease the rate and impact of medication discrepancies.

\section{The Utilization of Splenectomy Post-Op Clinical Vaccinations Order Sets to Enhance Adherence and Timeliness of Vaccinations in Splenectomy Patients: A Pre-and-Post Intervention Study}

\section{Liao $X^{1}$, Fernandes $T^{1,2}$, Sengar $A^{2}$, Chiu $A^{1}$, Stockford $J^{1}$, Kapadia $N^{2}$ \\ ${ }^{1}$ Trillium Health Partners - Credit Valley Hospital, Mississanga, ON \\ ${ }^{2}$ Trillium Health Partners - Mississanga Hospital, Mississanga, ON}

Background: Asplenic patients are at increased risk of serious and life-threatening infections, especially by encapsulated pathogens. Over-whelming Post Splenectomy Infections (OPSI) are associated with an elevated mortality rate, however it can be easily prevented with appropriate vaccination prior to or post-splenectomy. Majority of asplenic patients $(60 \%+)$ have limited or no knowledge of their infection risk. The Splenectomy Post-Op Clinical Vaccinations Order Sets is one of the first initiatives to harmonized practice between sites at our organization and proved to be a success.

Objectives: This is a pre and post-intervention study aimed to assess the impact of the Splenectomy Post-Op Clinical Vaccinations Order Sets to improve practice adherence to recommended vaccination protocols outlined by the Canadian Immunization Guidelines among patients undergoing splenectomy.

Methods: A 5-year retrospective chart review was conducted in 2013 for the pre-intervention quantitative data. Another 5-year retrospective chart review was conducted in 2018 to determine post-intervention rates. The quantitative results were then tallied and analyzed.

Results: Prior to the implementation of the Splenectomy Post-Op Clinical Vaccinations Order Sets, $61 \%$ of patients at the organization received the required vaccinations. Post-intervention, vaccination rates increased to $93 \%$. Vaccinations were given on average, 10 days post-op, and most were given prior to hospital discharge. Qualitative analysis of order set showed excellent physician adoption rates within the hospital. Also, the initiative improved patient care by enhancing communication with primary care physicians via the structured discharge instructions included within the order set.

Conclusion: Vaccinations rates increased by $32 \%$ post-implementation of the Splenectomy Post-Op Clinical Vaccinations Order Sets, illustrating that a structured approach is necessary to improve clinical practice, enhance care and decrease potential life-threatening infections. 


\section{Utilization of Health Literacy Assessment Tools to Tailor Patient Counselling}

\author{
Dahri $K^{1,2,}$ Chan $S^{1}$, Spina $S^{3}$, McMinis $C^{4}, Z u k D^{4}$ \\ ${ }^{1}$ Faculty of Pharmaceutical Sciences, University of British Columbia, \\ Vancouver, $B C$ \\ ${ }^{2}$ Pharmacy Department, Vancouver General Hospital, Vancouver, BC \\ ${ }^{3}$ Vancouver Island Health Authority, Victoria, BC \\ ${ }^{4}$ Alberta Health Services, Calgary, $A B$
}

Background: Patients with low health literacy experience difficulty in understanding their medications leading to overall poorer health outcomes. Pharmacists can utilize different strategies to better tailor their patient education for these individuals. However, in order to utilize these strategies, they need to be able to effectively identify these patients. Without the use of formal assessment tools, health care practitioner's identification of patients with low health literacy is poor. It is unclear whether pharmacists currently utilize health literacy screening tools or if they incorporate different counselling strategies in this patient population.

Objectives: To characterize pharmacists understanding of health literacy and their use of screening and counselling strategies before and after completion of an educational module. To identify barriers that pharmacists perceive to exist that prevents them from using health literacy tools.

Methods: Pharmacists in three health authorities were administered a pre-survey and then given access to an online 10-minute educational video. The post-survey was sent 1 -month later. Descriptive statistics were used to quantify survey responses with comparisons made between pre and post responses.

Results: There were 131 respondents for the pre-survey and 39 for the post-survey. In the pre-module survey, $83 \%$ of pharmacists felt they understood what health literacy was but only $52 \%$ currently assessed patients for their health literacy status and $40 \%$ were aware of what strategies to use in low health literacy patients. Lack of time (74\%) was the biggest barrier in assessing patients' health literacy. In the post-module survey, $87 \%$ felt they understood what health literacy was and $64 \%$ incorporated health literacy status evaluation into their clinical practice. The educational module was helpful to the clinical practice of $74 \%$ of respondents.

Conclusion: A short educational intervention can improve pharmacists' understanding of health literacy. Time remains the biggest barrier in improving care of these patients.

\section{Development of an Inpatient Pharmacist Diabetes Educator Role}

Halapy $H^{1,2}$

${ }^{1}$ St. Michael's Hospital, Toronto, ON

${ }^{2}$ Leslie Dan Faculty of Pharmacy, University of Toronto, Toronto, ON

Background: Consistent with Canadian Diabetes Association Clinical Practice Guidelines' recommendations, inpatient diabetes education is essential for patients newly diagnosed with diabetes to self-manage insulin injections in the community.

Description: A pharmacist inpatient diabetes educator role was developed, implemented, and reviewed.

Action: A previous evaluation indicated a need for patient- and systemslevel approaches to inpatient diabetes education (e.g. patient consultations, staff education, systems enhancements). A pharmacist was considered ideal to fulfill this new role due to his diabetes drug knowledge. A pharmacist-led inpatient diabetes consultation service was implemented to provide diabetes education (e.g. new diagnosis, insulin starts/changes, meter teaching, diabetic ketoacidosis management), assist in blood glucose management, and help facilitate discharge. Staff education was provided (e.g. hypoglycemia management, insulin pen usage, insulin formulary recommendations) along with systems enhancements (e.g. insulin pen rollout, hypoglycemia protocol, insulin order set revisions).

Evaluation: Review of the role after 9 months indicated the following: 142 patient consultations were completed; consults averaged 4 per week (range 1 to 9); average consult time 90 to 120 minutes (range 30 to 240 minutes); most consults required 1 visit to complete (range 1 to 5 visits); $49 \%$ of consults were for new insulin starts followed by complex diabetes management (23\%), glucometer teaching (11\%), diabetic ketoacidosis management recommendations (7\%), insulin pump management (4\%); $56 \%$ of consultations were seen the same day. Only 8 patients seen for consultation were readmitted for diabetes related issues (e.g. insulin non-adherence). Regarding systems-level approaches, the diabetes pharmacist led: 11 inpatient staff education sessions; conversion of the institution to insulin pen administration; implementation of 5 new insulin order sets; and removal of all insulin sliding scales from institutional prescribing system.

Implications: The new inpatient diabetes pharmacist role added to the institution's quality dimensions by providing timely diabetes education, provided staff education, and implemented several key diabetes initiatives.

\section{Analyse descriptive des publications dans le domaine de la pharmacie de 1973 à 2016}

Chabrier $A^{1}$, Lariviere $V^{2}$, Lebel $D^{1}$, Bussières J-F

${ }^{1}$ CHU Sainte-Justine, Montréal, QC

${ }^{2}$ Université de Montréal, Montréal, QC

Contexte : La publication scientifique permet de commenter l'évolution d'une discipline.

Objectif(s) : Décrire et commenter la publication scientifique dans le domaine de la pharmacie et son évolution dans le temps.

Méthodologie : Étude descriptive rétrospective. Extraction réalisée à partir de l'index de citations Web of Science (WOS) à partir des critères: discipline (Clinical medicine) et spécialité (pharmacy). Analyses réalisées à l'aide d'un chiffrier (Excel ${ }^{\mathrm{MD}}$ ) en fonction de l'année publication, de la revue, du pays de l'auteur correspondant, du pays et de la langue de la revue.

Résultats : Un total de 103538 articles ont été publiés, dans 40 revues, de 1973 à 2016 dans la spécialité "pharmacy ", représentant 1,09\% de la discipline " clinical medicine ». Cette proportion diminue dans le temps ( $2.43 \%$ en 1973 vs $0.72 \%$ en 2016 ). Le top- 5 des auteurs correspondants depuis $1977(\mathrm{n}=80145)$ proviennent du Japon (23\%), États-Unis d'Amérique (21\%), Allemagne (9\%), Chine (6\%) et Italie (5\%). Le Canada est au $15^{\text {eme }}$ rang (1\%). Le top-5 des journaux ayant le plus de publications sont: Chem. Pharm. Bull (20\%), Int. J. Pharm (15\%), Pharmazie (11\%), Eur. J. Med. Chem. (9\%) et Yakugaku Zasshi (7\%). Le seul journal canadien représenté est le Can J Pharm Sc ( $\mathrm{n}=280$ articles, $<1 \%)$. Parmi les revues utilisées par les pharmaciens hospitaliers, on retrouve Am J HealthSys Pharm (6,9\%), Drug Saf (2\%), DICP (2\%), PWS (1\%). Les publications sont principalement en anglais (93\%), en allemand $(6 \%)$ et dans d'autres langues $(1 \%)$. Certaines revues populaires en pharmacie ne sont pas recensées dans l'étude car elles ne comportent pas le terme "pharmacy » dans leurs mots-clés.

Conclusion(s) : La proportion des articles codés "Pharmacy» est en diminution depuis 1973. Le Canada représente une part marginale de ces publications dans WOS. 


\section{Face and Content Validation of an Instrument to Measure Medication Management Capacity in Older Adults}

\author{
McDougall $A^{1}$, Ivo $\mathcal{F}^{2}$, Kooner $A^{3}$, Bauer $J^{4}$, Pritchard $S^{4}$, Chang $F^{2}$, \\ Patel $T^{2,4}$ \\ ${ }^{1}$ University of Waterloo Faculty of Applied Health Sciences, Waterloo, ON \\ ${ }^{2}$ University of Waterloo School of Pharmacy, Kitchener, ON \\ ${ }^{3}$ University of Waterloo Faculty of Science, Waterloo, ON \\ ${ }^{4}$ Centre for Family Medicine Family Health Team, Kitchener, ON
}

Background: Numerous medication adherence products are marketed to improve medication adherence. However, no tools currently address the evaluation of domain-specific limitations among older adults managing medications.

Objectives: The objective was to investigate face and content validation of the Domain Specific Limitation in Medication Management Capacity Instrument, a tool developed to measure domain-related problems among older adults self-managing medications. Domains and subdomains in this instrument were 1. physical abilities (vision, dexterity, hearing); 2. cognition (comprehension, memory, executive functioning); 3. medication regimen complexity (dosing regimen, non-oral administration, polypharmacy); and 4. access/caregiver (prescription refill, new prescription, caregiver).

Methods: Healthcare professionals, recruited through purposive sampling, assessed each variable within domain and sub-domains for relevance, importance, readability, understandability, and representation. Percent agreement and content validity index (CVI) for each variable was determined to examine face and content validity.

Results: Twelve pharmacists participated in the study, of whom $83 \%$ were female with a mean of 12.4 years of practice. Domain-specific percent agreement for relevance were Physical Abilities 98\%, Cognition $100 \%$, Medication Regimen Complexity 90\%, and Access/Caregiver 92\%. Domain-specific percent agreement for importance were Physical Abilities 98\%, Cognition 100\%, Medication Regimen Complexity 88\%, and Access/Caregiver 90\%. All domain relevance and importance CVI scores were $100 \%$. Sub-domain percent agreement scores were above $80 \%$ except for relevance of hearing $(73 \%)$, and importance for hearing (66\%), and new prescription (75\%). Similarly, sub-domain CVI scores were above $80 \%$ except for importance of new prescription $(73 \%)$. Eighty-three percent (35/42) of variables maintained relevance and $79 \%$ (33/42) maintained importance CVI scores above $80 \%$. Fifteen variables were either removed (8) or modified (7) due to low CVI scores for at least one measurement (relevance, importance, readability or understandability).

Conclusions: This tool can be used to assess and identify domain and sub-domain specific limitations in medication management capacity among older adults.

\section{Improving Pharmacist-Pharmacist Communication at Hospital Discharge}

\author{
Varghese $S^{1,2}$, Guilcher $S^{1}$, Luke $M^{2}$, Visentin $\mathcal{F}^{2}$, Fernandes $O^{1,3}$, \\ McCarthy $L^{1,2}$ \\ ${ }^{1}$ University of Toronto, Toronto, $\mathrm{ON}$ \\ ${ }^{2}$ Women's College Hospital, Toronto, ON \\ ${ }^{3}$ University Health Network, Toronto, ON
}

Background: Patients are particularly vulnerable during transitions in care, often due to miscommunication between health care providers. PROMPT, an intervention focused on improving communication between hospital and community pharmacists, facilitates medication management at the time of hospital discharge.
Objective: To assess the fidelity, the degree to which the intervention was delivered as intended, and community pharmacist perceptions of PROMPT.

Methods: Descriptive feasibility study (Jan - Apr 2018). Setting: General Internal Medicine units. Patient eligibility criteria: adults $\geq 18$ yrs, admitted to a general internal medicine unit for $\geq 72$ hours, taking $\geq 5$ medications when discharged home. Intervention: Hospital pharmacists fax a patient's usual community pharmacy their discharge prescriptions and medical discharge summary (when available) with their contact information and follow-up by telephone. Data Collection: Process tracking forms (fidelity), Phone surveys (community pharmacists' perceptions about the intervention).

Results: Forty-five patients received the intervention provided by 12 hospital pharmacists across two hospitals. Forty-two phone surveys were completed with 37 unique pharmacies. Intervention fidelity was suboptimal: $78 \%(35 / 45)$ of interventions did not include discharge summaries, $24 \%(11 / 45)$ of discharge packages did not include contact information, and 3 process tracking forms were excluded because the discharge prescriptions were provided to patients directly. On a 9-point Likert-type scale, community pharmacists believed all components of the intervention were extremely important, including the contact information (median 9, IQR: 0), the discharge summary (median: 9, IQR: 1), and the follow-up phone call (median: 9, IQR: 2).

Conclusion: The high ratings of the importance of PROMPT suggest that community pharmacists view the program favorably. To improve fidelity, recommendations include broadening eligibility criteria to increase enrollment and providing iterative feedback to hospital pharmacists during data collection. Areas for future research include interviews with hospital and community pharmacists to gain in-depth feedback about PROMPT and assessing fidelity at additional sites.

\section{Exploring the Perspectives of Healthcare Professionals in Delivering Optimal Oncology Medication Education}

Lively $A^{1}$, Minard $L^{1}$, Scott $S^{1}$, Deal $H^{2}$, Lambourne $T^{3}$, Giffin ${ }^{4}$ ${ }^{1}$ Department of Pharmacy, Nova Scotia Health Authority (Central Zone), QEII Health Sciences Centre, Halifax, NS

${ }^{2}$ College of Pharmacy, Dalhousie University, Halifax, NS

${ }^{3}$ Department of Pharmacy, Nova Scotia Health Authority (Northern Zone), Colchester East Hants Health Centre, Truro, NS

${ }^{4}$ Nova Scotia Health Authority (Central Zone), QEII Health Sciences Centre, Halifax, NS

Background: Patient education is an important component of chemotherapy treatment as it has been shown to positively impact patients who receive it. This education is often provided by multidisciplinary teams with the goal of improving patient care. However, informational discrepancies exist in what healthcare professionals prioritize compared to the patients they treat. Generally, each healthcare professional has their own approach and chooses the amount of information provided to patients. To optimize patient education, it is important to understand what healthcare professionals perceive to be ideal oncology medication education for patients to receive and what they feel is their role and the role of others in its delivery. Few studies have explored the roles of healthcare professionals in delivering oncology medication education and it is unknown what healthcare professionals at Nova Scotia Health Authority - Central Zone (NSHA) perceive as their role in this.

Objective: To explore the perspectives of healthcare professionals working in medical, gynaecological or hematological oncology to identify what they perceive to be optimal oncology medication education for patients. 
Methods: Healthcare professionals (physicians, nurses and pharmacists) working in medical, hematological or gynaecological oncology at NSHA were invited to participate in one-on-one semi-structured interviews which were audio-recorded, transcribed and analyzed using thematic analysis.

Results: Fifteen interviews, including five physicians, four nurses and six pharmacists were conducted from February to April 2018. Four major themes were identified; Delivery of oncology medication education, Facilitating the patient learning process, Multidisciplinary approach and Understanding barriers to the healthcare professional in providing education.

Conclusion: Identified themes uncovered some previously unknown ideas about how healthcare professionals felt education could ideally be delivered to patients and also supported some of the findings in the literature. The data obtained will inform the design of any new models for oncology medication education at this site and potentially others.

\section{Development and Implementation of a Competency Assessment Tool for Hospital Pharmacists}

Gallagher B, Dumont Z, Semchuk B

Saskatchewan Health Authority, Regina, SK

Background: One of pharmacists' greatest contributions to patient care is pharmacotherapeutic knowledge and skills, regardless of setting. The "Oath of a Pharmacist" states, "I will accept the lifelong obligation to improve my professional knowledge and competence". At present, no Canadian hospital pharmacy specific competency assessments are published. We set out to develop a tool in our institution to ensure ongoing competency assessment and aid in staff development.

Description: Departmental competency assessment during training evolved over a decade to become an activity based checklist, which included a standardized tool for objectively quantifying order processing. While useful, it was not comprehensive in assessing all aspects of pharmacy practice.

Action: Literature review focused on ongoing assessment of pharmacist competency. Further, we sought input from Canadian hospital colleagues. In conjunction with the American Society of Health System Pharmacist's competency textbook and the Canadian Pharmacy Residency Board standards, our existing checklist was redeveloped. Metrics were established in alignment with local practice to aid in assessment and tracking growth.

Evaluation: A scarcity of literature in ongoing assessment of pharmacist competency was noted. Our new tool has been used for trainee pharmacists and existing staff during work-withs. It has received positive feedback from trainers and trainees. In addition to tracking metrics, all sections of the tool include room for comments. If deficiencies are noted, a detailed action plan based on assessment results is to be developed.

Implications: Our tool is objective and better allows us to determine if individuals are meeting and maintaining competencies. Additionally, it serves to orientate staff to the department and helps them to feel prepared to practice in our facility. Though not validated, the tool is based on existing literature and may serve as a framework for others to adapt within their sites. Future work will focus further on clinical competencies.

\section{Transition to Insulin Pens in Inpatient Rehabilitation and Mental Health Care Hospitals}

Neilans $L^{1}$, Van Berkum $A^{1}$, Countryman $T^{1}$, Cummings $M^{1}$, Blokker $M^{1}$, Ouellette $V^{2}$

${ }^{1}$ St.Joseph's Health Care London, London, ON

${ }^{2}$ University of Waterloo, Waterloo, $O N$

Background: Insulin is a high-alert medication that can cause significant harm when used in error. The"2017 Institute of Safe Medication Practices(ISMP) Guidelines for Optimizing Safe Subcutaneous Insulin Use in Adults", highlight best practices, including the recommendation to dispense insulin as patient-specific. Insulin pens have several advantages over insulin vials: more accurate dosing, less needle stick injury, less waste of insulin, less painful injection and they enable patient teaching. Insulin pens carry a risk of disease transmission if shared between patients, enhancing the need for education and safe dispensing practices.

Description: A joint collaboration between Pharmacy and Professional Practice was initiated to improve insulin safety. We implemented the transition from ward-stock insulin vials to patient-specific insulin pens across three of our inpatient sites (Physical Rehabilitation, Mental Health Care and Forensic Mental Health Care).

Action: We audited our insulin dispensing practices and compared these to best practice. We met and surveyed other hospitals, performed a cost analysis, developed dispensing workflows and engaged key stakeholders. All insulin vials and ward-stock insulin were removed and insulin is now dispensed as patient-specific insulin pens. Nursing staff completed a learning module on insulin pen safety and administration, followed by a return demonstration.

Evaluation: At 2 months post-transition, $80 \%$ of nursing staff have completed the mandatory education. Insulin scanning rates from 2 months prior compared to 2 months post-transition have either remained the same or increased ( Physical Rehabilitation: $88 \%$ prior, $89 \%$ post; Mental Health Care: $45 \%$ prior, $43 \%$ post; Forensic Mental Health Care: $79 \%$ prior, $93 \%$ post). Insulin medication errors are being tracked before and after the transition.

Implications: Several novel insulin products are only marketed as a pre-filled pen, compelling inpatient facilities to develop safe dispensing of these products. The transition to patient-specific insulin pens aligns with ISMP recommendations, provides consistent dispensing and enhances patient safety.

\section{Point Prevalence Survey of Benzodiazepine and Sedative-Hypnotic Drug Use in Long-Term Care}

Gehrig $M^{1}$, Canning $T^{2}$, Lambourne $T^{1}$, Neville $H^{1}$, Bowles $S^{1,2}$

${ }^{1}$ Nova Scotia Health Authority, NS

${ }^{2}$ College of Pharmacy, Dalhousie University, Halifax, NS

Introduction: Benzodiazepines and sedative-hypnotic drugs (BZD/ SHD) are associated with cognitive impairment and falls among frail, older adults. While the use of these agents is well-known in communitydwelling older persons, prevalence of BZD/SHD use in long-term care residents is less understood although hypothesized to be high.

Objectives: To identify the point prevalence of BZD/SHD use in a 146 bed long-term care (LTC) facility and to explore the relationship between a fall history and current BZD/SHD use.

Methods: A point prevalence survey (PPS) was conducted by reviewing the health records of adults in a LTC facility. BZD/SHD utilization was defined as having received an oral BZD/SHD within the 24 hours prior 
to the survey. Patient demographics, BZD/SHD utilization, indication, use prior to LTC admission and fall history in the past 6 months were collected. Results were summarized descriptively.

Results: Overall prevalence of BZD/SHD use was 45.2\% (66/146). The average age of residents was 91 years old, $87.9 \%$ of BZD/SHD users were $\geq 85$ years old and $28.8 \%$ were $\geq 95$ years old. The most commonly used drugs for residents receiving at least one $\mathrm{BZD} / \mathrm{SHD}$ were trazodone $(39.5 \%)$ and melatonin (38.3\%). The majority of use $(71.6 \%)$ had been initiated after admission to the LTC facility. In the past 6 months, $44.3 \%$ (47/106) of residents had at least one fall and 53.2\% (25/47) of those residents were currently taking a BZD/SHD.

Conclusions: BZD/SHDs were used by $45 \%$ residents in the LTC facility. Areas identified for quality improvement included investigating strategies to minimize BZD/SHD use in the LTC setting, particularly for those with a history of falls; and ensuring that when BZD/SHD use is appropriate, it is used at the lowest effective dose and for the shortest duration of time.

\section{Benzodiazepine and Sedative-Hypnotic Drug Use in Hospital: Perspectives from Healthcare Providers \\ Neville $H^{1}$, Granter $C^{2}$, Adibi $P^{2}$, Belliveau $J^{1}$, Isenor ${ }^{2}$, Gehrig $M^{1}$, Bowles $S^{1,2}$ \\ ${ }^{1}$ Nova Scotia Health Authority, NS \\ ${ }^{2}$ College of Pharmacy, Dalhousie University, Halifax, NS}

Background: A point prevalence survey (PPS) conducted in the province's acute care hospitals found that the overall prevalence of benzodiazepine and sedative-hypnotic drug (BZD/SHD) use was 35\%, with almost $40 \%$ of these drugs being newly started in hospital. A scoping review of interventions to decrease $\mathrm{BZD} / \mathrm{SHD}$ use in hospitals is needed to determine current evidence and research gaps.

Objective: To gauge the opinions of healthcare providers and patients on BZD/SHD used for sleep and anxiety in hospital to inform a scoping review.

Methods: Healthcare providers, patients and their families were invited, via a standardized email, to participate in stakeholder meetings being held in person at the hospital from May 1 to May 4, 2018. Stakeholders were asked open-ended questions surrounding their experience with sleeping pill use in the hospital, how to change sleeping pill use, other options for insomnia or anxiety, and resources available to decrease $\mathrm{BZD} / \mathrm{SHD}$ use in the hospital. Meetings were recorded, transcribed, and coded into categories, sub-categories and keywords. Results were summarized descriptively.

Results: Twenty-one participants attended three group meetings and two individual meetings. Nurses, physicians and pharmacists most commonly discussed solutions and barriers. Top solutions were related to the need for education, finding alternatives to BZD/SHD use, medication reviews, discussing tapered withdrawal with the patient, and improving the hospital environment to promote sleep. Barriers identified included a disruptive hospital environment, the ease of prescribing BZD/SHDs, difficulty making changes to home medications and staff not being aware of the risks of using BZD/SHDs.

Conclusions: A number of barriers and solutions for improving BZD/SHD drug use in hospital were identified. Stakeholder feedback will be used to inform both the scoping review and potential interventions that can be implemented to reduce BZD/SHD in hospital.

\section{Investigation of the Average Duration of Dual Antiplatelet Therapy in Dialysis and Pre-Dialysis Patients}

Dhawan A, Liao R

Trillium Health Partners - Credit Valley Hospital, Mississanga, ON

Background: The use of dual antiplatelet therapy (DAPT) with aspirin and a $\mathrm{P} 2 \mathrm{Y}_{12}$ receptor antagonist has been well-studied in the secondary prevention of acute coronary syndromes (ACS). Both the American and Canadian DAPT guidelines broadly recommend a duration of 12 months of DAPT post-ACS, with recent literature recommending up to 3 years of DAPT in certain clinical scenarios. Although chronic kidney disease $(\mathrm{CKD})$ is a known risk factor for both thrombotic and bleeding events, studies evaluating the optimal duration of DAPT often exclude or minimally represent CKD patients.

Objectives: This study examined the average duration of DAPT, clinical factors impacting DAPT duration and whether DAPT was being reassessed in hemodialysis, peritoneal dialysis and pre-dialysis patients at a hospital with a regional dialysis centre.

Methods: A retrospective chart review was performed in adult CKD patients that suffered an ACS event and started DAPT between January 1, 2014 and March 1, 2017.

Results: The average duration of DAPT was 366 days $(\mathrm{N}=65)$, with a bimodal distribution, defining two subgroups - those on DAPT for $<365$ days (33 patients) and those on DAPT for $>365$ days (32 patients). Documentation of a bleed/increased bleed risk was the primary reason DAPT was stopped before 1 year. Statistically significant factors associated with a shorter DAPT duration ( $<365$ days) included concomitant use of medications that increase bleeding risk. Both proton pump inhibitor use and having peripheral artery disease were statistically significant factors associated with a longer DAPT duration ( $>365$ days). Reassessment of DAPT by the renal pharmacist was documented for $28 \%$ of patients.

Conclusion: DAPT duration in CKD patients is highly variable and impacted by numerous factors. These results point toward the need for further investigation of optimal DAPT duration and a more tailored reassessment of DAPT in the $\mathrm{CKD}$ population.

\section{Revue de l'utilisation de l'eculizumab}

Franck $B^{1}$, Beauchemin $M^{2}$, Bédard $P^{2}$, Teira $P^{3}$, Lapeyraque $A L^{4}$, Bussières J-F $F^{1,2}$

${ }^{1}$ Unité de recherche en pratique pharmaceutique, CHU Sainte Justine, Montréal, QC

${ }^{2}$ Département de Pharmacie, CHU Sainte Justine, Montréal, QC ${ }^{3}$ Service d'hémato-oncologie, CHU Sainte Justine, Montréal, QC ${ }^{4}$ Service de néphrologie, CHU Sainte Justine, Montréal, QC

Contexte: L'éculizumab est indiqué dans le traitement des hémoglobinuries paroxystiques nocturnes (HPN) et des syndromes hémolytiques urémiques (SHU) atypiques.

Objectif(s): Décrire l'utilisation de l'éculizumab dans un centre hospitalier universitaire.

Méthodologie: Étude descriptive rétrospective des patients ayant reçu de l'éculizumab du 1-1-2011 au 1-6-2018. Des données liées aux caractéristiques démographiques (genre, poids, âge), au diagnostic (étiologie, critères diagnostic, durée d'hospitalisation), aux conditions de prescription et d'administration (vaccination, antibioprophylaxie, dose, durée et coût du traitement) et à l'évolution (réhospitalisation, retraitement, survie) ont été recueillies. 
Résultats: 29 patients (20 filles, 9 garçons) ont reçu au moins une dose d'éculizumab soit 22 patients avec microangiopathie thrombotique (MAT), (16 MAT secondaires, quatre SHU secondaires à une infection à E.coli producteur de Shigatoxine, deux SHU atypiques), une HPN, cinq glomérulonéphrites à $\mathrm{C} 3$, un rejet humoral de greffe de rein. Lâge médian au traitement était de 8 ans [ $\min =1-\max =39]$. 21 patients $(72 \%)$ étaient hospitalisés au diagnostic avec une durée médiane d'hospitalisation de 39jours [1-464]; 16 patients (59\%) ont dû être hospitalisés en soins intensifs pour une durée médiane de 13jours [1-59]. Dans les cas de MAT secondaire, dix traitements $(62,5 \%)$ suivent une greffe de moelle, deux $(12,5 \%)$ une chimiothérapie sans greffe, un $(6,25 \%)$ une hypertension artérielle, deux (12,5\%) sont d'origine indéterminée et huit patients présentaient une réaction du greffon contre l'hôte associée. 100\% des patients ont été vaccinés contre le méningocoque ou ont reçu une antibioprophylaxie. Les patients ont reçu un nombre médian de doses de 6 [1-172] sur une durée médiane de 93 jours [1-2778]. Sept patients $(24,1 \%)$ ont dû être hémodialysés et 14 (48,3\%) réhospitalisés. Un coût médian/patient de 114884 [7180\$-3755260\$] a été calculé.

Conclusion(s): L'éculizumab a été utilisé dans trois cas selon les indications du fabricant et dans 26 cas hors-monographie. Il est nécessaire d'utiliser un programme d'encadrement de l'utilisation émergente

\section{Preliminary Evaluation of the NAPRA 6-hour Rule for Single-Use Vials after First Puncture in an ISO-5 Environment}

Perks $W^{1}$, Carating $H^{1}$, Charbonneau $L F^{1}$, Mascioli $M^{1}$, Iazzetta $J^{1}$, Stinson $J^{1}$, Nedzka-Rajwans $I^{1}$, Walker $S E^{2}$

${ }^{1}$ Department of Pharmacy Sunnybrook Health Sciences Centre, Toronto, ON ${ }^{2}$ Leslie Dan Faculty of Pharmacy, University of Toronto, Toronto, ON

Background: The USP-797 and NAPRA guidelines related to the 6-hour Beyond-Use-Dating (BUD) limit is not aligned with other BUD guidelines (11-days) for a low-risk product compounded from a single use vial.

Objective: To evaluate sterility of single use vials test following multiple withdrawals over a 7 day sampling period.

Methods: One 20-mL vial containing sterile TSB growth medium was placed in each of 5 laminar air flow hoods weekly. 1-mL samples were drawn from each vial immediately after placement in the hood and at 48 and 168hours. Prior to sample withdrawal vials were visually inspected for turbidity. After 1 week, vials were collected, incubated at $37 \mathrm{oC}$ for 14-days and inspected visually every 2 days for evidence of contamination (turbidity). For positive controls, three TSB vials were inoculated with less than 102 of S.epidermidis ATC12228. As a negative control, three unopened vials of TSB were incubated for 14 days. The contamination rate was calculated based on the $95 \%$ confidence interval constructed around the observed contamination rate per 100 vials and per 100 transfers.

Results: All positive control vials demonstrated growth within 48-hours. All negative control vials showed no growth. During the first 20 -weeks of monitoring, all vials (100 vials - 300 transfers) remained sterile following storage at room temperature for 7 days and subsequent incubation for 14 -days. The $95 \%$-CI of the contamination rate is 0.000 to $0.017 \%$ per transfer.

Conclusions: Single-use-vials, aseptically punctured within an ISO-5 environment, maintain sterility following multiple withdrawals over 7-days.

\section{Physical Compatibility and Stability of Ascorbic Acid Injection in Polyvinyl Chloride Minibags at $4^{\circ} \mathrm{C}$ and Room Temperature $\left(25^{\circ} \mathrm{C}\right)$}

Iazzetta $J^{1}$, Law $S^{1}$, Adhikari $N^{3}$, Lamontagne $F^{4}$, Kanji $S^{2}$, Walker $S E^{5}$ ${ }^{1}$ Department of Pharmacy, Sunnybrook Health Sciences Centre, Toronto, ON

${ }^{2}$ The Ottawa Hospital, Departments of Pharmacy and Critical Care Medicine, Ottawa, $O N$

${ }^{3}$ Department of Critical Care Medicine, Sunnybrook Health Sciences Centre, Toronto, $O N$

${ }^{4}$ Department of Medicine, Université de Sherbrooke, Sherbrooke, QC ${ }^{5}$ Leslie Dan Faculty of Pharmacy, University of Toronto, Toronto, ON

Background: Emerging data suggests intravenous ascorbic acid (AA) may be a potential therapy in patients presenting with severe sepsis. Stability of diluted AA is not known beyond 24 hours.

Objective: To evaluate the physical compatibility and chemical stability of AA injection diluted in either $50 \mathrm{~mL}$ NS or D5W PVC minibags during storage over 14 days at $25^{\circ} \mathrm{C}$ and at $4^{\circ} \mathrm{C}$, protected from light (PFL).

Methods: On study day $0,36 \mathrm{mg} / \mathrm{mL}$ and $77 \mathrm{mg} / \mathrm{mL}$ concentrations (Sandoz) and $40 \mathrm{mg} / \mathrm{mL}$ and $92 \mathrm{mg} / \mathrm{mL}$ concentrations (Mylan) of AA were prepared and PFL. 3 units of each container were stored at room temperature and 3 were stored in the refrigerator. Concentration and physical inspection were completed on study days $0,0.33,1,1.33,2,3$, 4, 7, 10 and 14. AA concentrations were determined by a validated stability-indicating liquid chromatographic method with UV detection. Chemical stability was calculated from the intersection of the lower limit of the $95 \%$ confidence interval of the observed degradation rate and the time to achieve $90 \%$ of the initial concentration.

Results: The analytical method separated degradation products from AA such that the concentration was measured specifically, accurately (deviations from known averaged $<2.2 \%$ ) and reproducibly (replicate error averaged $<1.82 \%(\mathrm{CV}[\%])$. During the study period at $4{ }^{\circ} \mathrm{C}$ all solutions retained more than $97.7 \%$ of the initial concentration and at $25^{\circ} \mathrm{C}$ more than $88 \%$ remained after 14 days, for both manufacturers. Study days and temperature significantly affected the percent remaining $(p<0.001)$ but solution $(p>0.495)$, concentration $(p>0.732)$ and manufacturer $(\mathrm{p}>0.808)$ had no significant effect.

Conclusions: AA concentrations between 36 and $92 \mathrm{mg} / \mathrm{mL}$, diluted in either NS or D5W and stored in PVC minibags are physically and chemically stable for at least 14 days at $4^{\circ} \mathrm{C}$ and 10 days at room temperature $\left(25^{\circ} \mathrm{C}\right)$ with PFL. Establishing a Beyond Use Date should be based on current NAPRA/USP-797 Guidelines.

\section{Stability of $2.5 \mathrm{mg} / \mathrm{mL}$ Indocyanine Green (ICG) Solutions Stored in Syringes at $25^{\circ} \mathrm{C}, 4^{\circ} \mathrm{C},-20^{\circ} \mathrm{C}$ and $-67^{\circ} \mathrm{C}$}

Perks $W^{1}$, Law $S^{1}$, Marchesano $R^{1}$, Walker $S E^{2}$

${ }^{1}$ Department of Pharmacy, Sunnybrook Health Sciences Centre, Toronto, ON ${ }^{2}$ Leslie Dan Faculty of Pharmacy, University of Toronto, Toronto, ON

Background: Due to increasing use of ICG, waste and recent backorders, chemical stability data was sought to preserve inventory.

Objective: To evaluate the chemical stability of ICG prepared in polypropylene (PP) syringes at concentrations of $2.5 \mathrm{mg} / \mathrm{mL}$ reconstituted with Sterile Water for Injection (SWFI) and stored at $25^{\circ} \mathrm{C}, 4^{\circ} \mathrm{C}$ and in the freezer $\left(-20^{\circ} \mathrm{C}\right.$ and $\left.-67^{\circ} \mathrm{C}\right)$ 
Methods: On study day 0, ICG solutions of $2.5 \mathrm{mg} / \mathrm{mL}$ were prepared in $5 \mathrm{~mL}$ PP syringes, reconstituted with SWFI. Three syringes were stored at $25^{\circ} \mathrm{C}, 4^{\circ} \mathrm{C},-20^{\circ} \mathrm{C}$ or $-67^{\circ} \mathrm{C}$. ICG concentrations were determined 8 times over each study period at each temperature using a validated stability indicating analytical method. Chemical stability was based on the intersection of the lower limit of the $95 \%$ confidence interval of the observed degradation rate and the time to achieve $90 \%$ of the initial concentration (T-90).

Results: The analytical method separated degradation products from ICG such that the concentration was measured specifically, accurately and reproducibly $(1.73 \%(\mathrm{CV}(\%))$. Analysis of variance revealed significant differences in percent remaining due to study day $(\mathrm{p}=0.009)$ and temperature $(\mathrm{p}=0.035)$. The calculated T-90, with $95 \%$ confidence, exceeded the 28-day study period for syringes stored in the freezer at either $-20^{\circ} \mathrm{C}$ or $-67^{\circ} \mathrm{C}$. The calculated T- 90 , with $95 \%$ confidence, was 34.11 hours at $25^{\circ} \mathrm{C}$ and 37.38 hours at $4^{\circ} \mathrm{C}$.

Conclusions: We conclude that $2.5 \mathrm{mg} / \mathrm{mL}$ solutions of ICG may be stored frozen at $-20^{\circ} \mathrm{C}$ or $-67^{\circ} \mathrm{C}$ for up to 28 days, but at $4^{\circ} \mathrm{C}$ or $25^{\circ} \mathrm{C}$, solutions should be stored for only 36 -hours. Syringes stored in the freezer for up 28-days can be withdrawn from the freezer, allowed to thaw, but should be used within 24-hours of withdrawal from the freezer. Under these conditions more than $92.5 \%$ of the initial ICG concentration will remain at 24 hours.

\section{Development of an On-Going Sterility Monitoring Program for Single-Use Vials Undergoing Multiple Access Following Application of a Closed System Transfer Device}

Charbonneau LF', Carating $H^{1}$, Mascioli $M^{1}$, Iazzetta $J^{1}$, Perks $W^{1}$, Stinson $J^{I}$, Nedzka-Rajwans $I^{1}$, Walker $S E^{2}$

${ }^{1}$ Department of Pharmacy Sunnybrook Health Sciences Centre, Toronto, ON ${ }^{2}$ Leslie Dan Faculty of Pharmacy, University of Toronto, Toronto, ON

Background: Closed system transfer devices (CSTD) are designed to protect healthcare workers from exposure to hazardous drugs. These devices have also been shown to minimize microbial contamination of single use vials (SUV). However, NAPRA has suggested that annual testing of the CSTD is necessary to assure continued sterility. Since validation requires more than 3000 transfers, the feasibility of an on-going monitoring program was investigated.

Objective: To test whether attaching a CSTD (Equashield®) to SUVs can minimize microbial contamination and extend the "use-by" date following multiple withdrawals under extreme-use-conditions.

Methods: An Equashield ${ }^{\circledR}$ vial adapter was attached to one 20 -mL vial containing sterile TSB growth medium and placed in each of 4 biological safety cabinets weekly. $1-\mathrm{mL}$ samples were drawn from each vial at immediately following application of the CSTD and at 48 and 168 hours. Prior to sample withdrawal vials were visually inspected for turbidity. After 1 week, vials were collected, incubated at $37 \mathrm{oC}$ for 14-days and inspected visually every 2 days for evidence of contamination (turbitidy). For positive controls, three TSB vials were inoculated with less than 102 of S.epidermidis ATC12228. As a negative control, three unopened vials of TSB were incubated for 14 days. Stopping rules included growth in 2 CSTD vials in fewer than 100 consecutive vials (contamination rate using lower limit of $80 \%$-Confidence Interval (CI) is greater than $0.20 \%$ ).
Results: All positive control vials demonstrated growth within 48-hours. All negative control vials showed no growth. During the first 20 -weeks of monitoring, all CSTD vials (80 vials - 240 transfers) remained sterile following storage at room temperature for 7 days and subsequent incubation for 14 -days. The $95 \%$-CI of the contamination rate is 0.000 to $0.021 \%$.

Conclusions: Attachment of a CSTD to single-use-vials within an ISO-5 environment has the ability to maintain sterility following multiple withdrawals over 7-days.

\section{Stability of $0.04,0.1$, and $0.2 \mathrm{mg} / \mathrm{mL}$ Vitamin $\mathrm{K}$ (Phytonadione) in 5\% Dextrose in Water Solutions Stored in Polyvinyl Chloride Bags at $4^{\circ} \mathrm{C}$ over 9 Days}

Plaetzer $W^{1}$, Facca $N^{2}$, Smith $N^{3}$

${ }^{1}$ Leslie Dan Faculty of Pharmacy, University of Toronto, Toronto, ON

${ }^{2}$ Department of Pharmacy, London Health Sciences Centre, London, ON

${ }^{3}$ Department of Pathology and Laboratory Medicine, 3London Health Sciences Centre, London, ON

Background: To our knowledge, no stability data exist for 0.04, 0.1 and $0.2 \mathrm{mg} / \mathrm{ml}$ intravenous vitamin $\mathrm{K}$ (phytonadione) solutions in $5 \%$ dextrose in water (D5W) in polyvinyl chloride (PVC) bags.

Objectives: To test the physical and chemical stability of the 0.04$0.2 \mathrm{mg} / \mathrm{ml}$ vitamin $\mathrm{K}$ solutions in D5W over 9 days at $4^{\circ} \mathrm{C}$.

Methods: Prepared solutions were stored at $4^{\circ} \mathrm{C}$ for 9 days, with samples taken daily and frozen pending analysis. Samples from selected days throughout the study were analysed in quintuplicate by gas chromatography/mass spectrometry, with vitamin K-d7 as the internal standard (ISTD). Vitamin K/ISTD peak area ratios (PARs) were calculated for and compared to those of Day 0 by calculating them as a percentage of the Day 0 value. Mass spectra of Day 9 drug peaks were compared to those of Day 0 peaks to confirm purity. Physical stability was assessed visually daily.

Results: The vitamin $\mathrm{K}$ concentration in the preparation of $0.04 \mathrm{mg} / \mathrm{ml}$ vitamin $\mathrm{K}$ in D5W declined, with the lower 95\% confidence limit falling below $90 \%$ of the Day 0 value within 45 hours. The lower limits of the $95 \%$ confidence intervals of the $0.1 \& 0.2 \mathrm{mg} / \mathrm{ml}$ vitamin $\mathrm{K}$ solutions always remained above $90 \%$ of the day 0 values. Day 9 mass spectra of all solutions were identical to those of Day 0 . All solutions remained visually consistent over the 9 days.

Conclusions: These data support $0.1 \& 0.2 \mathrm{mg} / \mathrm{ml}$ vitamin K solutions in D5W as physically and chemically stable for 9 days, but $0.04 \mathrm{mg} / \mathrm{ml}$ vitamin $\mathrm{K}$ in D5W was chemically stable for less than 2 days. 


\section{Élaboration d'outils et de politiques et procédures sur les préparations stériles}

Boyer $H^{1}$, Forest ${ }^{2}$, Guenette $S^{3}$, Lalonde $F^{4}$, Pelletier $D^{5}$, Richer $M^{6}$, Robillard $P$

${ }^{1}$ Département de pharmacie, Hôpital Anna-Laberge, Centre intégré de santé et de services sociaux de la Montérégie-Ouest, Châteauguay, QC

${ }^{2}$ Département de pharmacie, Centre hospitalier universitaire Sainte-Justine, Montréal, QC

${ }^{3}$ Département de pharmacie, Hôpital Notre-Dame, Centre intégré universitaire de santé et de services sociaux du Centre-Sud-de-l'Ille-deMontréal, Montréal, QC

${ }^{4}$ Association des pharmaciens des établissements de santé du Québec, Montréal, QC

${ }^{5}$ Département de pharmacie, Centre universitaire de santé McGill, Montréal, QC

${ }^{6}$ Département de pharmacie, Hôpital de la Cité-de-la-Santé, Centre intégré de santé et de services sociaux de Laval, Laval, QC

${ }^{7}$ Département de pharmacie, Hôpital Pierre-Boucher, Centre intégré de santé et de services sociaux de la Montérégie-Est, Longueuil, QC

Contexte : En 2014, l'Ordre des pharmaciens du Québec (OPQ) a publié les normes 2014.01 et 2014.02 sur la préparation de produits stériles non dangereux et non dangereux en pharmacie. Les départements de pharmacie ont l'obligation de répondre aux exigences de ces 2 normes, notamment en élaborant des politiques et procédures (P\&P) sur les différents aspects de la préparation de produits stériles.

Description : Soutenir les départements de pharmacie en proposant une série d'outils et de P\&P adaptés aux exigences de ces 2 normes.

Action : Sur la base des publications disponibles sur le sujet, élaborer des outils et P\&P requis par les 2 normes. S'assurer que les outils et P\&P soient adaptables aux réalités de tout type d'établissement.

Évaluation : Un groupe de travail composé de 7 pharmaciens pratiquant dans le domaine des préparations stériles a été créé avec pour mandat d'élaborer des outils et des P\&P. Avant d'être publiés, les outils et P\&P ont franchi les étapes suivantes : rédaction, validation en groupe, révision scientifique, validation des commentaires, révision linguistique et mise en page. Le groupe de travail a publié 103 outils et P\&P abordant les thèmes suivants : personnel et installations, préparations stériles et gestion de la qualité. Il a également publié : une foire aux questions, une boîte à outils et une procédure d'évaluation bisannuelle des compétences des pharmaciens désignés au soutien.

Répercussions : La possibilité d'utiliser ces outils et P\&P adaptables se traduit par une économie d'heures considérable pour les départements de pharmacie. Ils servent également de références pour l'évaluation et la formation du personnel assigné à ce secteur et à la standardisation des pratiques. Un sondage de satisfaction a révélé que $95 \%$ (142 sondés sur 149) des pharmaciens ayant utilisé les outils et P\&P les ont cotés 4 ou 5 (5 étant le maximum).
Stability of 9.1, 10.7 and $16.7 \mathrm{mg} / \mathrm{mL}$ Calcium Gluconate Solutions in Normal Saline and/or 5\% Dextrose in Water Stored in Polyvinyl Chloride Minibags at $4^{\circ} \mathrm{C}$ over 9 Days

Plaetzer $W^{1}$, Facca $N^{2}$, Smith $N^{3}$

${ }^{1}$ Leslie Dan Faculty of Pharmacy, University of Toronto, Toronto, ON

${ }^{2}$ Department of Pharmacy, London Health Sciences Centre, London, ON

${ }^{3}$ Department of Pathology and Laboratory Medicine, London Health

Sciences Centre, London, ON

Background: To our knowledge, no stability data exist for 9.1, 10.7 or $16.7 \mathrm{mg} / \mathrm{ml}$ calcium gluconate solutions in $5 \%$ dextrose in water (D5W) or normal saline solutions stored in polyvinyl chloride (PVC) bags at $4^{\circ} \mathrm{C}$.

Objective: To test the physical and chemical stability of the calcium gluconate solutions, in diluents D5W or normal saline, stored in PVC minibags at $4^{\circ} \mathrm{C}$ for 9 days.

Methods: The calcium gluconate solutions were stored in PVC bags at $4^{\circ} \mathrm{C}$ for 9 days. Samples were taken daily and frozen at $-80^{\circ} \mathrm{C}$, pending analysis. Samples from selected days were analysed in quintuplicate on a Roche Cobas C 701 analyzer. Calcium concentration means from each selected day were compared to those of Day 0 by calculating them as a percentage of the Day 0 value. Chemical stability was demonstrated if the lower limit of the $95 \%$ confidence interval did not fall below $90 \%$ of the Day 0 value. Physical stability was assessed by daily colour and clarity observation.

Results: The lower limits of the $95 \%$ confidence intervals of all of the solutions remained above $90 \%$ of the Day 0 concentration for the entire 9 day study. All of the calcium gluconate solutions remained clear and colourless throughout the study.

Conclusion: These data support the assignment of a beyond-use date of 9 days for preparations of 9.1, 10.7 and $16.7 \mathrm{mg} / \mathrm{ml}$ calcium gluconate with diluents of either normal saline or D5W in PVC bags at $4^{\circ} \mathrm{C}$. 